\title{
YArat IsthND
}

Irvarion of Drow cowhars

B. H, hupbers

$15701,15702,15703,157031$ and 15729

15704,15705 and 15706

15707

15708

15709 and 15710

25711 and 25712

15713

15715

25717 and 15718

15719 and 15720

15721

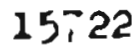

15723

15?26́

15724

$15 ? 25$

15726

15727 and 15728
Co-Ordinates plevetion

1400N; 800s 920 seet

1400N; $1000 \mathrm{r} 830 \mathrm{n}$

1400N; $1200 \mathrm{E} 750$ "

1200N; 12008770 "

$1000 \mathrm{~N} ; 1200 \mathrm{~B} \quad 830 \%$

1400N, 14008 700 "

1200N; $1400 \mathrm{R} 720$ "

$800 \mathrm{~N} ; 1400 \mathrm{R} 800$ "

$600 N ; 1400 \mathrm{x} 830$.

$1400 \mathrm{~N} ; 16008680$ "

1200n; 1600 s 700 "

1000N, 1600 I $720 "$

800N; $1600 \mathrm{R} 750$ "

200N; 200E 1610 n

200\%; $\infty 001550 "$

$\infty ; 00.1560$ "

2003; $\infty 1520$ "

$\infty ; 13581560 "$ 
Wht of Abbroviations 2 in In Yatobl Ioland for

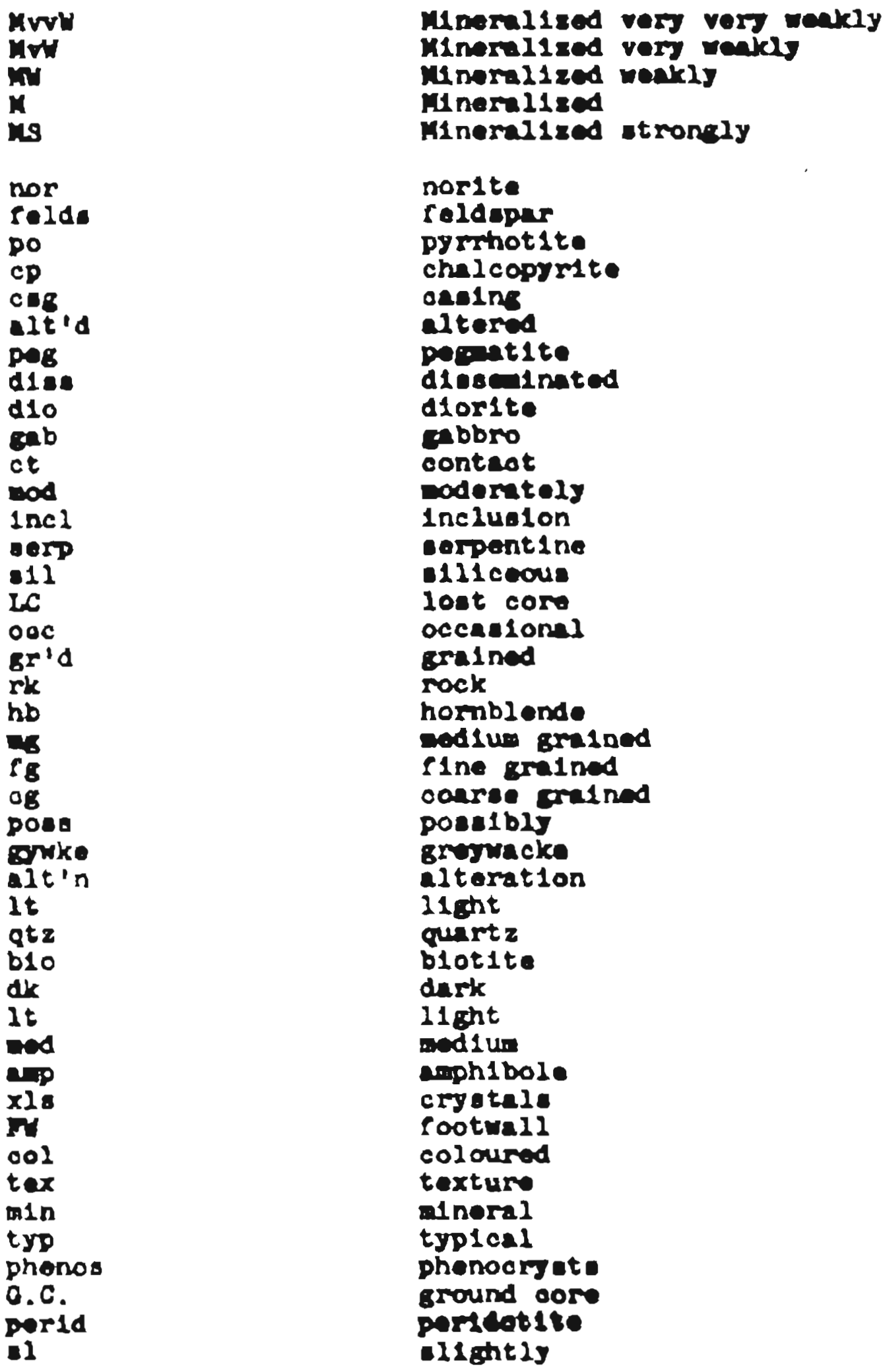




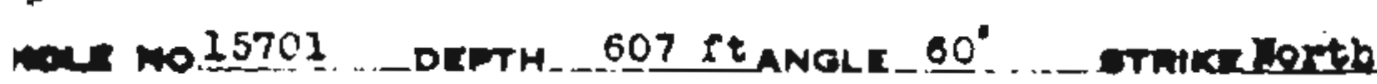

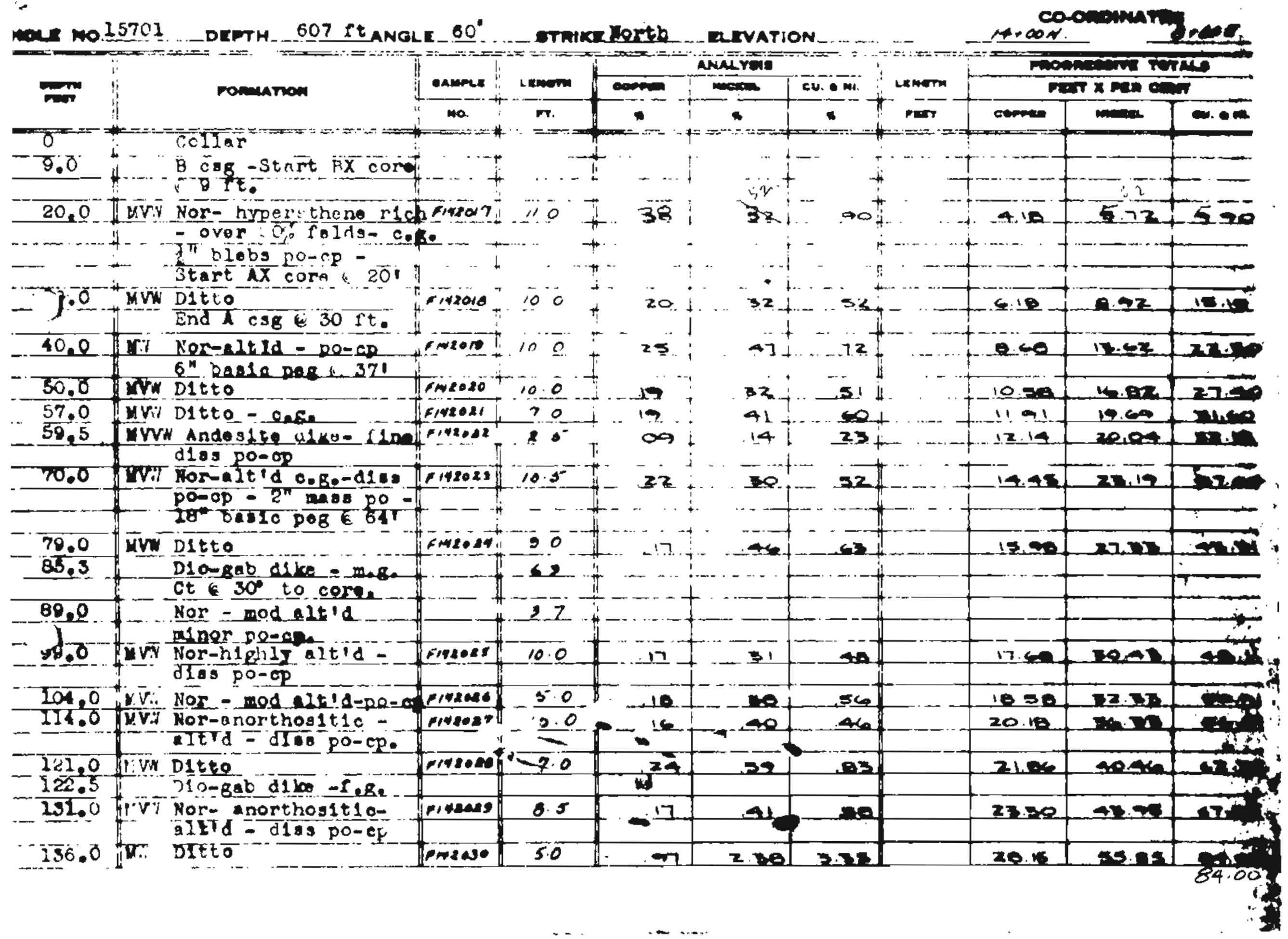


xx No.20rol

DDTH 607 ANGK $60^{\circ}$

omike Noth _arvation coOndinatra

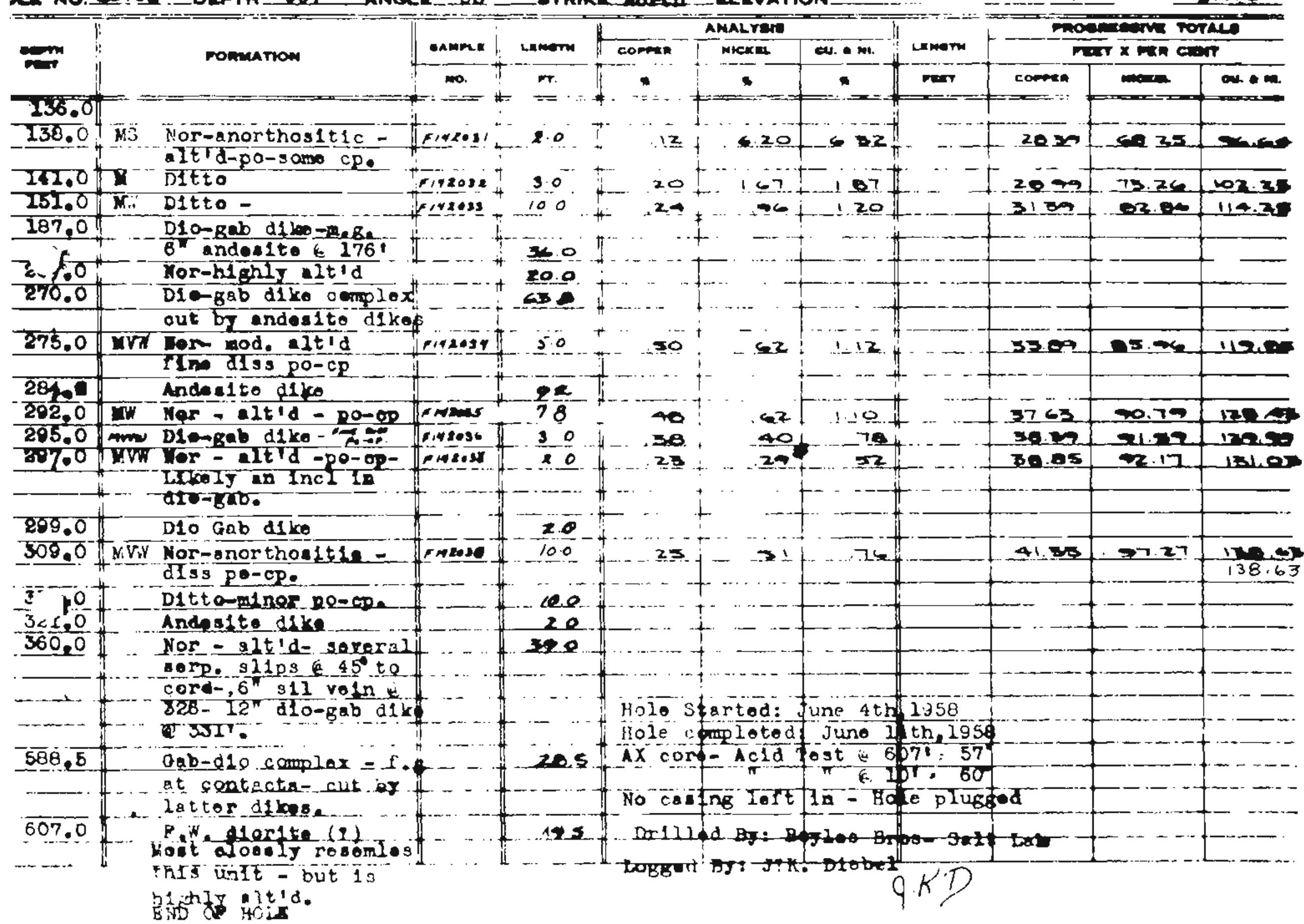




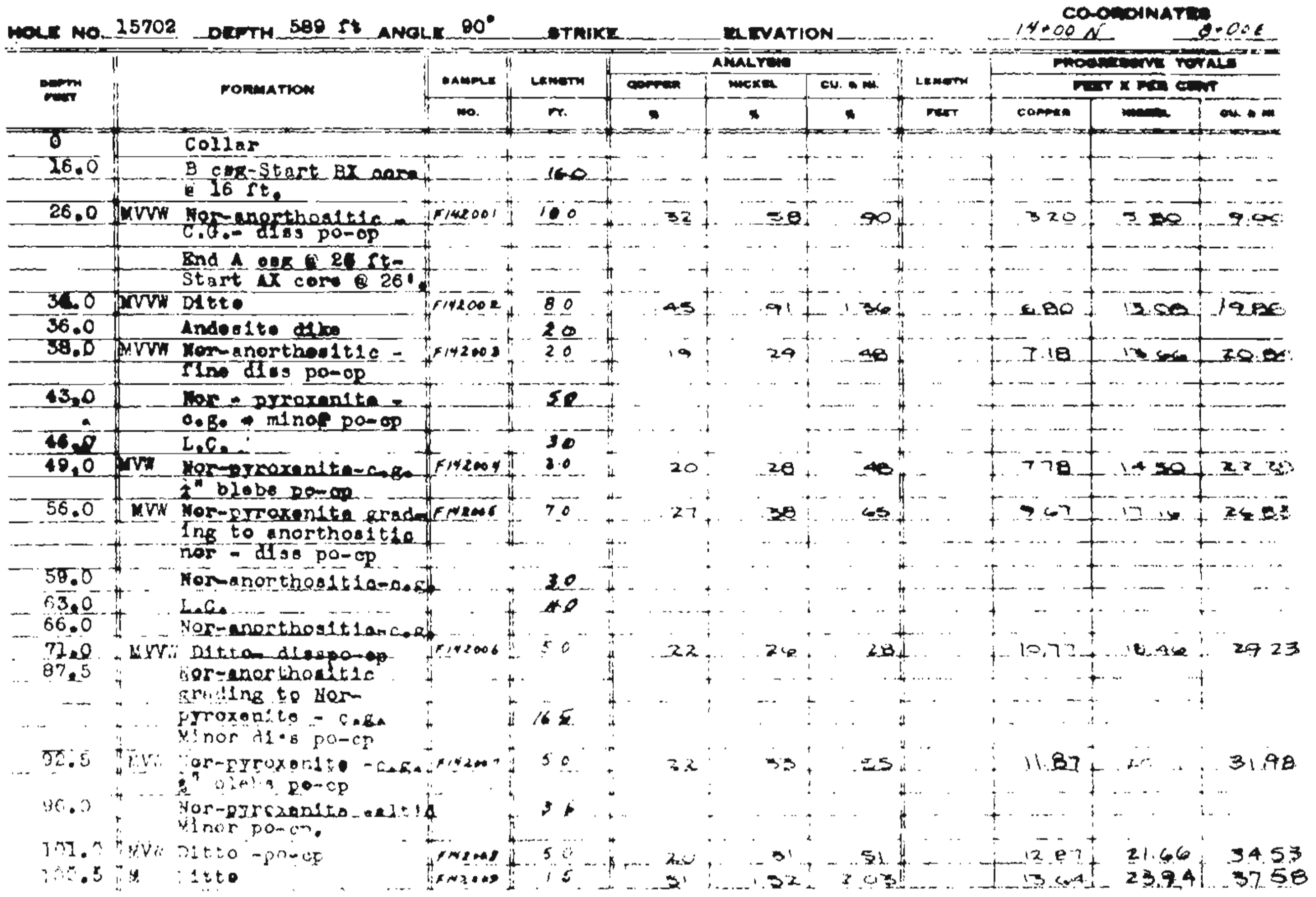




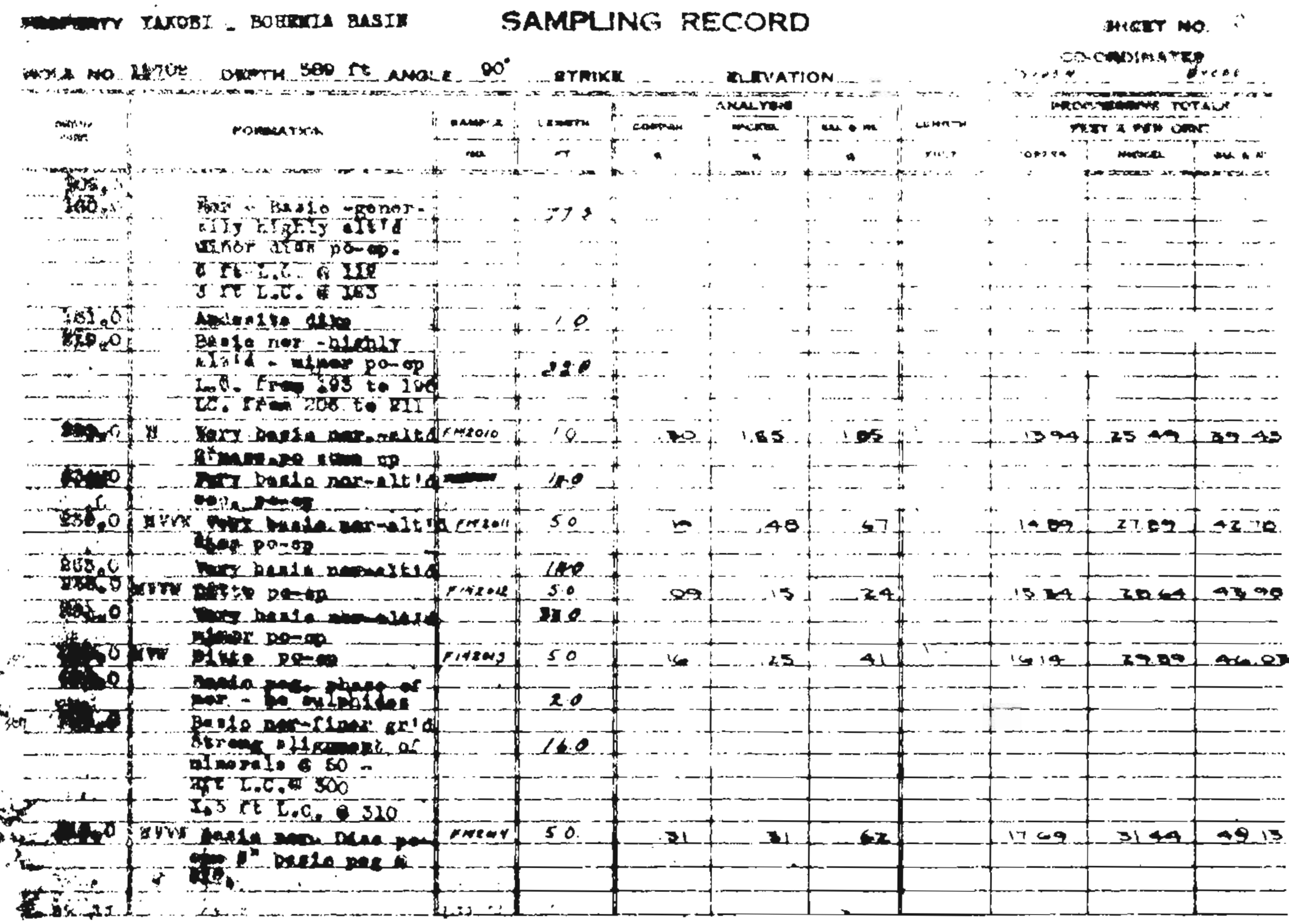

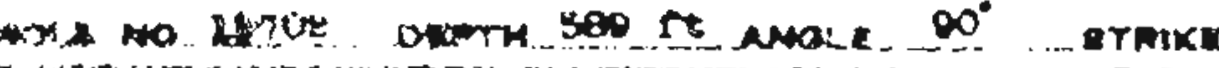

incom notise

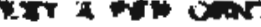

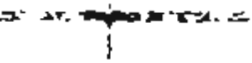

Axin 1 te aty.

$F=-200$ to

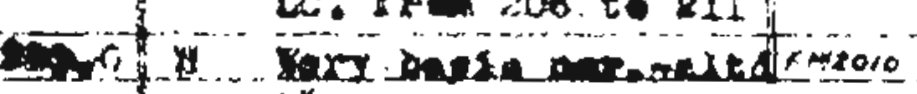

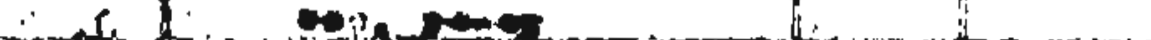

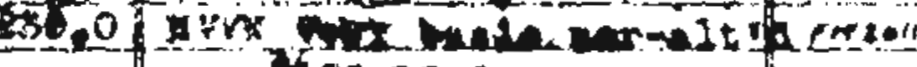
Q6o $c$ i

is here wim nux

mation

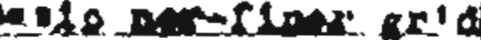

reac 418 and or

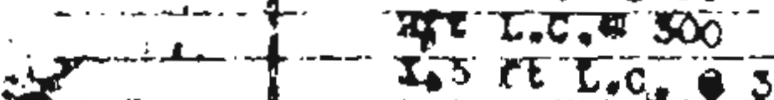

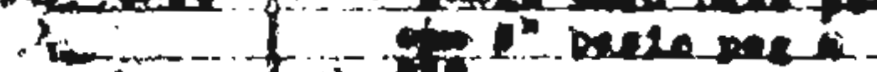

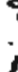




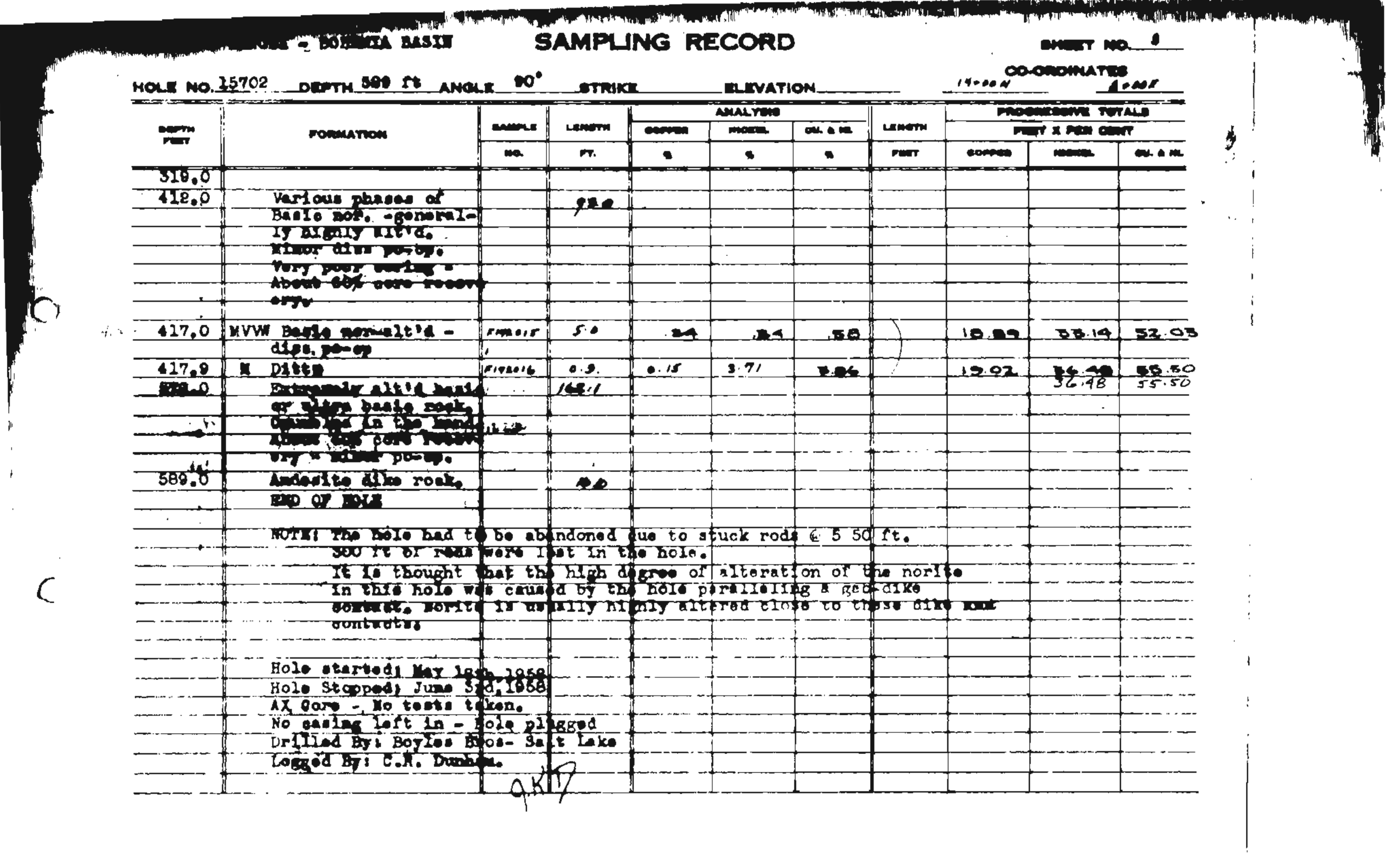




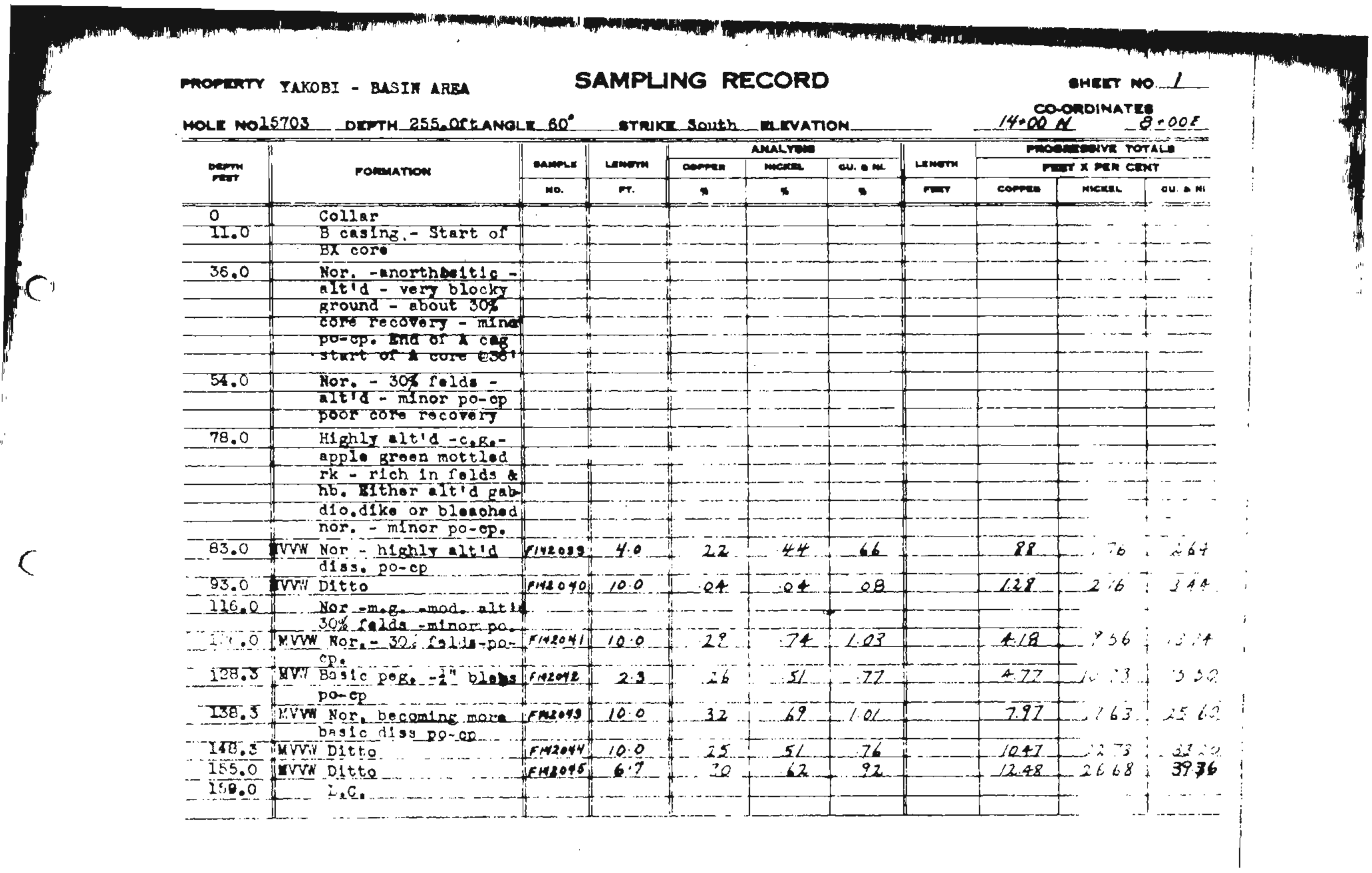




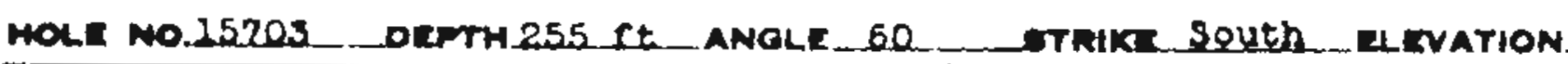

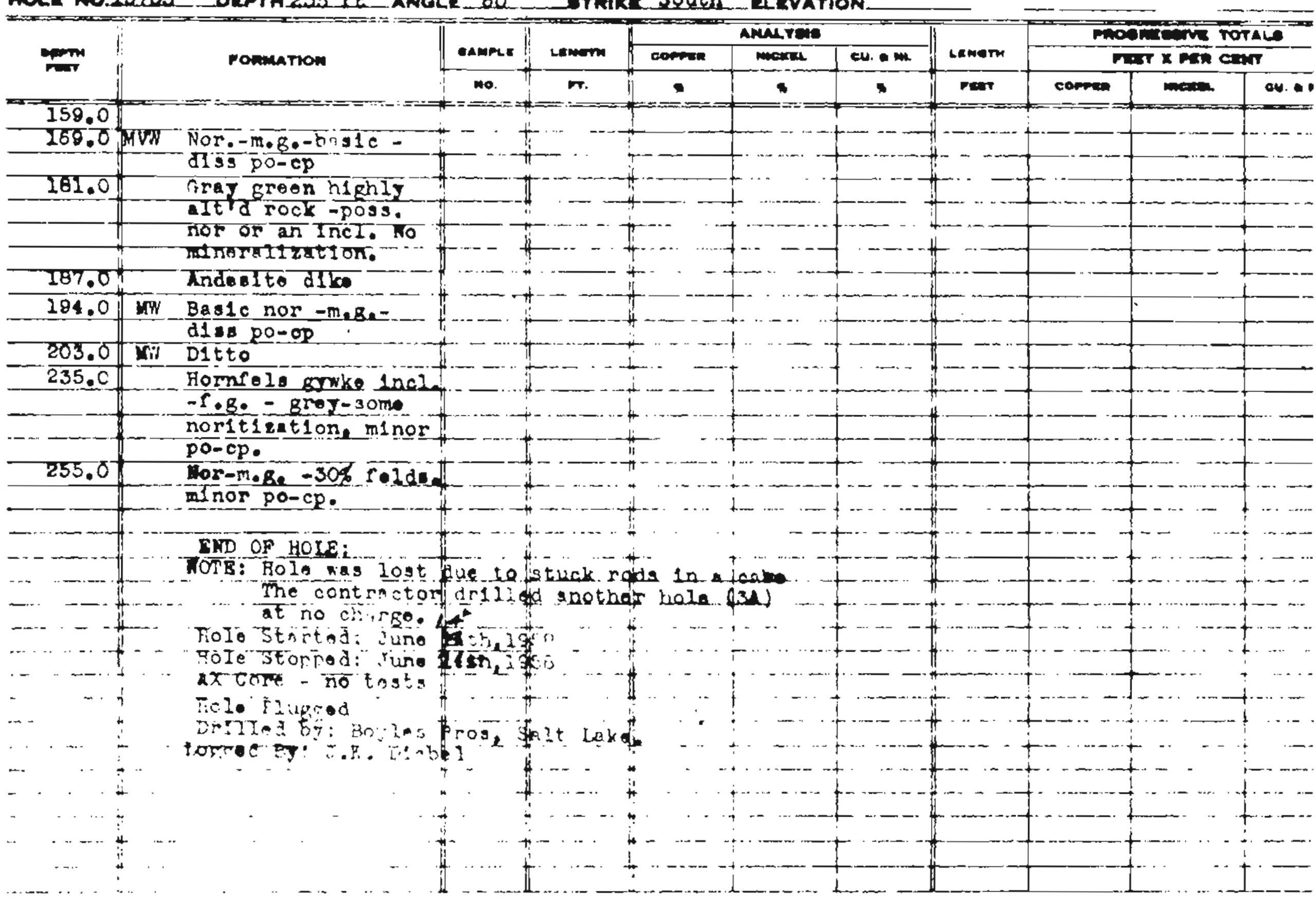




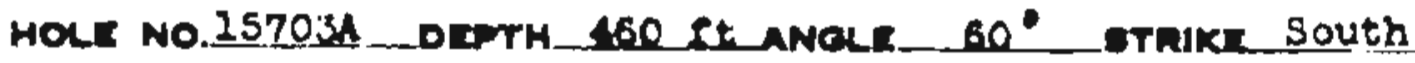

Havation

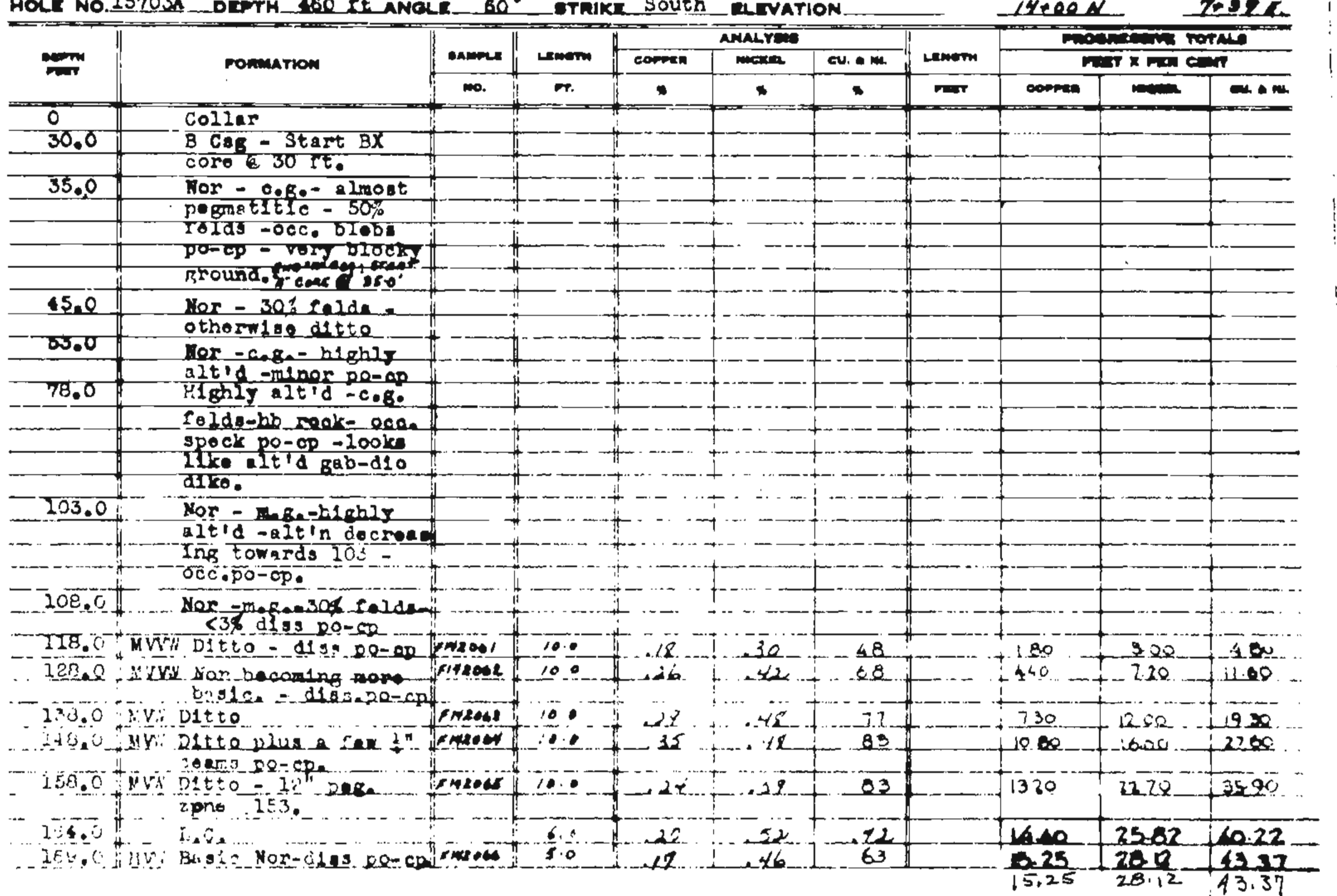


HOLE NO. 157034 _OEPTH . 500 ft ANGLE $60^{\circ}$ _etrake SOUth, ELVATION

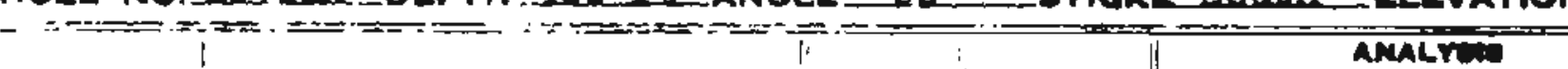
$14.004 \quad-7+27 c$ $\operatorname{atrm}$

ponmation

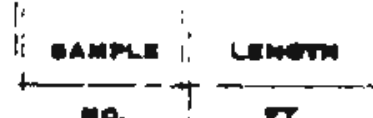
oormat.

169.01 no. $\pi$.

\begin{tabular}{|c|c|c|}
\hline mexth & c4 & Lmoth \\
\hline t & 1 & $m$ \\
\hline
\end{tabular}

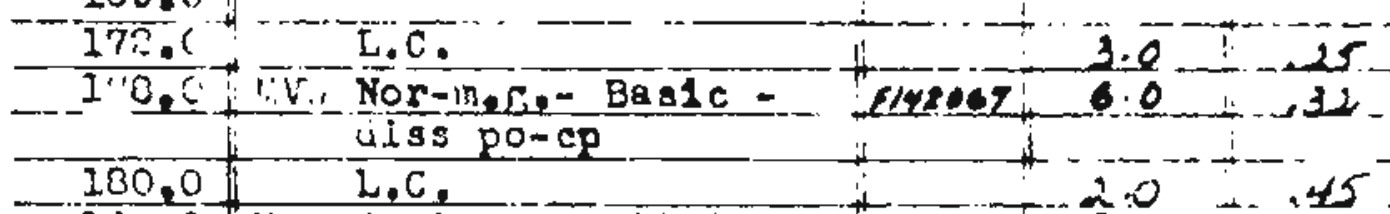

$$
\mathrm{L} . \mathrm{C} .
$$

$-$

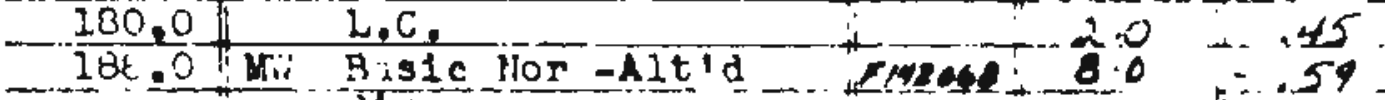
diss po-op

-195.0 Andeste dike - t $-10-$

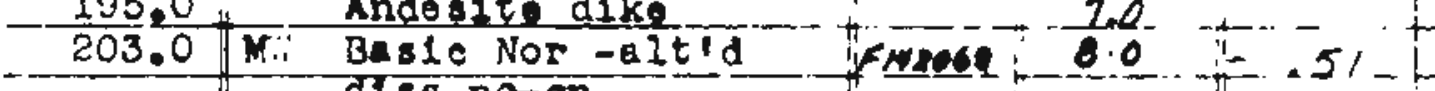

diss po-cp.

S.B.-Eroy-nortitzed.

- 1 reer contrcts - occ.j

249.5 D1o-gab dike $-5 \mathrm{~g}$

$260.0]$

-270.0 mor or po-cp

blebs po-cp Ln to

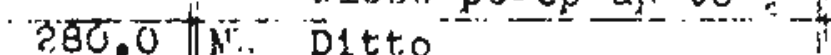

$235,0+\cdots, 010$

$\therefore 35,0$ my lor-mo-50.

"ome ztandsis $50-c^{4}$

297.6

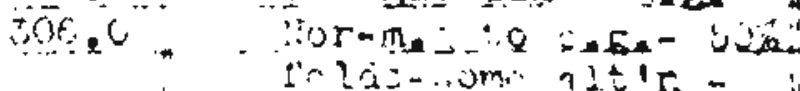

relno:

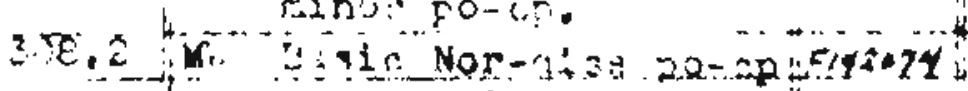
vy not 18 eqde

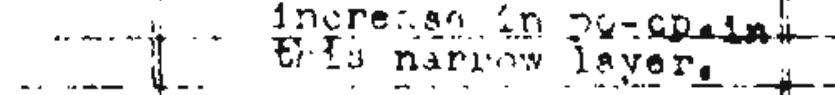

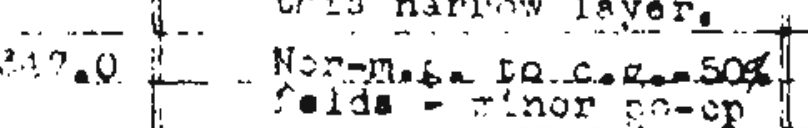

10.00

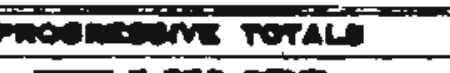
and an

comes mekri

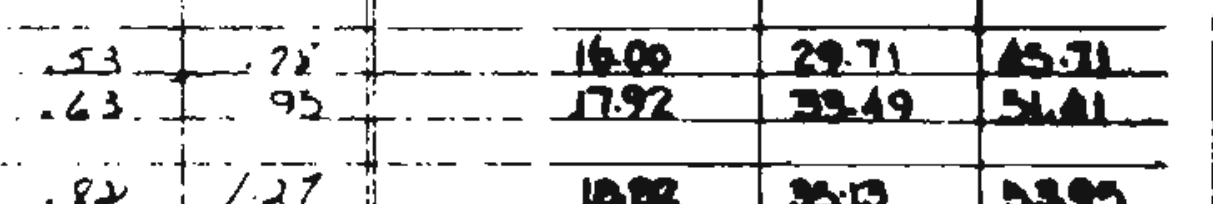

. $81 .+17$

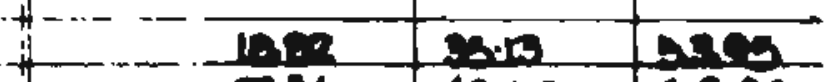

it$$
\text { (1) }
$$

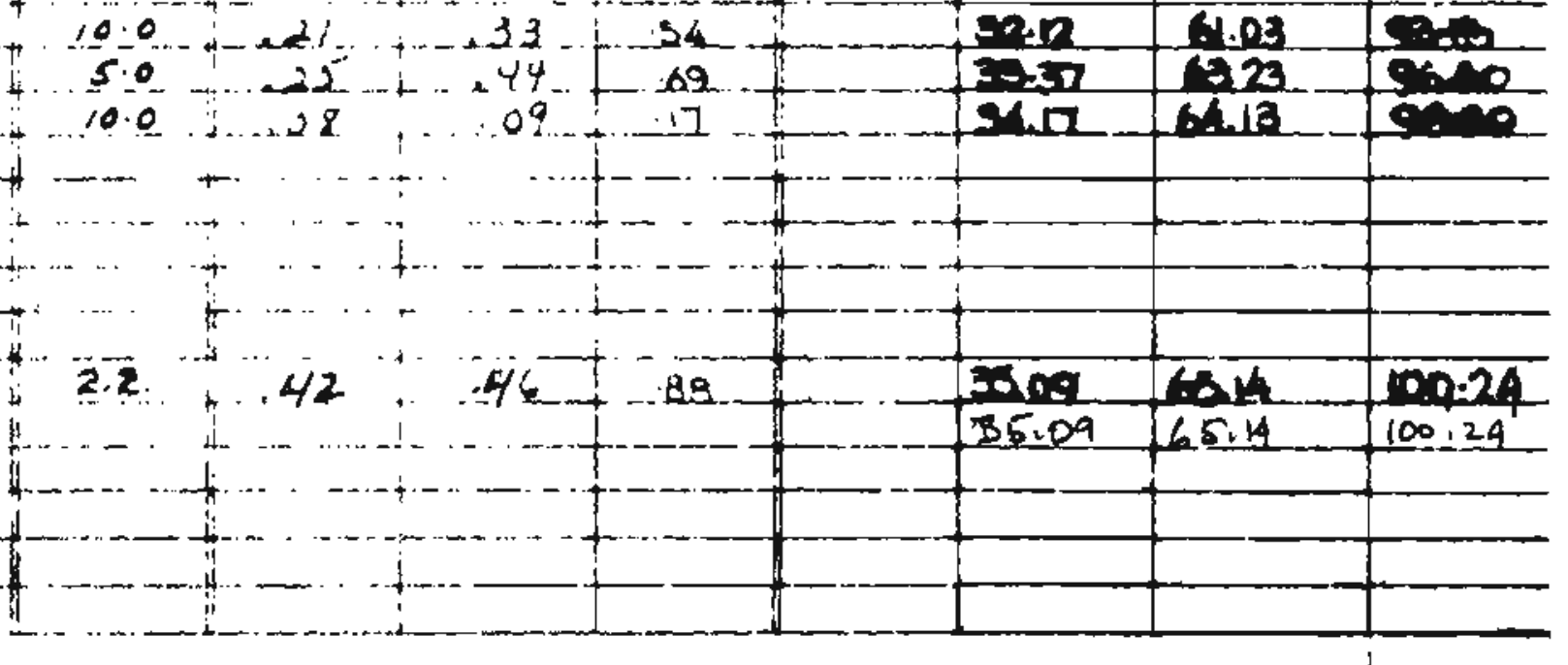




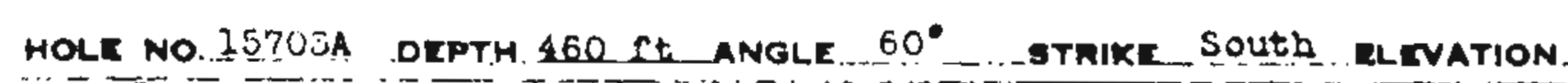

OHAT NO:'

$=-==-1=$

$\operatorname{lom}$

ronmation

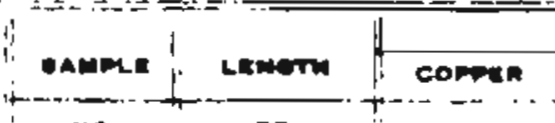
AnALrate comolnatte

347.0 no. $1+\infty$

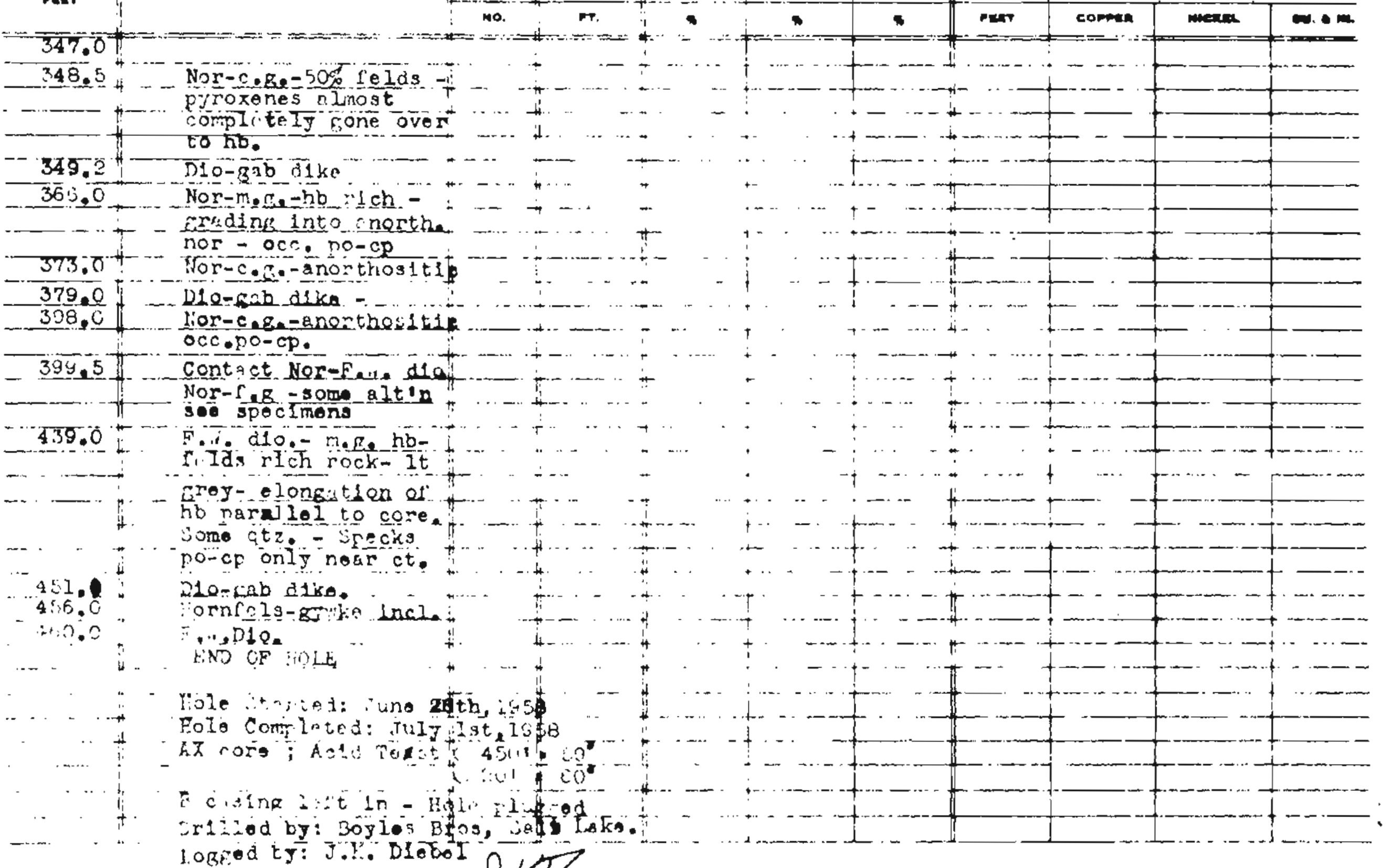
loged ty: J... Dlobol O.K.T. 


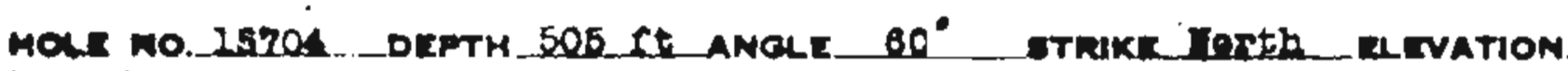

\begin{tabular}{|c|c|c|c|c|c|c|c|c|c|c|}
\hline \multirow{3}{*}{ nim } & \multirow{3}{*}{ Ponvation } & \multirow{3}{*}{$\frac{\text { encour }}{\text { ma }}$} & \multirow{3}{*}{$\frac{\text { crmon }}{\pi .}$} & \multicolumn{3}{|c|}{ Anarrote } & \multirow{3}{*}{$\frac{\operatorname{Lrmm}}{\operatorname{mat}}$} & \multirow{2}{*}{\multicolumn{3}{|c|}{ 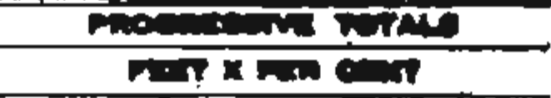 }} \\
\hline & & & & \multirow{2}{*}{$\frac{\infty}{n}$} & \multirow{2}{*}{$\operatorname{man}$} & \multirow{2}{*}{ 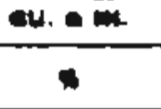 } & & & & \\
\hline & & & & & & & & commat & mats & $\because 0$ \\
\hline 0 & \multicolumn{10}{|l|}{$\cos 1 \mathrm{~s}$} \\
\hline \multirow{2}{*}{\multicolumn{11}{|c|}{$8 \operatorname{cog}=$ Stant $\mathrm{BX}$ 005. }} \\
\hline & & & & & & & & & & \\
\hline \multirow{2}{*}{\multicolumn{11}{|c|}{ arb-dio diko }} \\
\hline & & & & & & & & & & \\
\hline \multicolumn{11}{|c|}{$0+20 \Gamma E-\operatorname{sen} x$} \\
\hline \multirow{2}{*}{\multicolumn{11}{|c|}{$a+b-d 10$}} \\
\hline & & & & & & & & & & \\
\hline \multirow{3}{*}{\multicolumn{11}{|c|}{111.0}} \\
\hline & & & & & & & & & & \\
\hline \multirow{2}{*}{\multicolumn{11}{|c|}{ Jornigh elt'd - }} \\
\hline \multirow{2}{*}{\multicolumn{11}{|c|}{ 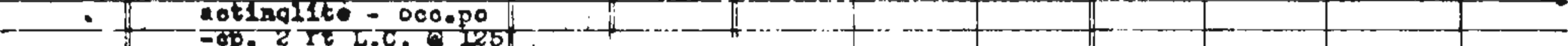 }} \\
\hline & & & & & & & & & & \\
\hline \multirow{2}{*}{\multicolumn{11}{|c|}{$-\overline{157.0}$}} \\
\hline & & & & & & & & & & \\
\hline \multirow{2}{*}{\multicolumn{11}{|c|}{152,0}} \\
\hline & & & & & & & & & & \\
\hline \multirow{2}{*}{\multicolumn{2}{|c|}{ Vh Nor-blebs po- co un }} & & & - & (2. & & & & & \\
\hline & SVh Nor-blebs pow op up. & $1 * 2+1$ & $\angle 00$ & 29 & 42 & בt & & 2.20 & 4.20 & x.10 \\
\hline & te & & & ... & & & & & & \\
\hline \multicolumn{11}{|c|}{-236.0} \\
\hline \multicolumn{11}{|c|}{$--\quad-\quad-\cdots$} \\
\hline \multirow{2}{*}{\multicolumn{11}{|c|}{$\begin{array}{l}239.0 \\
300.0\end{array}$}} \\
\hline & colds & & & & & & & & & \\
\hline & $\cdots$ & & & -_ & & & & & & \\
\hline & ab-d1e & & 40 & & & & & & & \\
\hline & & & & & & & & & & \\
\hline$-2=-\frac{0}{61}$ & Lrit Nore- $\frac{1}{4}$ bletgs oo-op & {$[\because 2202+1$} & 75 & $\ldots+\ldots$ &.--15 & -12 & & $=40$ & 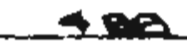 & $=27$ \\
\hline$\frac{3.36 .0}{34.0}$ & D10-gab dike - faga & & & & - & & & & & \\
\hline 312,0 & WV Nor-altd - t" blaba & $6 \times 2203$ & 6.0 & -+27 & 32 & $=0$ & & $\$ .0 x$ & $4=0$ & 1.9 .1 \\
\hline & $P_{-}-\mathrm{Ep}$ & & & & & & & 5.02 & & \\
\hline
\end{tabular}




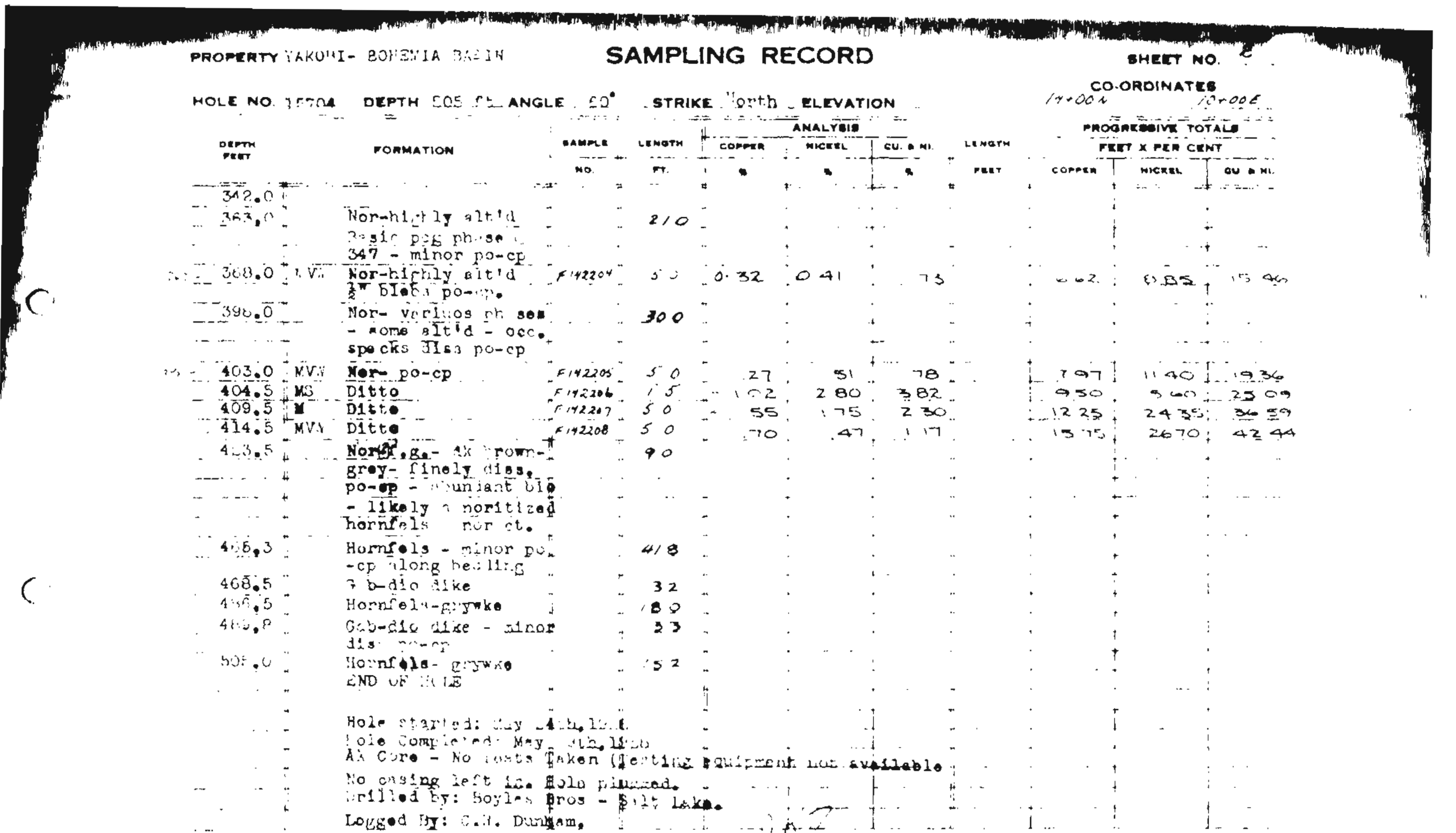




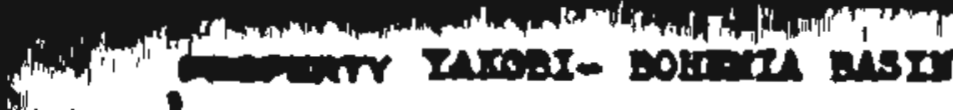

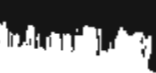

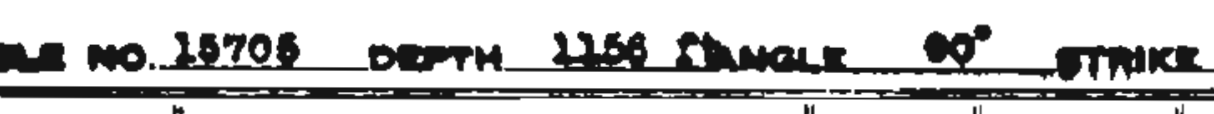

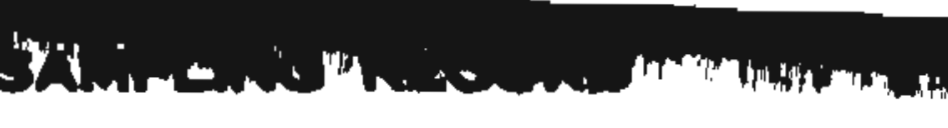

\begin{tabular}{|c|c|c|c|c|c|c|c|c|c|c|c|}
\hline & 10 & Dorm 2168 1 & o & & & Ext & & & $14 \infty$ & - & beve \\
\hline & & & & & & $\operatorname{anchich}$ & & & $\pi$ & 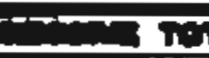 & rng \\
\hline$=$ & & nomentrom & anes & Lmam & $\cos$ & $=\infty$ & $\operatorname{lan}$ & Lemom & & $9 \times \operatorname{mos}$ & \\
\hline & & & $m$ & n. & 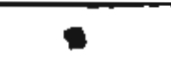 & - & $\bar{t}$ & mat & comenn & $\operatorname{mon}$ & $m \cdot m$ \\
\hline 366.0 & & & & & & & & & & & \\
\hline 380,0 & m & Ding nor $-310 \mathrm{chl} x$ & malos & 5.0 & .25 & .26 & $=1$ & & 4.78 & 6.50 & 11.05 \\
\hline & & up to t & & & & & & & & & \\
\hline 365.0 & & It coman. & & 6.9 & & & & & & & \\
\hline & & 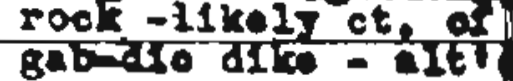 & & & & & & & & & \\
\hline 450.2 & & gab-d10 dilo - no & & $A+2$ & & & & & & & \\
\hline & & 1nol troin 442 to 44 & & & & & & & & & \\
\hline 45.0 & & Sele bleck rook = & & Ae & & & & & & & \\
\hline & & guite ol1, 11kolr a & & & & & & & & & \\
\hline Nepe & & $=-14 t+1=01+0$ & & $\rightarrow$ & & & & & & & \\
\hline 67 & & Torrotalled 1ned & & & & & & & & & \\
\hline & & or lele nortiondte & & & & & & & & & \\
\hline 5024 & & Ior pod $1 t^{\prime} a-900$ & & 224 & & & & & & & \\
\hline & & gock po-gp & & & & & & & & & \\
\hline 506.4 & swi & Emalded $-t^{\prime \prime}$ blobl & Evew & r.ㅇ. & .2s &.- .63 & $1 . \infty$ & $\dot{-}$ & 600 & $2=3$ & 16.95 \\
\hline & & $p=\operatorname{sen} \sigma$ & & & & & & & & & \\
\hline$\frac{516.0}{526.0}$ & In & ditte & $2 \operatorname{mens}$ & 9.6 & 31 & 23 & 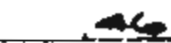 & & $0 .=2$ & 4.95 & 20.47 \\
\hline 826,0 & in & Dor d158.00000 & 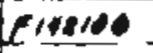 & 100 & 20 & 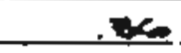 & 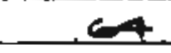 & & 11.32 & $1=3.5$ & 2697 \\
\hline 586,0 & inis & Diten & citare? & ne. & 30. & 2,2 & +42 & & 13.32 & 17.75 & .01 .07 \\
\hline 546,0 & ant & D1tta & $F+100$ & 10.0 & 호 & - se & 90 & & 14.28 & 2325 & -300 \\
\hline$\frac{569}{50}=$ & 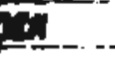 & Dete & r,anes & 60 & $-x$ & $-1-3$ & $\infty$ & 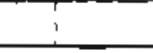 & 10.90 & 2그으으 & $-\infty 01$ \\
\hline 562.0 & & Ereoce spocks pa & & 90 & & $-2 \pi$ & & & & & \\
\hline 564,9 & & Andecite dike & & 3.2 & & & & & & & L- \\
\hline 572,0 & & $03 b-d 10$ d1kn & & 71 & $\ldots$ & $\ldots$ & & & & & -- \\
\hline 577 & Inw. & Contas ohsen battro & Finz"no. & 5.0 & -18 & $\therefore$ & 35 & & 19.00 & $2 A B$ & as 74 \\
\hline$\cdots$ & & geb-dle k me & $\ldots$ & & & & & & & & $-\ldots$ \\
\hline 500.2 & & Basto nor-ose & & 22 & & & & & & & 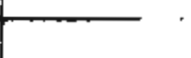 \\
\hline & & & & & & & & & & & \\
\hline & & & & & & & & & & & \\
\hline
\end{tabular}


HOLF NO. 15705 _DLPTH 1156 ft ANGLE $90^{\circ}$ _ETRIKK

\begin{tabular}{|c|c|c|c|c|c|c|}
\hline \multicolumn{4}{|c|}{ MLNATION } & \multicolumn{3}{|c|}{$1+\infty+\infty N$ - } \\
\hline \multicolumn{3}{|c|}{ AMALYOE } & \multirow[b]{2}{*}{ Lenom } & \multirow{2}{*}{\multicolumn{3}{|c|}{ montim rotale }} \\
\hline coments & mentes & cu. $\bullet \mathbf{m}$. & & & & \\
\hline פ & $s$ & • & mat & connen & $\operatorname{mos}$ & ov. $\mathrm{NI}_{1}$ \\
\hline
\end{tabular}

589.2

Fonkation

\begin{tabular}{|c|c|}
\hline oamme & LEMOTH \\
\hline & $\pi$. \\
\hline
\end{tabular}

$604, \frac{5}{2}+1$

Besic nor-pyroxented ent"m in blobs po sone cpo

6090 in o1t

617,0

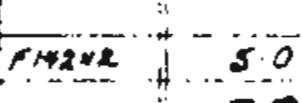
Banle nor. 620 il Bande nor

627.9 MW

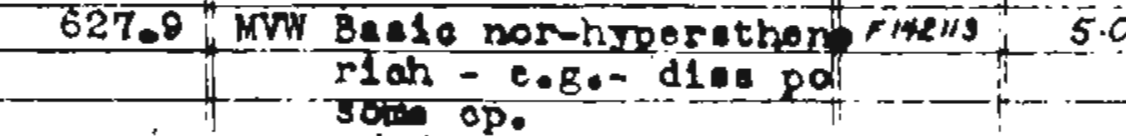

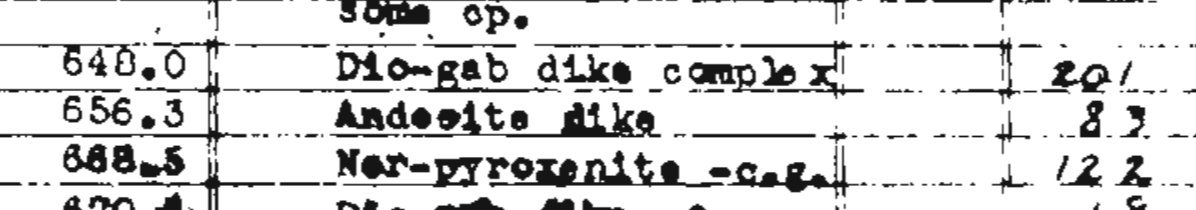

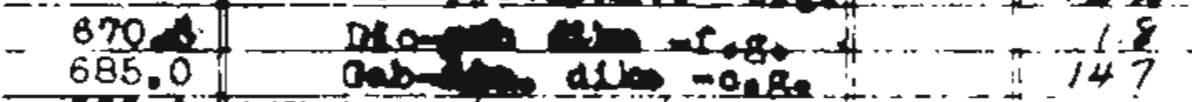

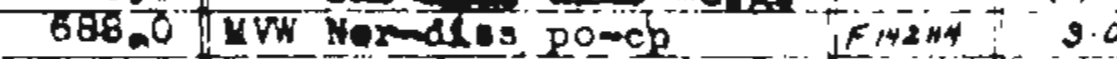

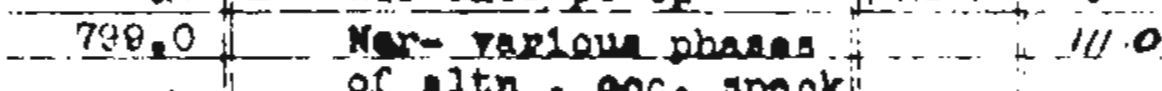

.. of Itn ooce spook

_... . . po-ap- Note 1t. bro

$-\cdots \cdots=$ hipera

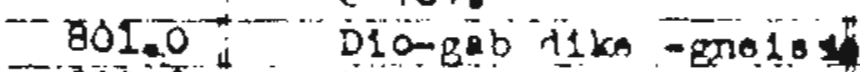

Nor - i. - it

- ... i - brom = Itd occ...

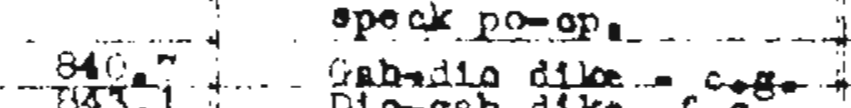

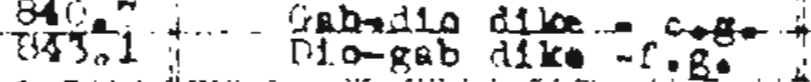

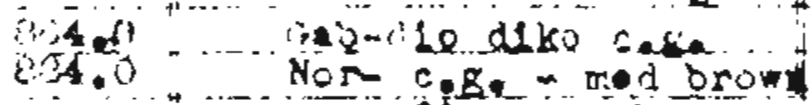

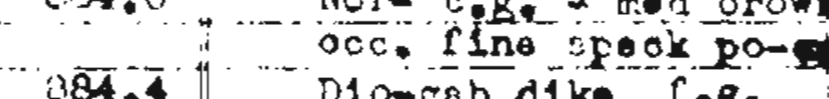

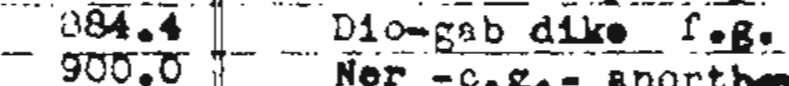

900.0 Nor-c.g-anorthent
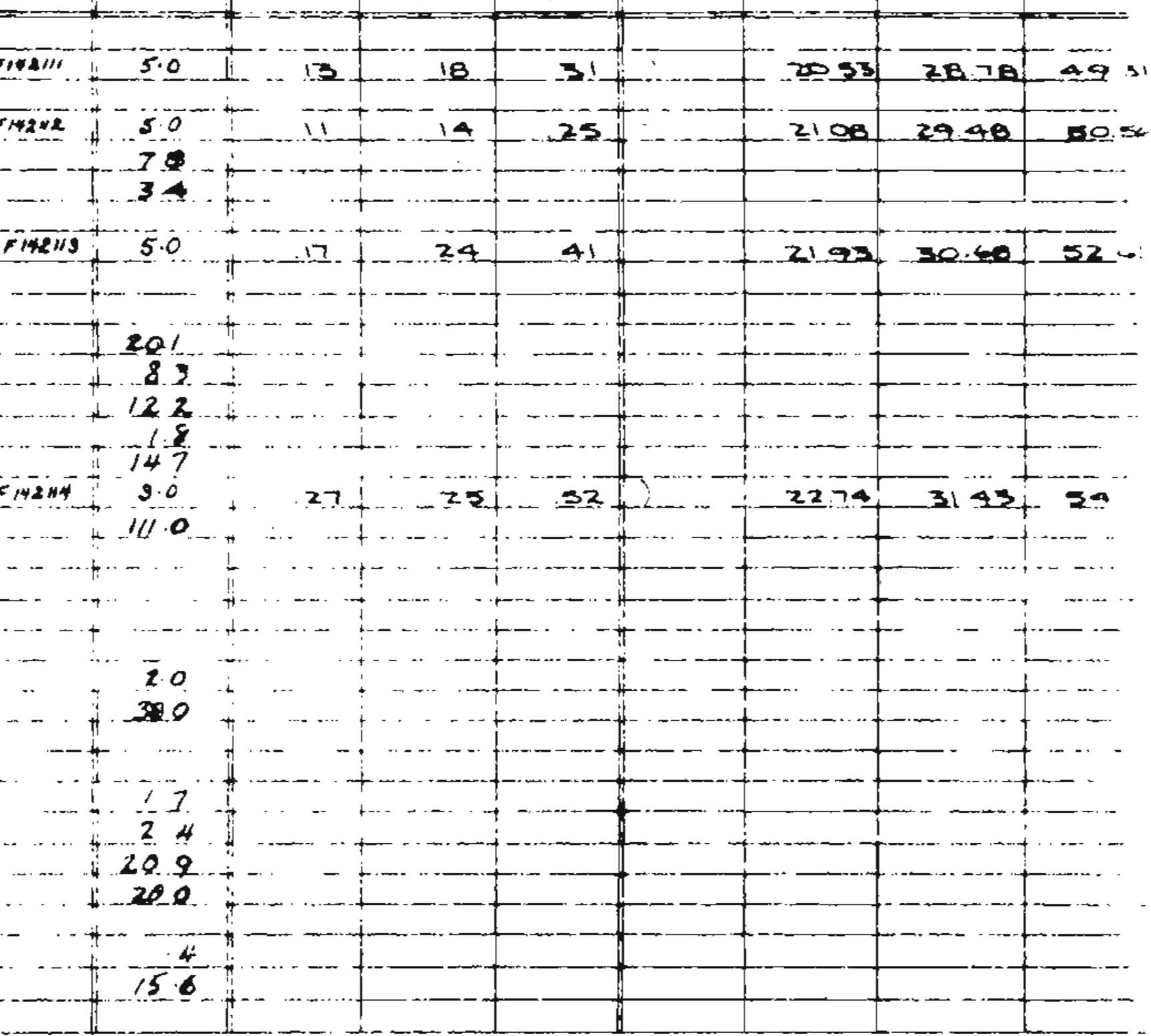


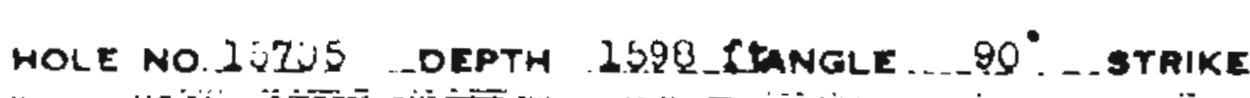
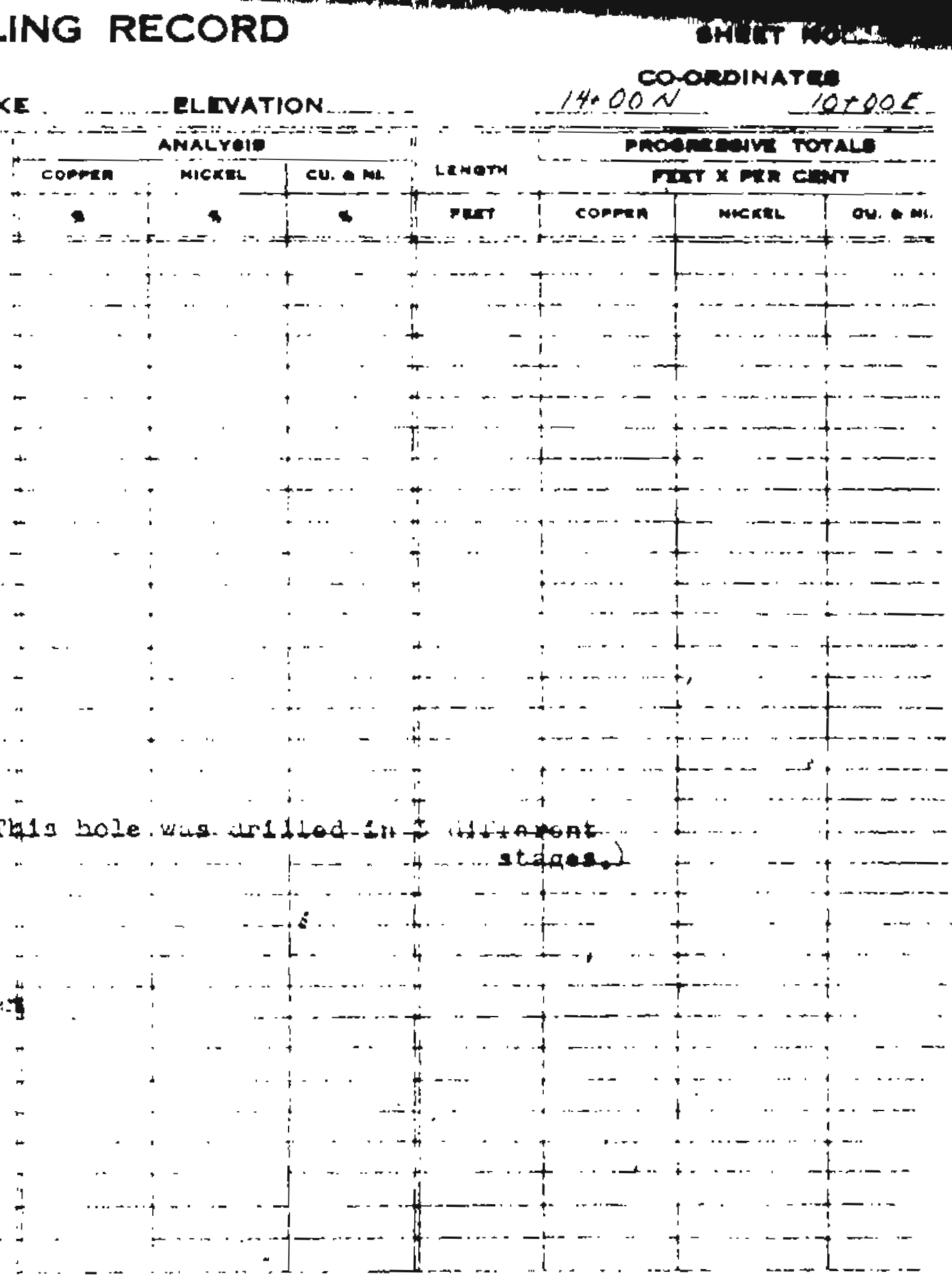

$$
\operatorname{Dem}
$$

FonMATION

inumer

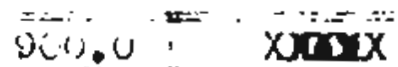

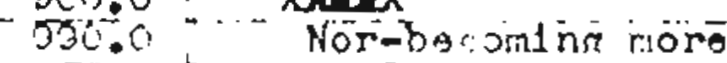

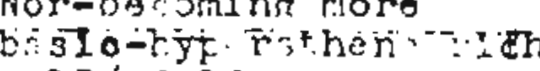

- $15 \%$ r. $31 \%$

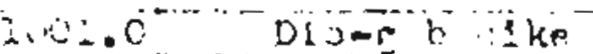

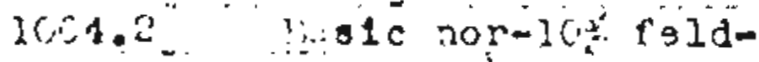

- oco "nrek no-cp

1605.5 210-t:-b.lice

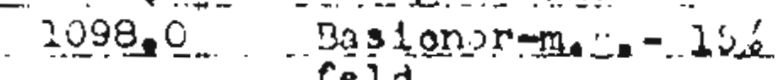

- - $\quad-$ feld.

JWar. - llowab ilko

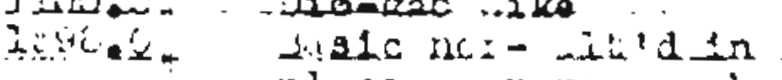

nlace. - riro ack

po-cp-v. ry o: i, ilo

Fib alka idp to $36^{n}$

GND OP "IICLE.

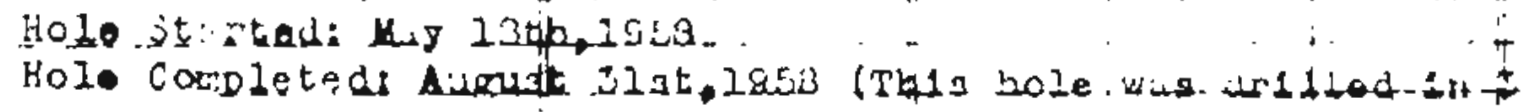

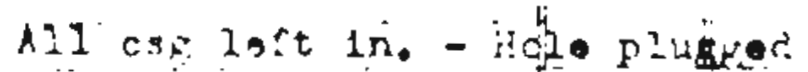

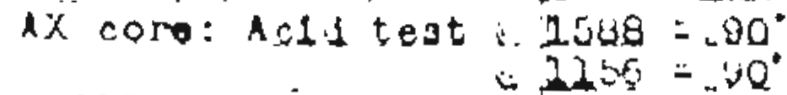

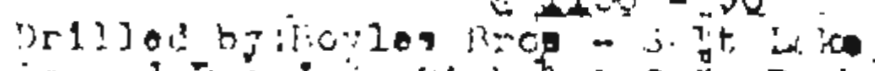

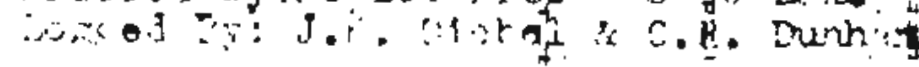




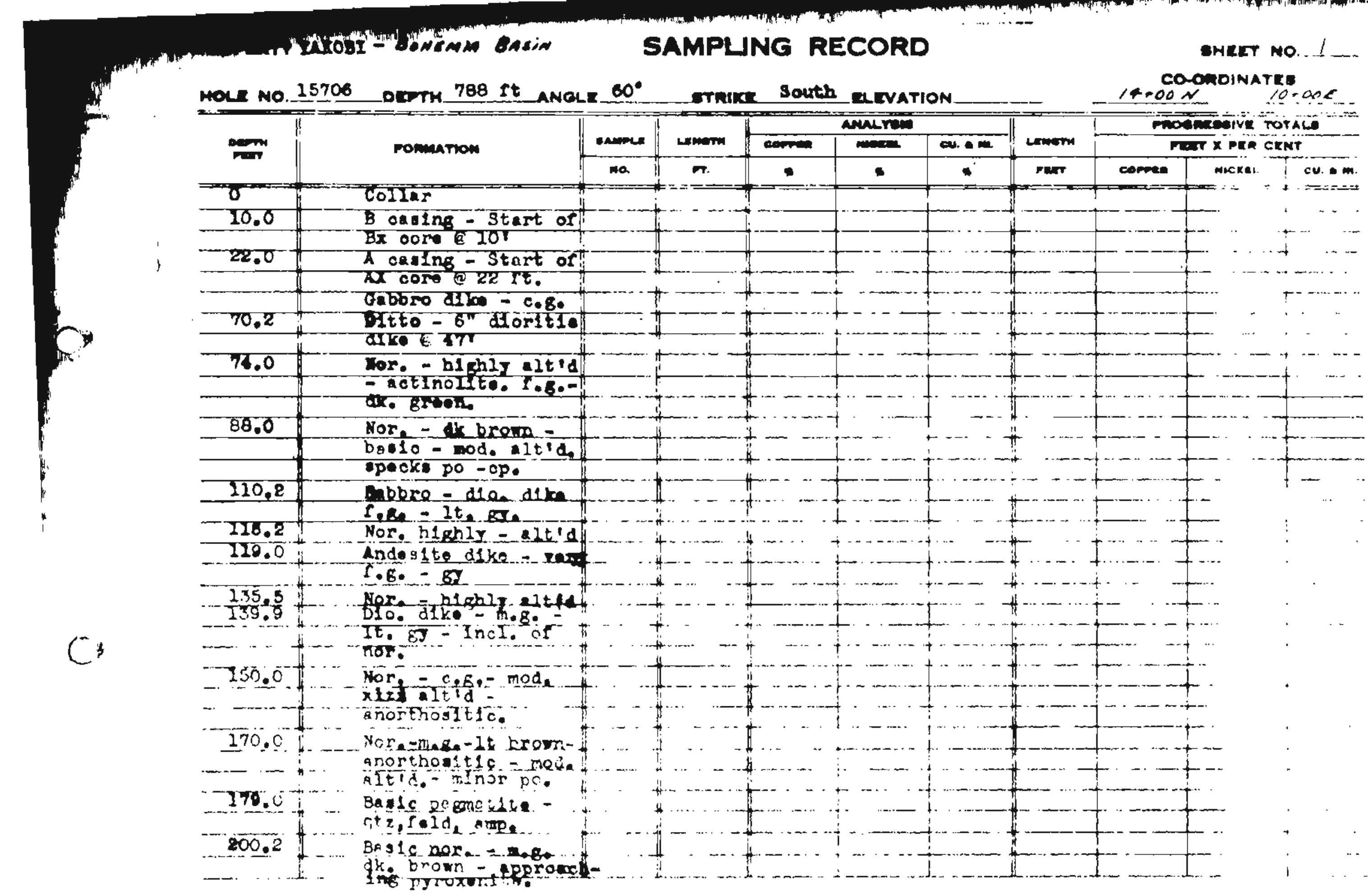


DTPH 7801 ANGL $60^{\circ}$

Truke South

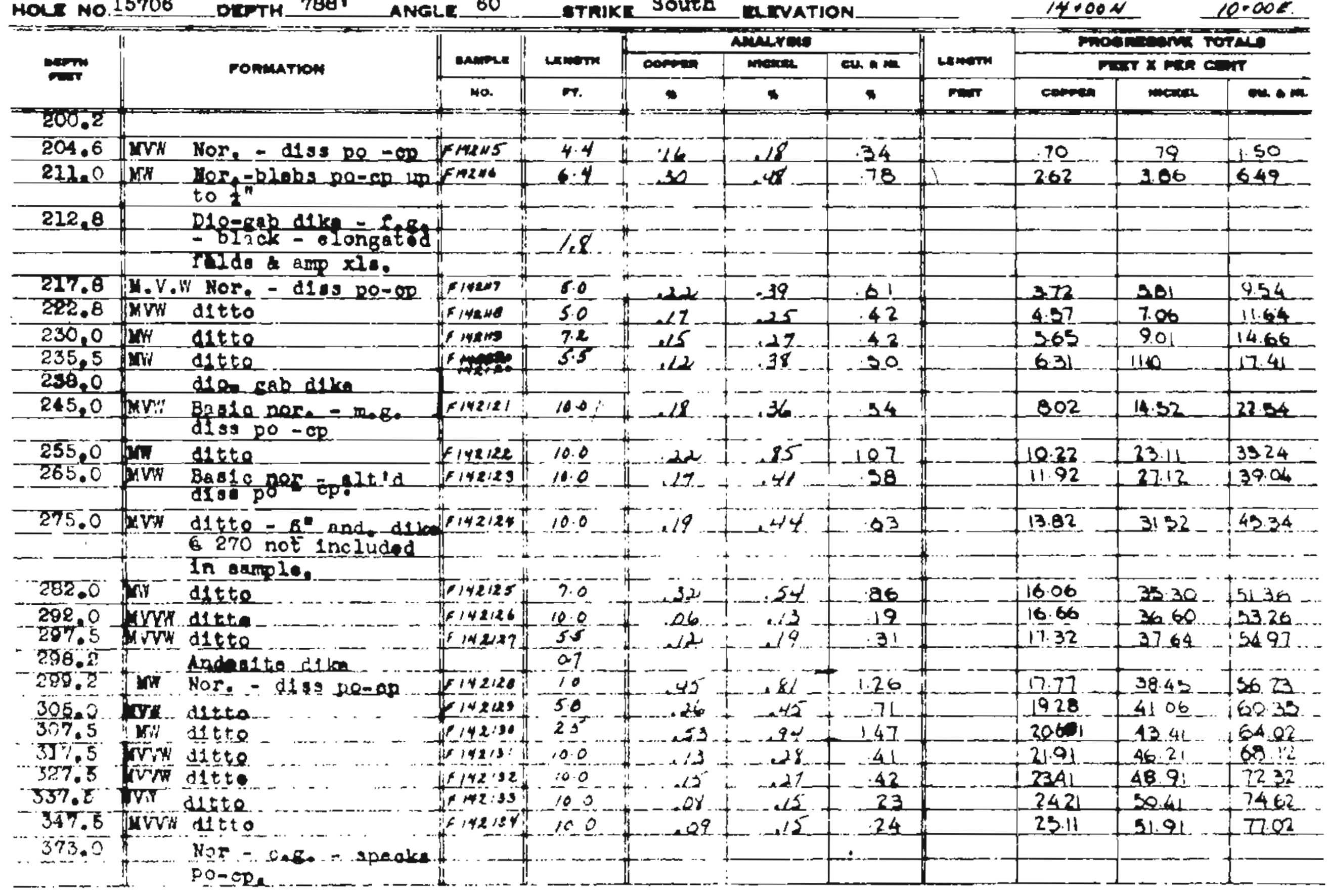




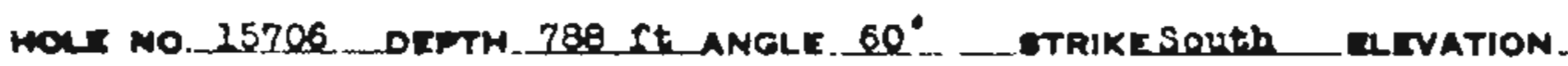

co-onernat:

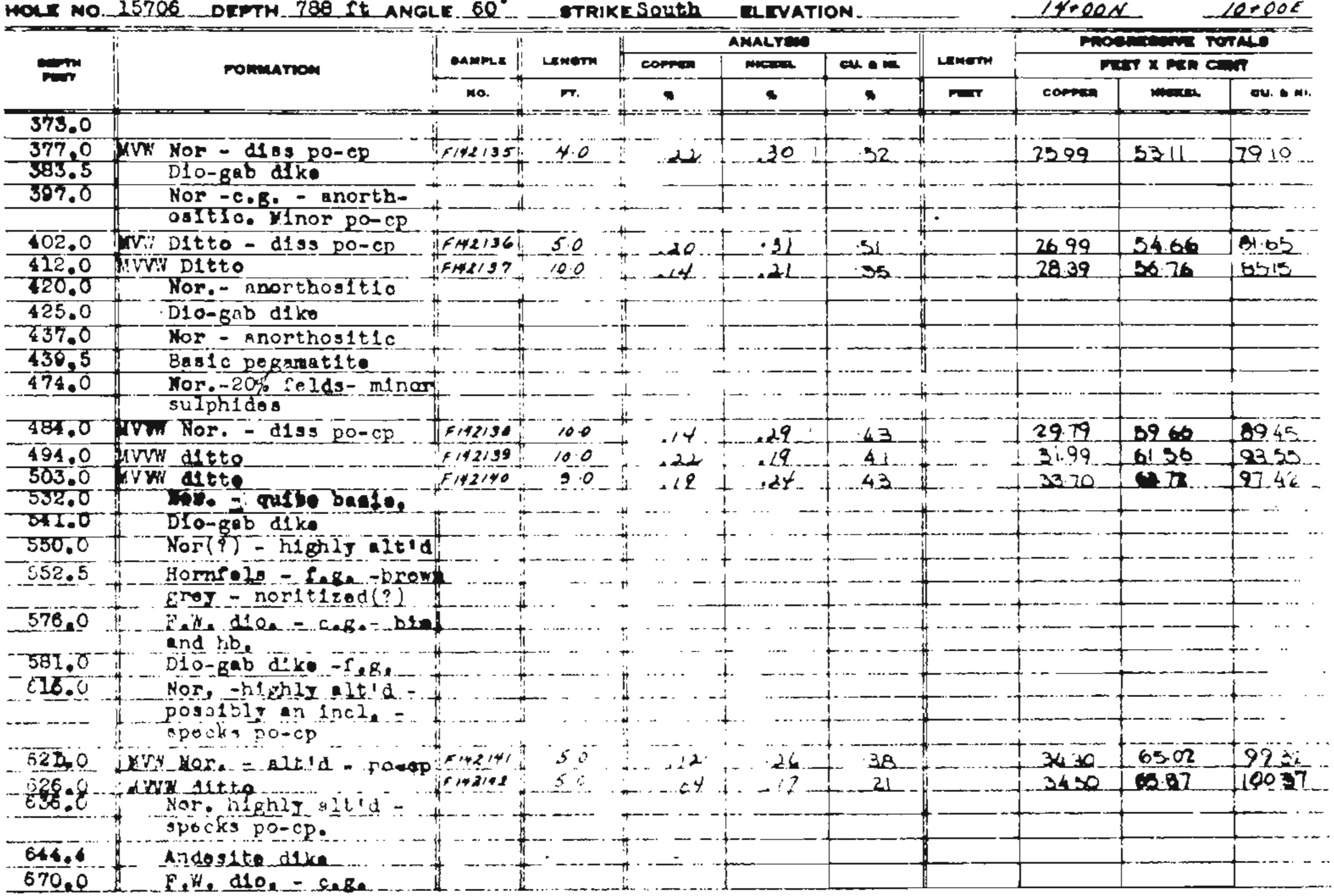

A 
Math No..

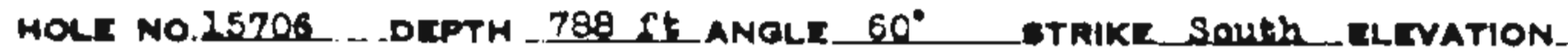

COCOMANATE:

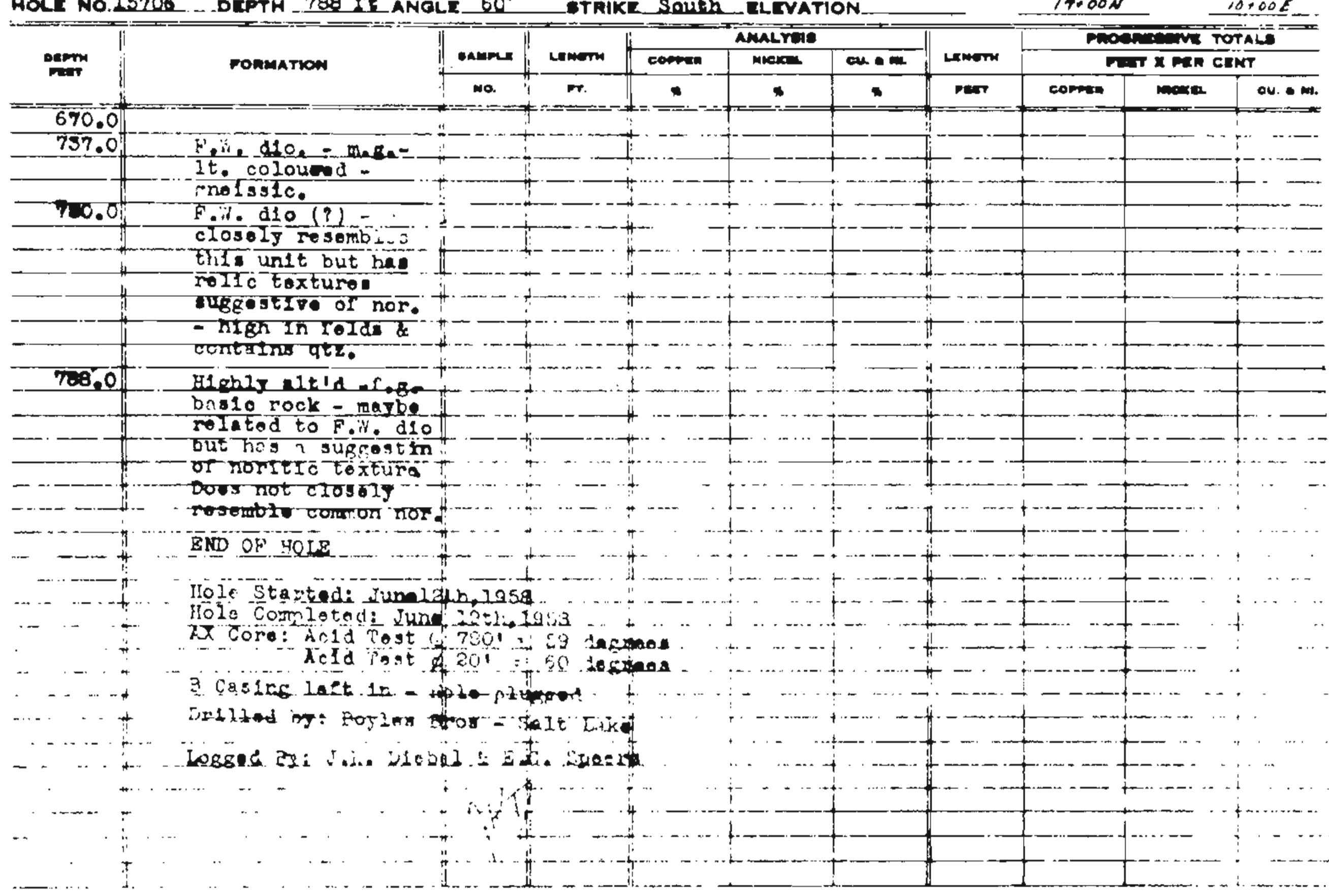




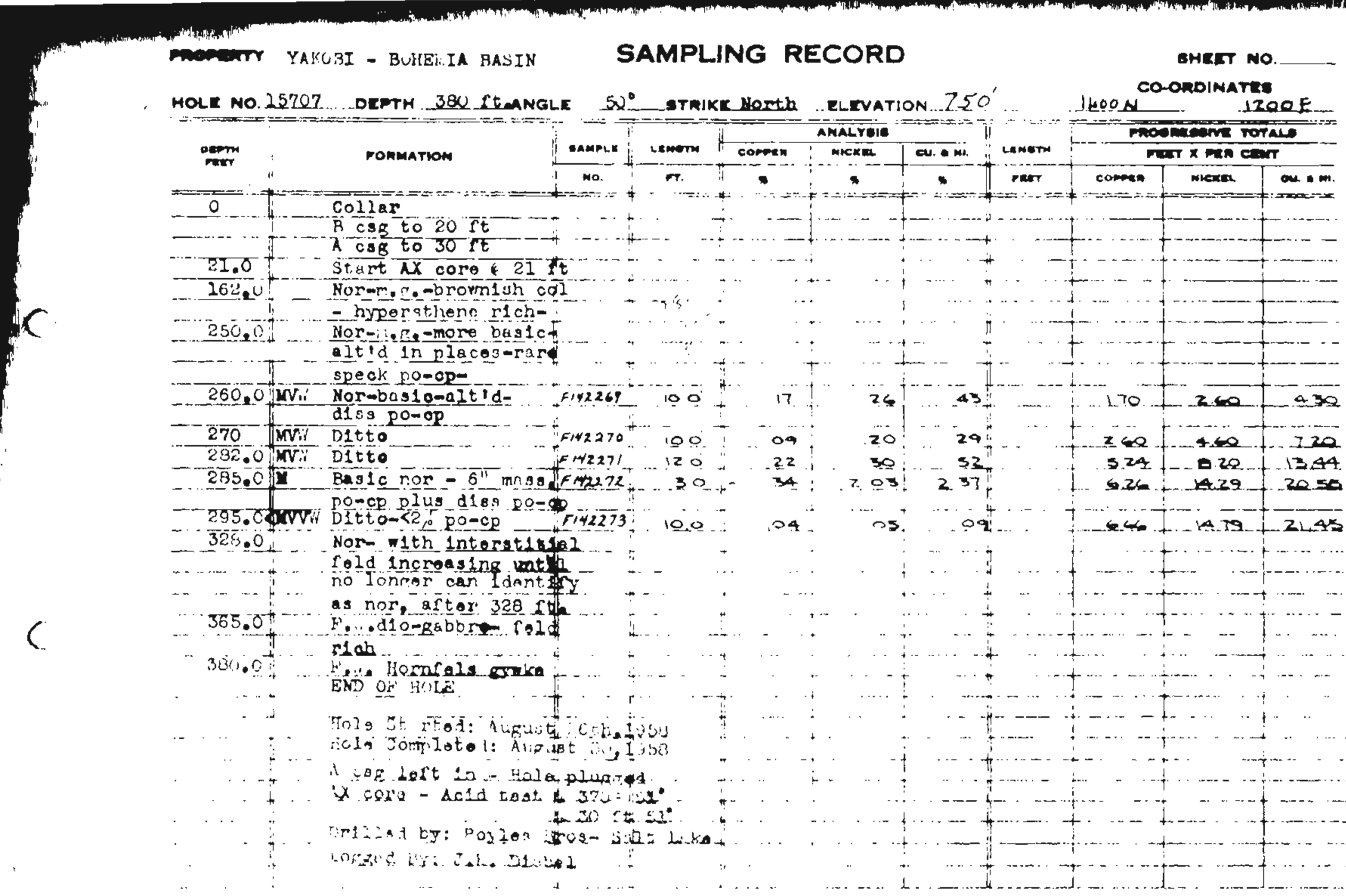


HOLE NO. 15700 DEPTH 94I rt ANGLE

arake wrth

ELEVATION Z7: ANAL Y=19

mekrir T cu. a m.

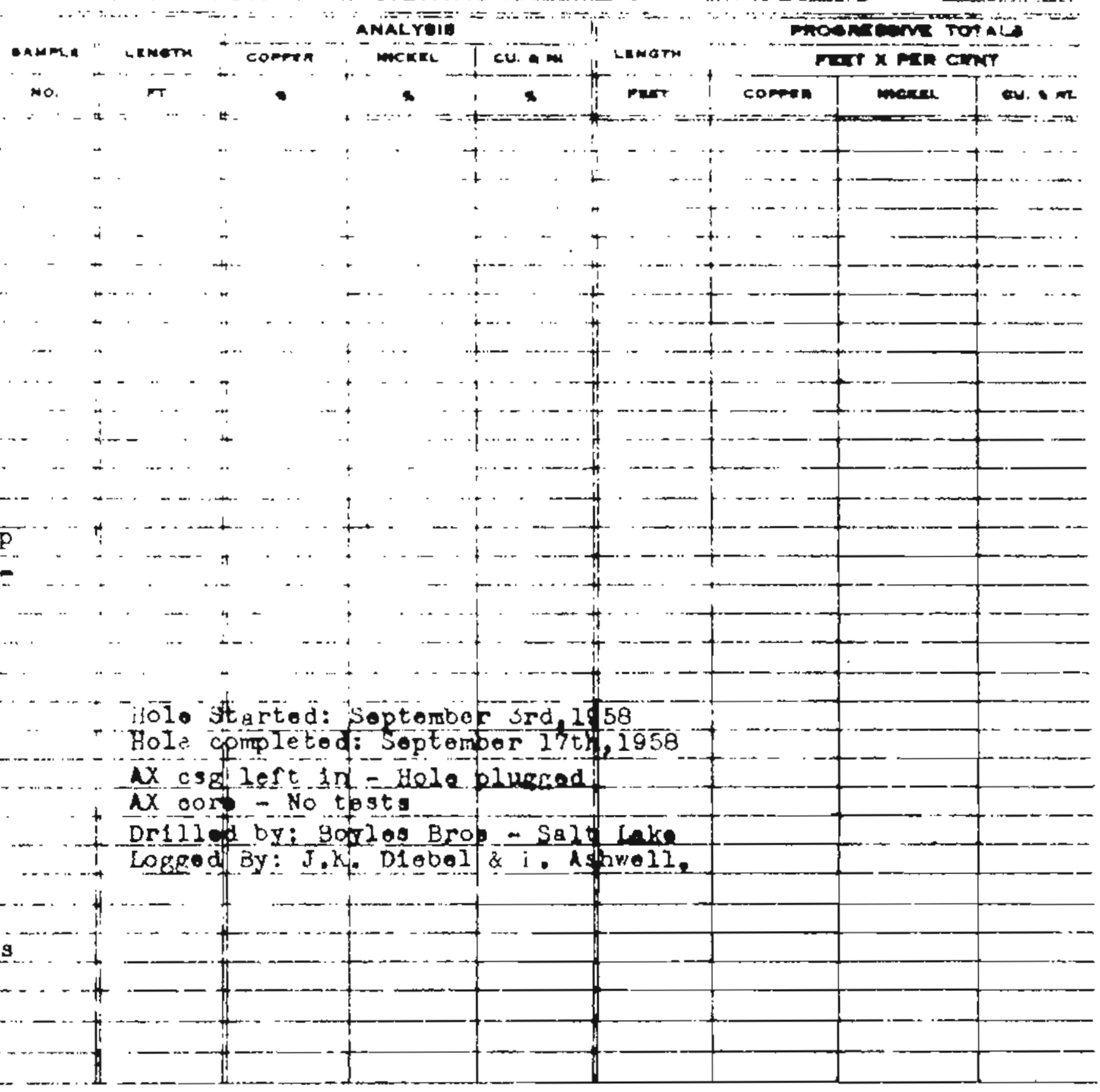
COOADINATE: OQN

S S 195 Hole cpmpletod: Sopterbor $27 \mathrm{th}, 1958$ - No tost Det

DHRT NO. I

$$
\operatorname{iom}
$$

monmation

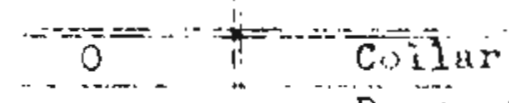

- . . . . B B tu

2द. A ist at coro

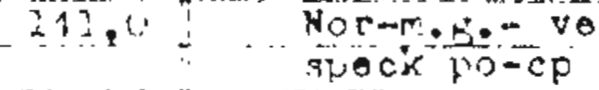

I4f Pas e nor-altid

-

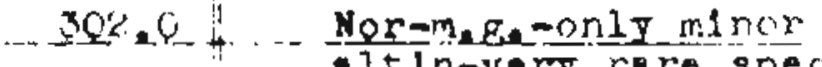

. .... .... ... altin-verg raro spect

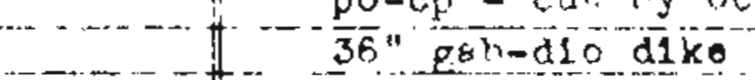

208.0 Nor-more o $1 \mathrm{c}$

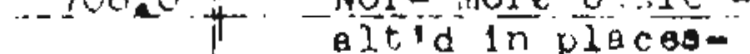

- ...... - vory rare snock po-to

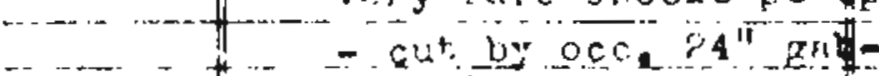

7 rat sof dikes. alteratiot

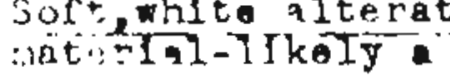

mo. sesperting

3as $1 \mathrm{c}$ nor $=$ hlgh!y ile' $\ln$ glaco: $=$ roro specks po-po-

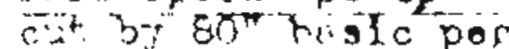

$$
\text { - } 770 \text { TE. }
$$

4lo Mi. tee one of the rab-li dikes but lt mors clusely reserbiss $\therefore$ d1o.

$\therefore \because$ U H L

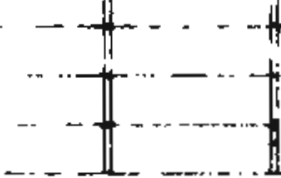

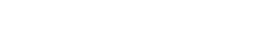




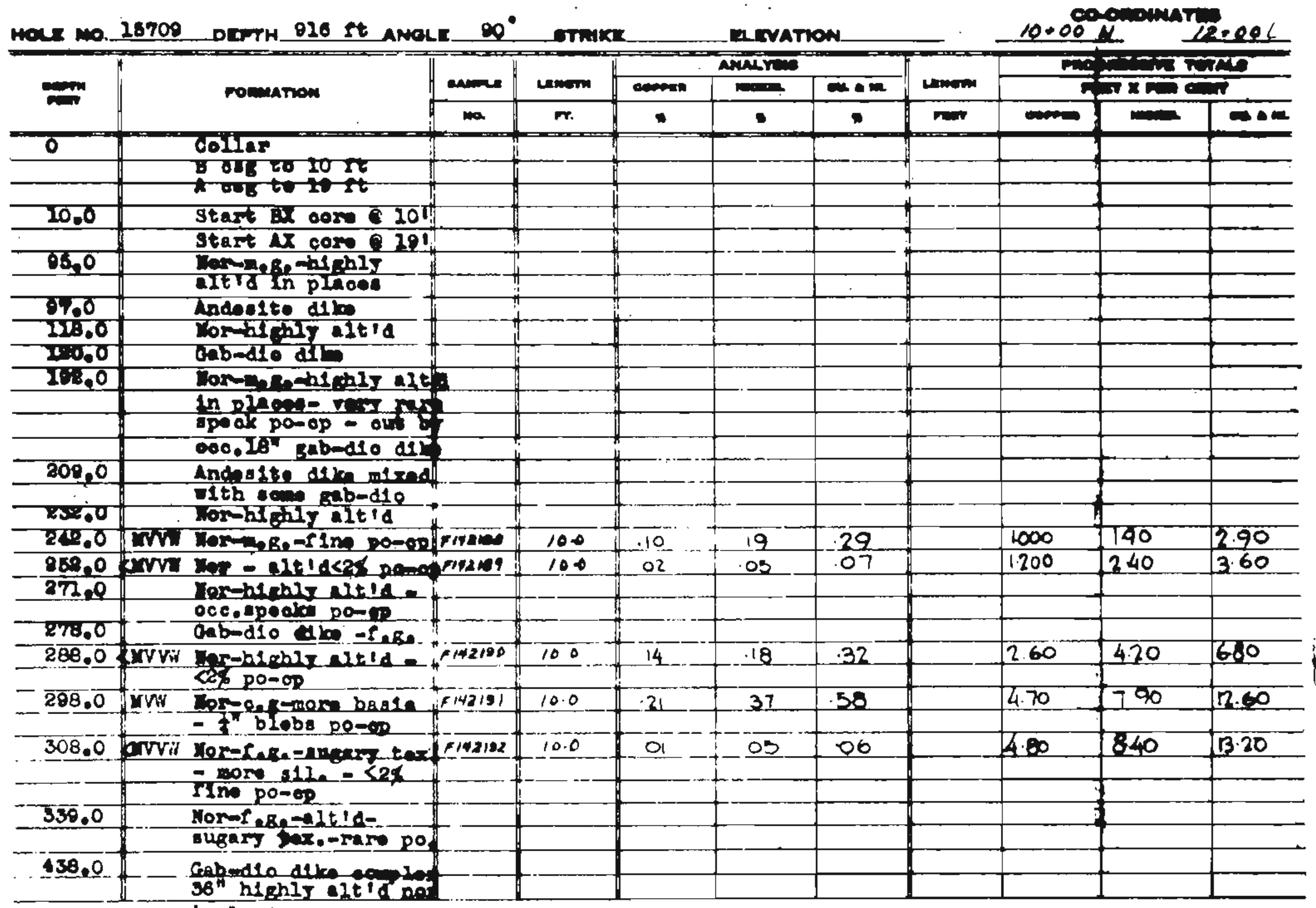




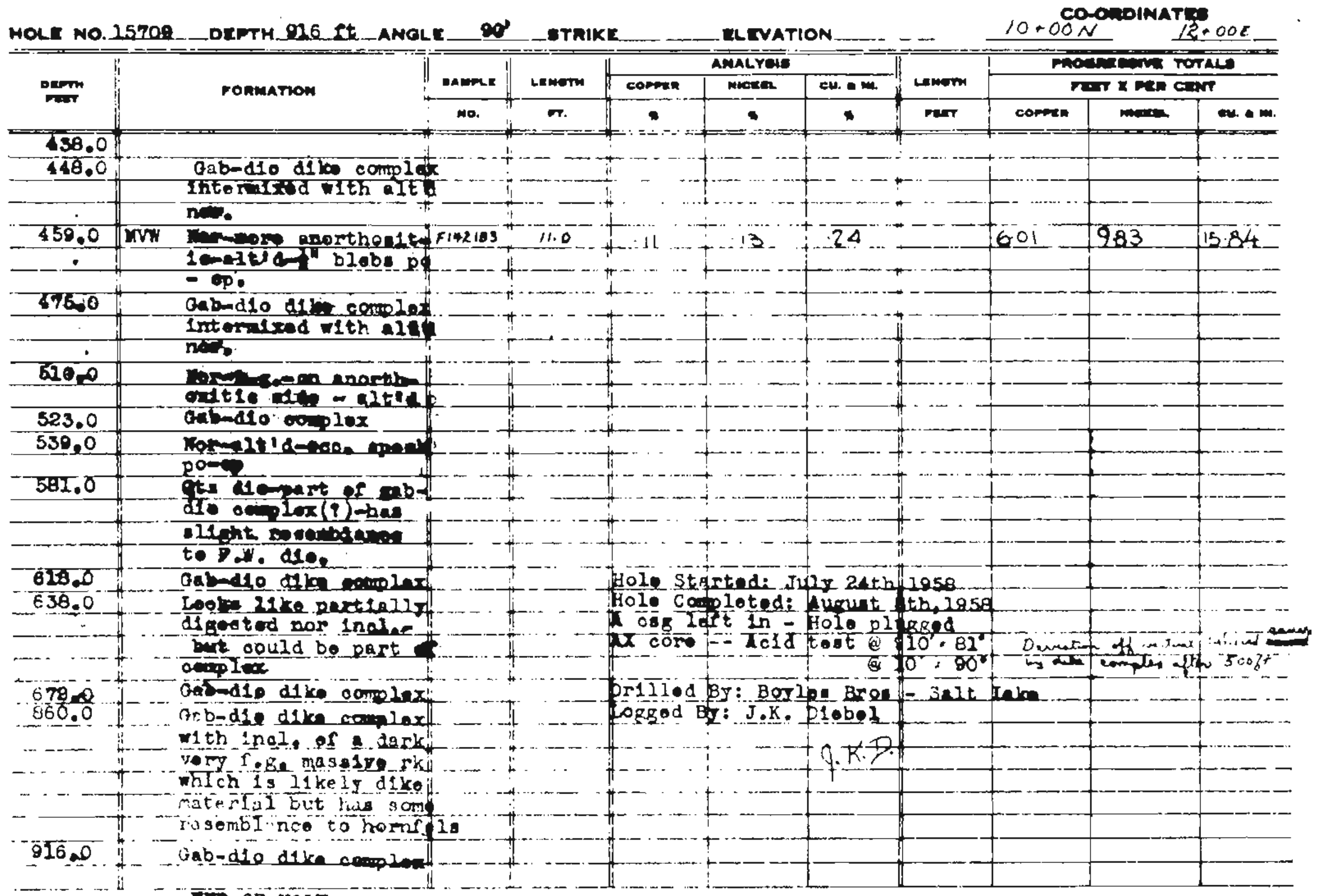

Dis of hoL: 


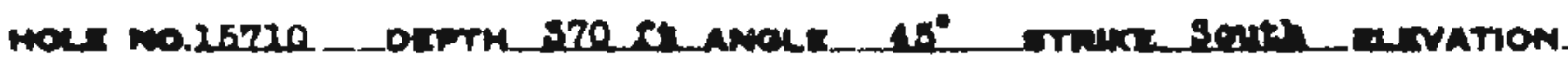
co-ominatra

\begin{tabular}{|c|c|c|c|c|c|c|c|c|c|c|}
\hline \multirow{2}{*}{ imi } & \multirow[b]{2}{*}{ momintion } & \multirow{2}{*}{$\frac{\operatorname{man}}{\text { man }}$} & \multirow{3}{*}{$\frac{\operatorname{Ln}}{\mathrm{m}}$} & \multicolumn{3}{|c|}{ Nuine } & \multirow{2}{*}{ m } & \multirow{2}{*}{\multicolumn{3}{|c|}{ manom }} \\
\hline & & & & anding & $-m$ & $=-1$ & & constin & & \\
\hline 0 & Coliar & & & & 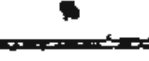 & & & & & \\
\hline \multirow[t]{2}{*}{10,0} & $B$ ect $-\operatorname{stant} B x$ & & & & & & & & & \\
\hline & $00 \mathrm{re} 610 \mathrm{Kt}$ & & & & & & & & & \\
\hline \multirow[t]{2}{*}{20.0} & 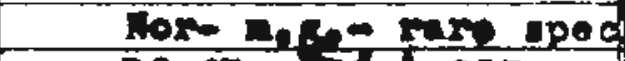 & & & & & & & & & \\
\hline & po-ope and 2 ong & & & & & & - & & & \\
\hline \multirow[t]{2}{*}{30.0} & Ior-buaching wort & & & & & & & & & \\
\hline & backo & & & & & & & & & \\
\hline \multirow[t]{2}{*}{73.0} & 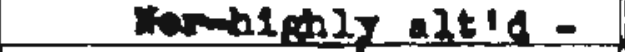 & & & & & & & & & \\
\hline & rare spoake po-op & & & & & & & & & \\
\hline$\frac{79.0}{115.8}$ & Anderite ill & & & & & & & & & \\
\hline \multirow{2}{*}{215.8} & Dohldolentid. & & & $\dot{0}$ & & & & & & \\
\hline & rave opoake po-os & & & & & & - & & & \\
\hline \multirow{2}{*}{235,0} & abblels alt:de & & & & & & & & & \\
\hline & besio poge frold \& & & - & & & & 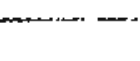 & & & \\
\hline & proxenel & & & - & & 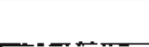 & & & & \\
\hline \multirow{2}{*}{246.9} & pan up & & & & & & - & & & \\
\hline & $p^{0}=00-16^{\circ}=$ & & & & & & - & & & \\
\hline \multirow{2}{*}{16.0} & 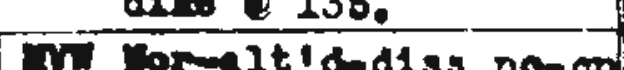 & & & & & & & & & \\
\hline & 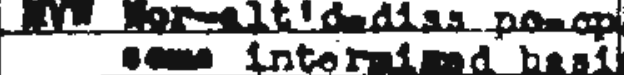 & restar & 100 & - 0 4 & .06 & .10 & & 40 & 60 & 1.00 \\
\hline \multirow{2}{*}{ - } & $\mathrm{pop}=10 \ln _{2} \circ I t$ & & & & & - & & & & \\
\hline & tho or semplo & & & & & & & & & 5 \\
\hline \multirow{2}{*}{206,0} & Gab-d10 dike couplax & & & & & & & & & $-\ldots$ \\
\hline & interned at wh som & & 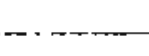 & & - & - & & & & \\
\hline \multirow{3}{*}{$\begin{array}{l}214.0 \\
224.0 \\
254.0\end{array}$} & Hor-mge - rers nom & & & & & & & & & \\
\hline & 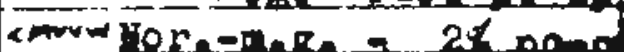 & $(\pi+2146$ & 10.0 & 15 & 25 & 40 & & 1.90 & 3.10 & 5.00 \\
\hline & 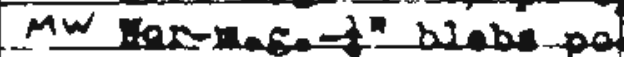 & 214297 & 100 & 36 & 42 & 78 & & 530 & 7.30 & 1280 \\
\hline \multirow{3}{*}{$-\overline{244} e^{0}$} & ope. & & & & & & & & & \\
\hline & in fex & FlWENI & 10.0 & 27 & 33 & .60 & & 8.20 & 1060 & 1880 \\
\hline & babia-1/8 blobs & & & & & & & & & \\
\hline
\end{tabular}




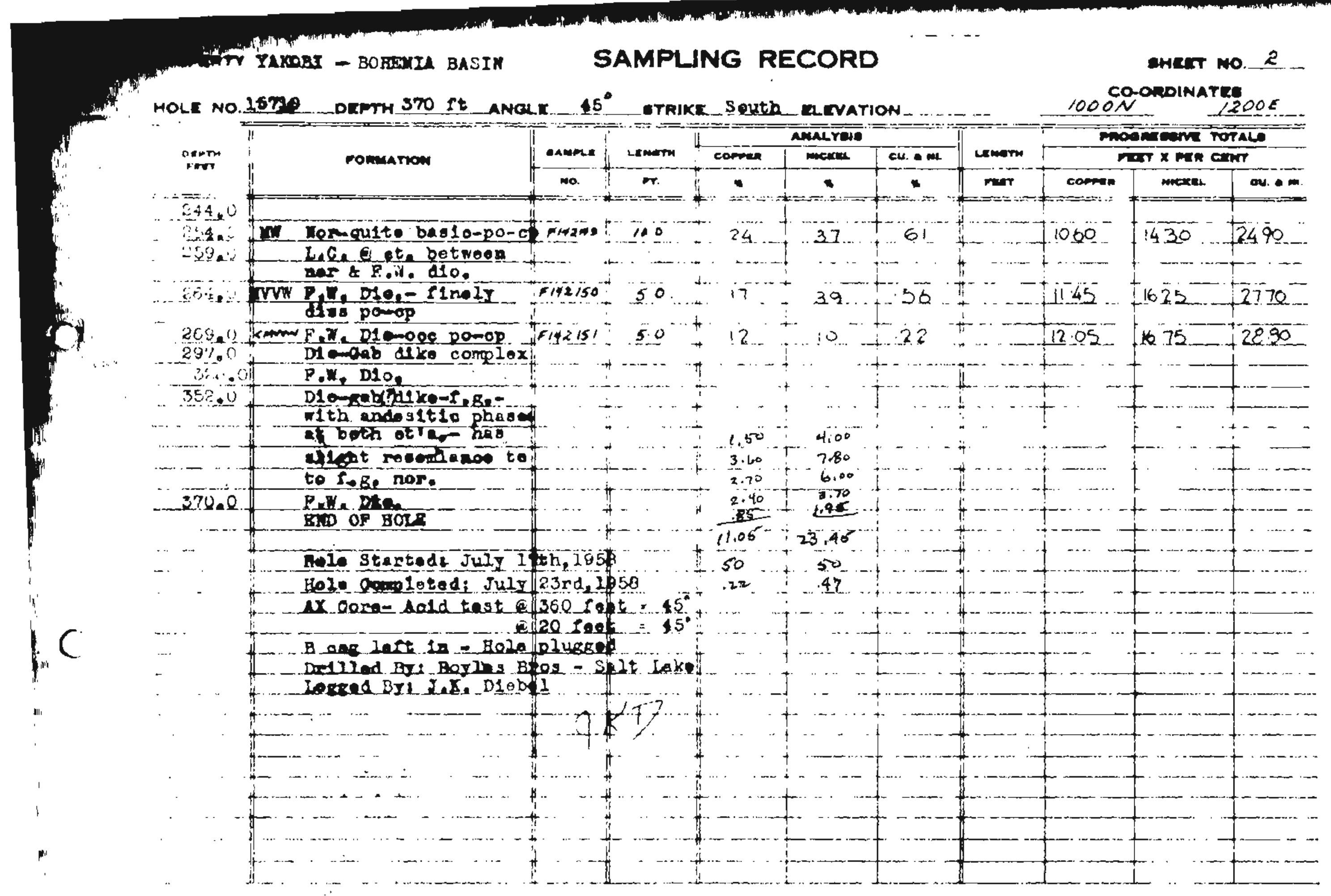


entit No._ /.

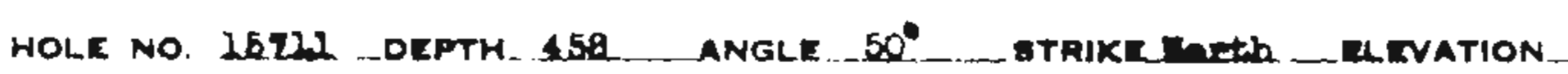

Co-ondinate

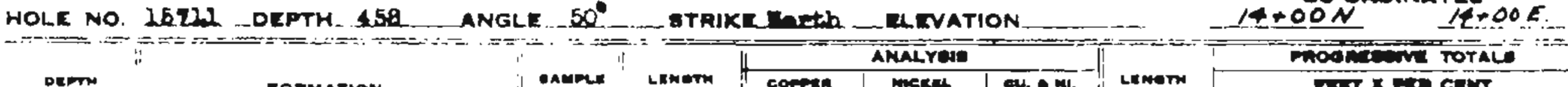

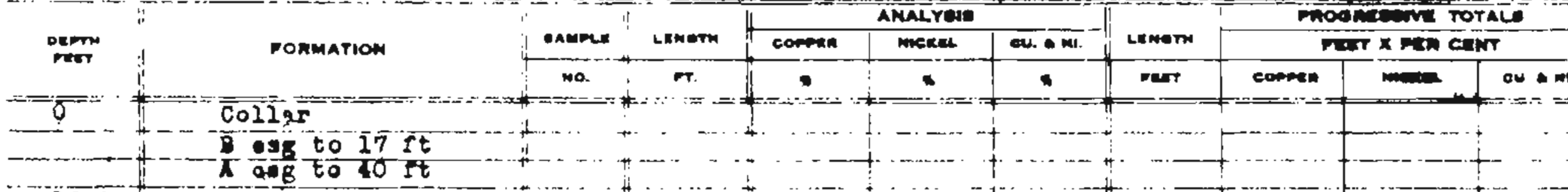

13.0
59.0

$68.07-\mathrm{Bas13}$ pego

159.0 Noraltid to highI

- altd = rere spechs

…

171,0 0 Bas10-pog.

201.0 Mor-h1 ghly altid-

226.0 H-Dio d ke = mage =

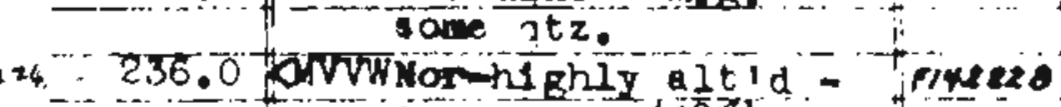

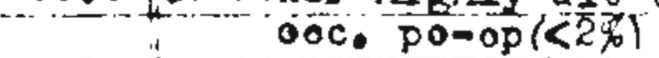

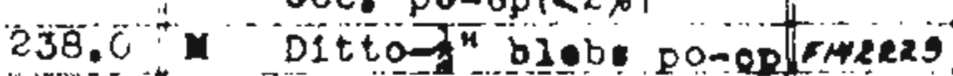

242.0 Arrw Nor-alt ld= dias parep meupo

242.251 .0

261.0

Besc pere

- Bese deta.

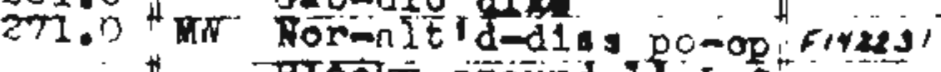

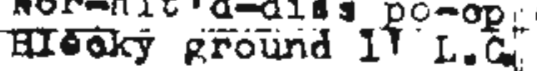

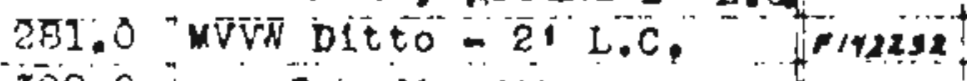

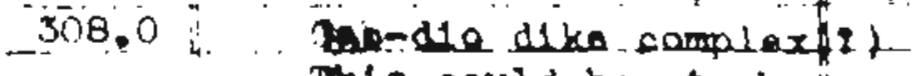

Th1 oguld ba ot phope

c. folloning dio,

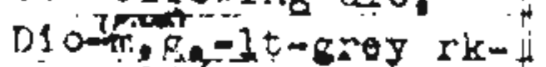
over atz (?) -cut by occ. $24^{* 1}$ ret dio.dike as at 3301 ( END OF $: \mathrm{OL}$ 


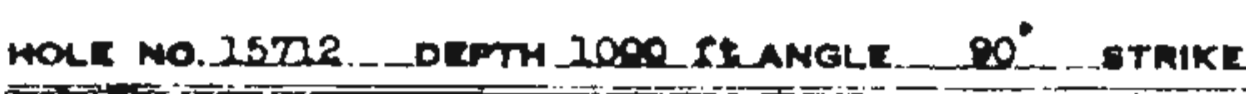

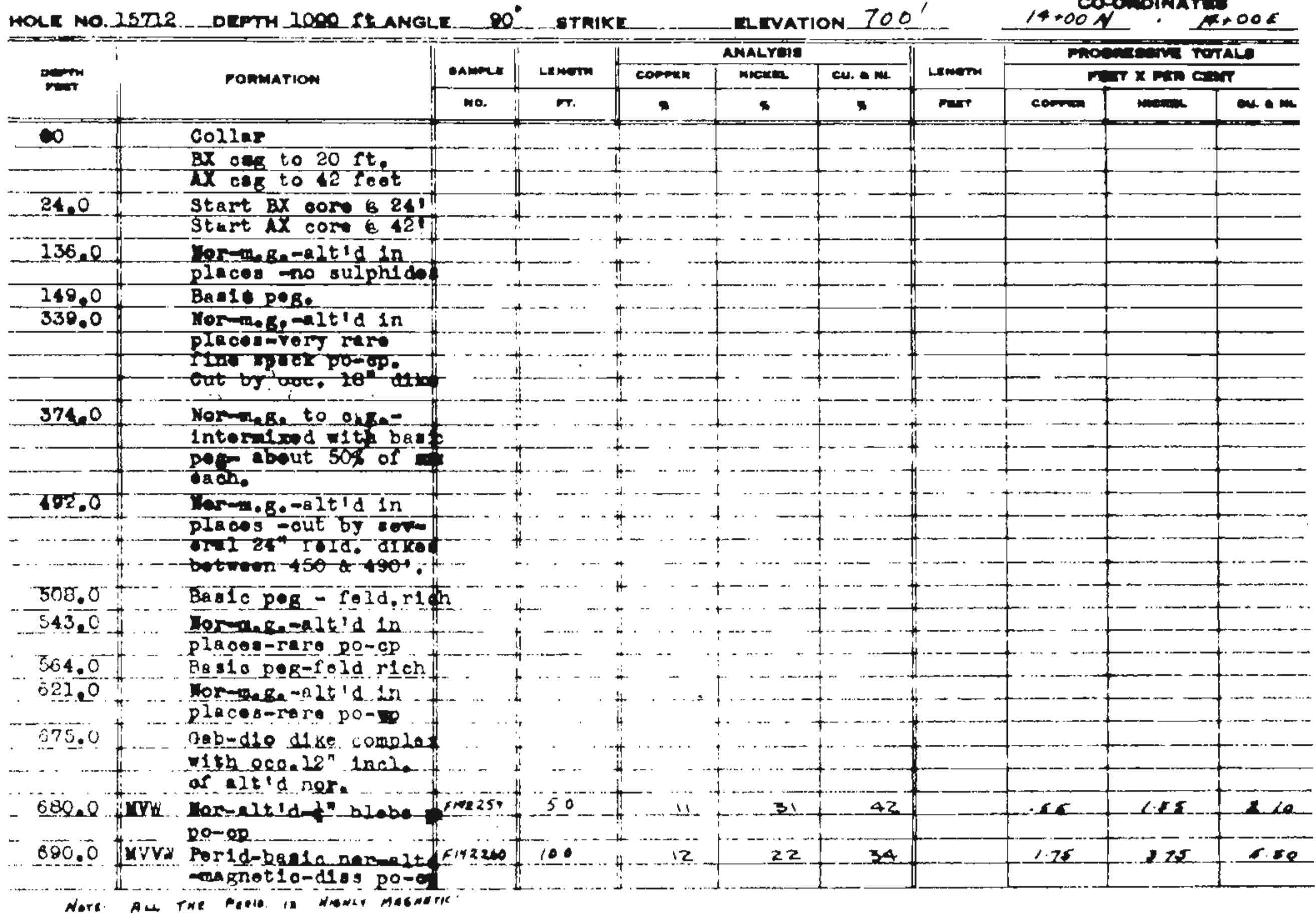




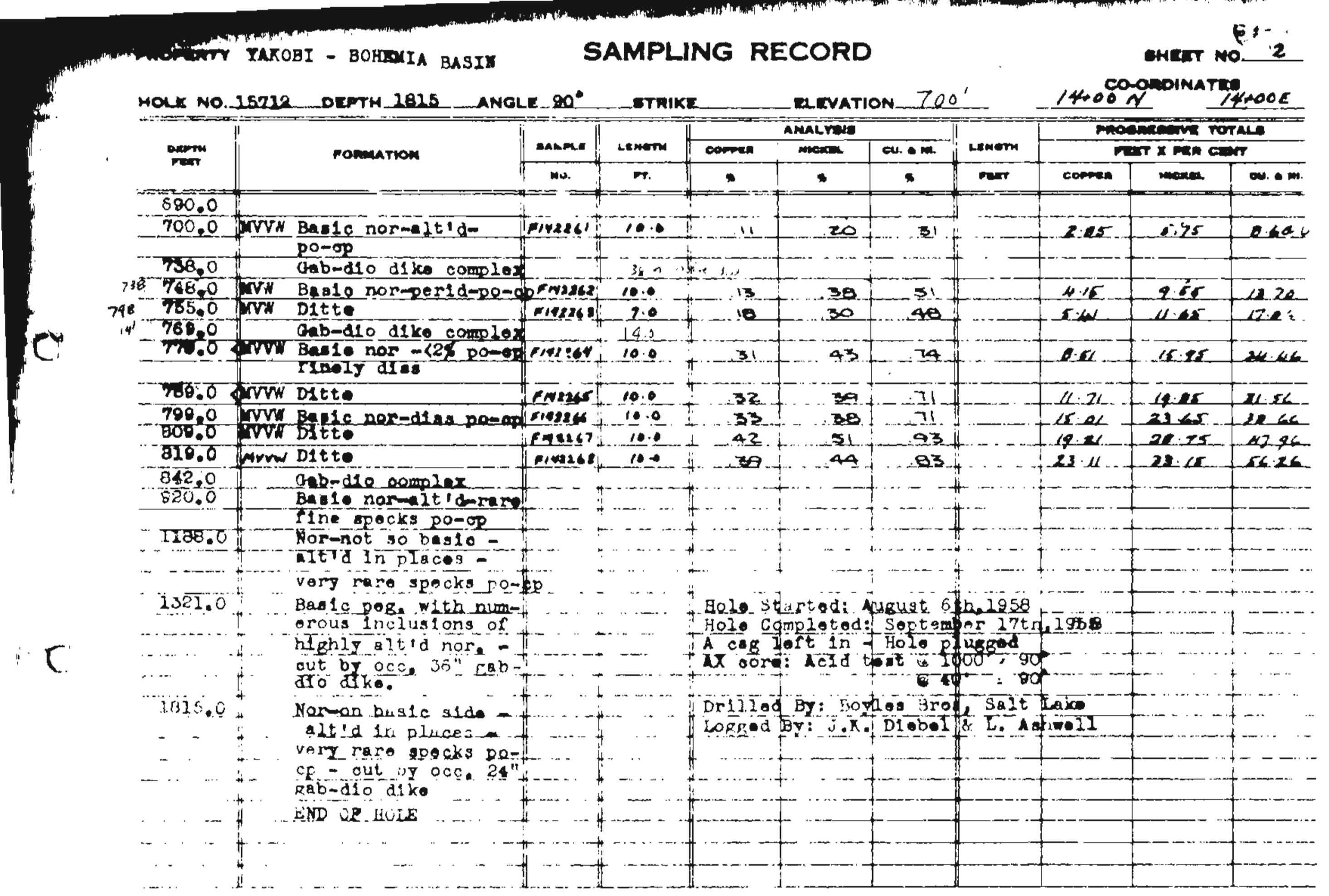




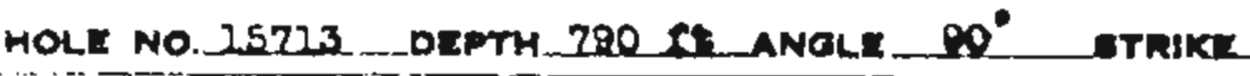

ARATION

comin

Anatrote

$0=\frac{\operatorname{coliax}}{\mathrm{B} \text { cesg to } 20 \mathrm{rt}}=$

11,0 Start $1 X$ cora WI TaA

135.0 xor-mo-minor altin

- In In acose cut by occ.

601.0 Noranorobano-altid

601.0 Noranoro basio-aitid

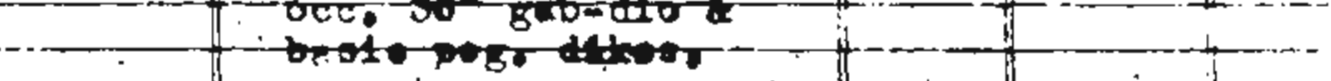

6I8.0 Nor Intêryxed with

- I8. - Nor interuxed with

703.07 - Hor en basto sta

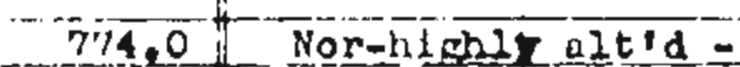

- Intermixed $1 \mathrm{th}$ reldr $\mathrm{dch}$

तIk rock

.. TBIfo Norhighly alt

790,0 oab-dio dike complox

... H OD HOL

… Hol startodiuguet 3 to 1858

- .. Folo comptot: 3optember 2ndh 195

A cog Loft In- Bolo PItsged

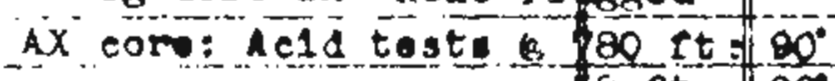

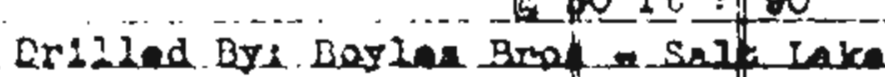
Logerd BY: J K. Dlebel

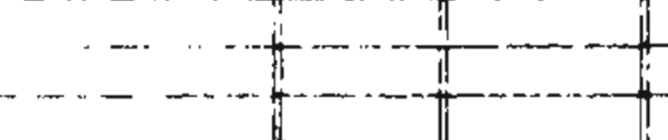

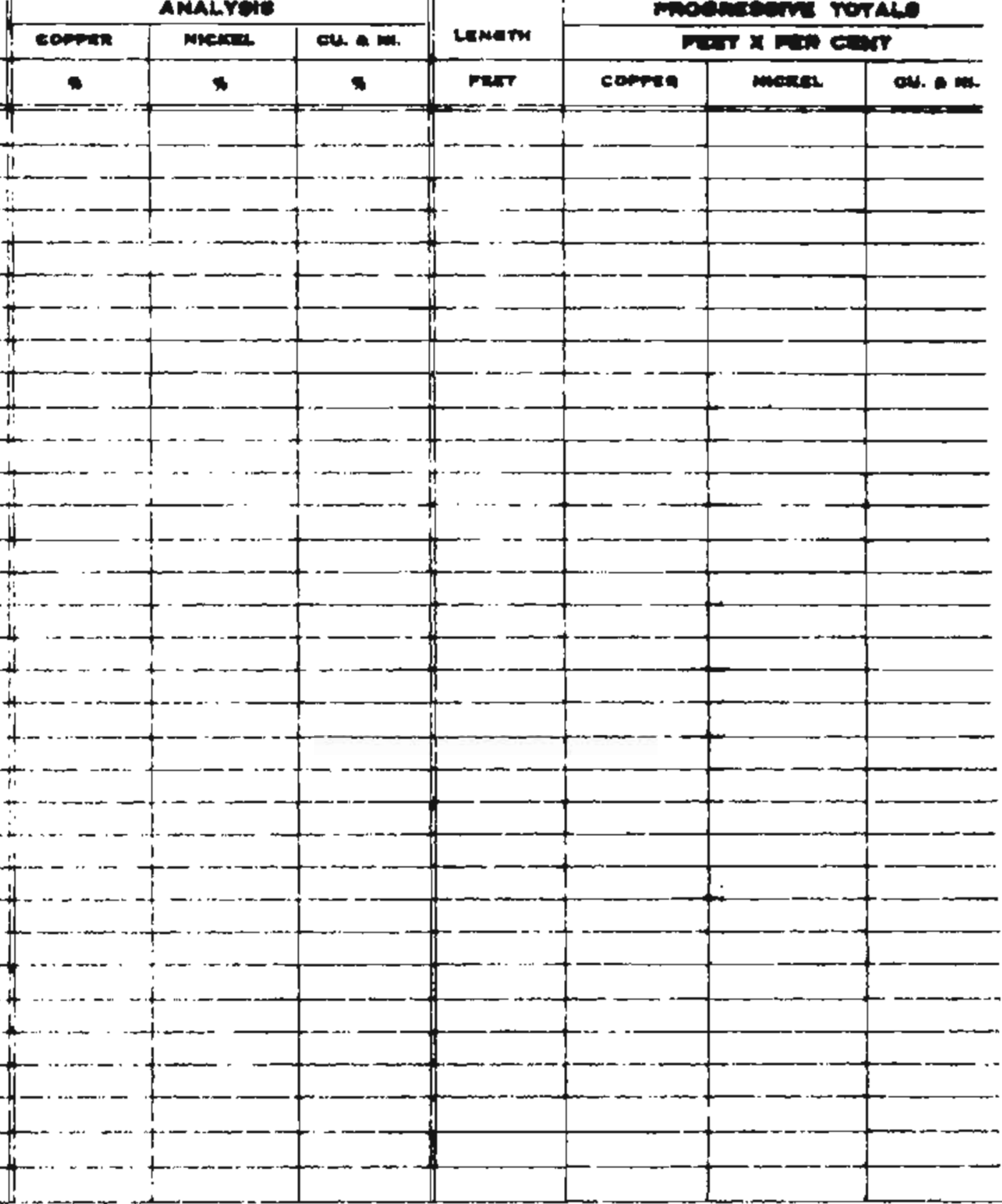

morotom Torato

Mis x ats cont 


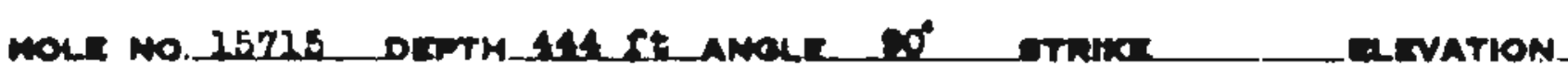
Nerive

$0=$ Gol2ar

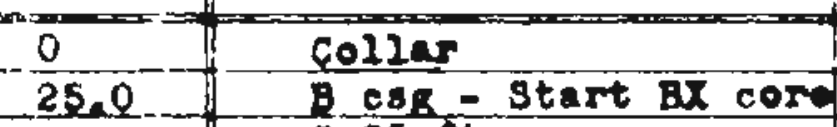

30.0 Nor-highly alt'd to

- actinolito - ind

Cag - Start dX $00 \%$

180.0 Nor-mes. quite bas1

- - - highly itid in

- $6 \mathrm{~B}$ B

- oec speck po- ope

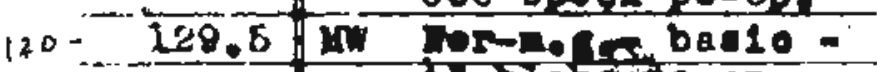

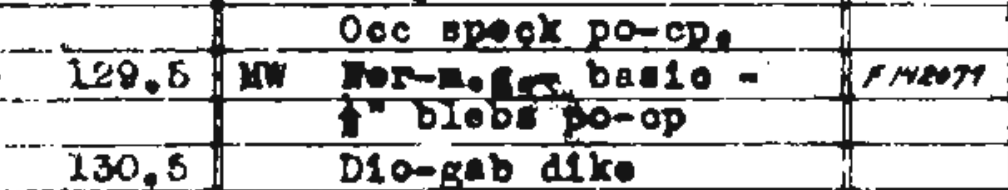

240.0 nvw Hor-m.8. to c.8.

1600 balo- in blobs po-c

150.0 urm pitto

- 16000 KVW D1tto

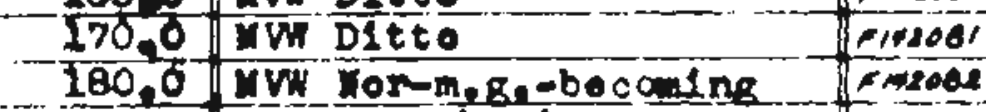

- VoF basic - po-op

Nivi Ditto

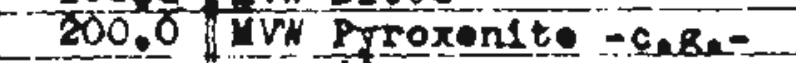

270.0 .

210. 215.0 LVH Ba10 yor beconde

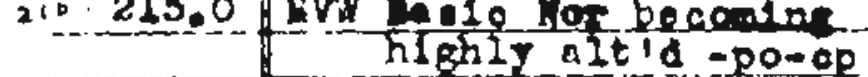

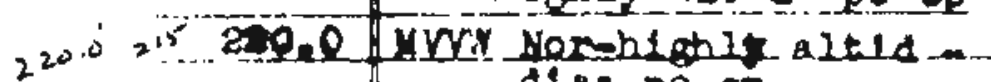

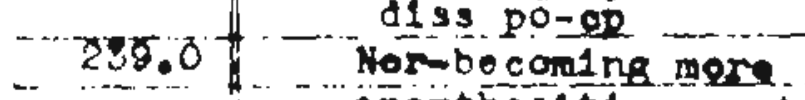

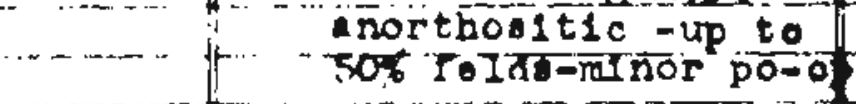

\begin{tabular}{|c|c|c|c|c|c|c|c|c|}
\hline \multirow{3}{*}{ annen } & \multirow{3}{*}{$\frac{\ln m m}{m .}$} & \multicolumn{3}{|c|}{ Nurros } & \multirow[b]{2}{*}{ Mnorment } & \multirow{2}{*}{\multicolumn{3}{|c|}{ 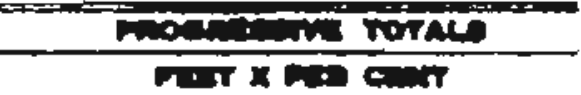 }} \\
\hline & & \multirow{2}{*}{ 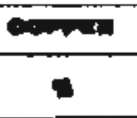 } & \multirow{2}{*}{$-\frac{1}{1}$} & \multirow{2}{*}{$\frac{\pi}{6}$} & & & & \\
\hline & & & & & $\boldsymbol{m r}$ & capren & 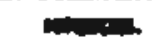 & 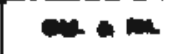 \\
\hline & & & & & & & & \\
\hline & & & & & & & & \\
\hline & & & & & & & & \\
\hline & & & & & & & & \\
\hline & & & & & & & & \\
\hline & & & & & & & & \\
\hline & & & & & & & & \\
\hline & & & & & & & & \\
\hline & & & & & & & & \\
\hline & & & & & & & & \\
\hline & & & & & & & & \\
\hline & & & & & & & & \\
\hline 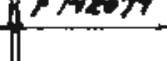 & 9.5 & .2 & -19 & .02 & & 209 & 3.00 & $\operatorname{sen}$ \\
\hline & 10 & & & & & & & - \\
\hline $4 \overline{142 m \delta}$ & .5 & .19 & -31 & 51 & & 380 & 6.94 & 1074 \\
\hline & & & & & & & & \\
\hline Fiseoso & 100 & il & 19 & 30 & & 4 우응 & 6.84 & $13 \widehat{16}$ \\
\hline$F+10000$ & 10.0 & $B$ & 2 & 34 & & 620 & 1094 & 1714 \\
\hline $0101006 t$ & 100 & 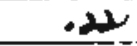 & +14 & 36 & & 840 & 1434 & 2274 \\
\hline Eneode & 10.0 & $=y_{0}$ & -.49 & 89 & & 1240 & 1924 & 3104 \\
\hline Ex+1003 & $2 \cos 40$ & $=7$ & & -7 & & -1 & $0=-2$ & $t=0$ \\
\hline $6 / 4200$ & $\frac{100}{10.0}$ & 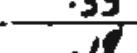 & -140 & 13 & & 1270 & $62 \leq 4$ & 3894. \\
\hline & & & & & & 1.40 & 120 & {$[45.34$} \\
\hline F/72000 & 100 & -4 & At & 33 & & 19.10 & 29.34 & 68.64 \\
\hline$f+4000$ & $\Leftrightarrow \sigma_{r} 0$ & $\therefore$ & $.3 h$ & 64 & & 398 & $3+4$ & 5504 \\
\hline & & & & & & 1,18 & $x, \infty$ & \\
\hline-10201 & 105,0 & -21 & $=-40$ & 63 & & 24.20 & 32.4 & 61.34 \\
\hline & & & & & & 21,65 & 33.4 & \\
\hline & & & & & & & & \\
\hline & & & & & & & & \\
\hline & & & & & & & & \\
\hline
\end{tabular}

$1 \% 20$

220

$\frac{36}{180} \cdot 22$

$33(20)(81)$

6.54 


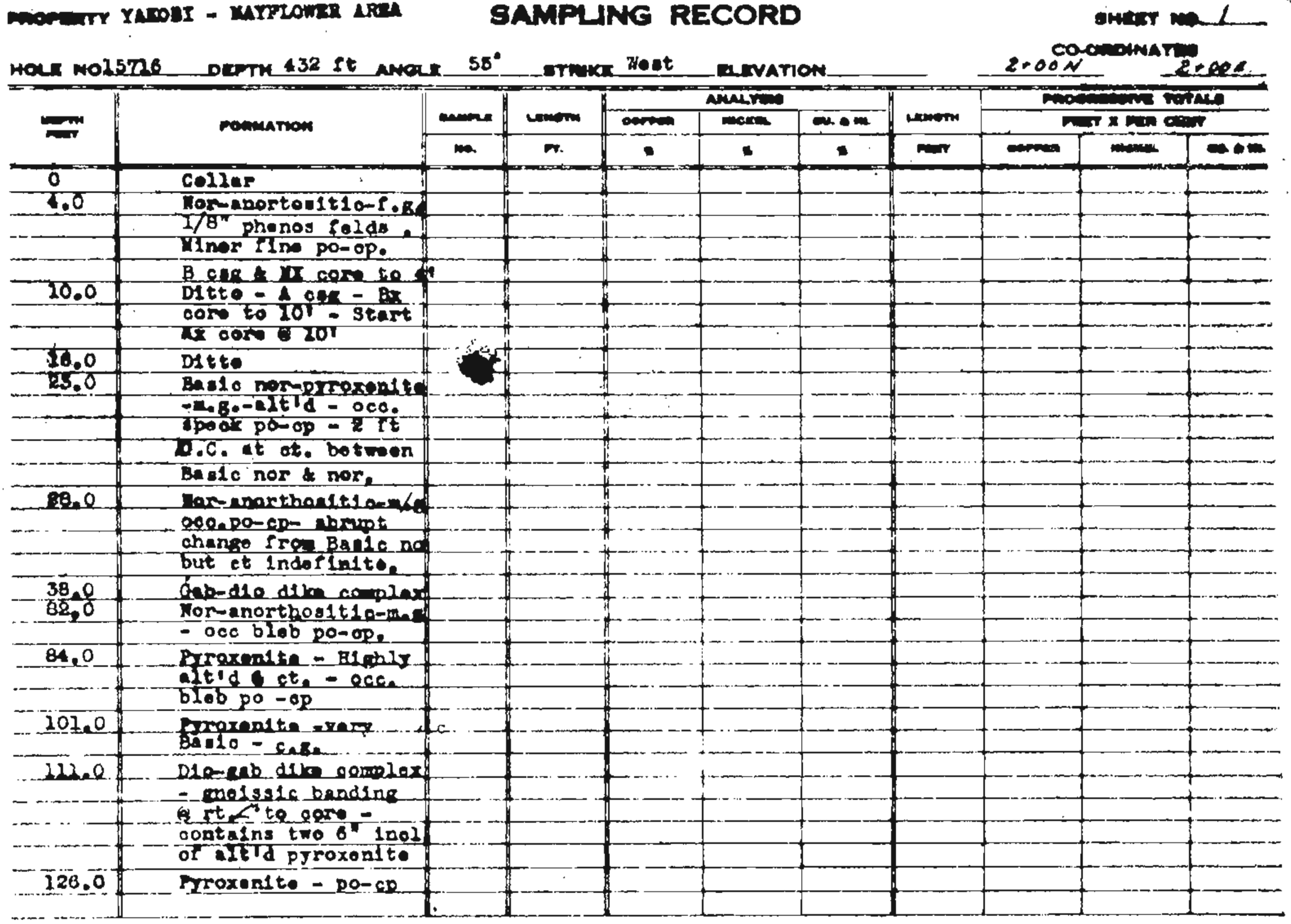


otrike ilost

ELIVATION CO-ORDINATEA

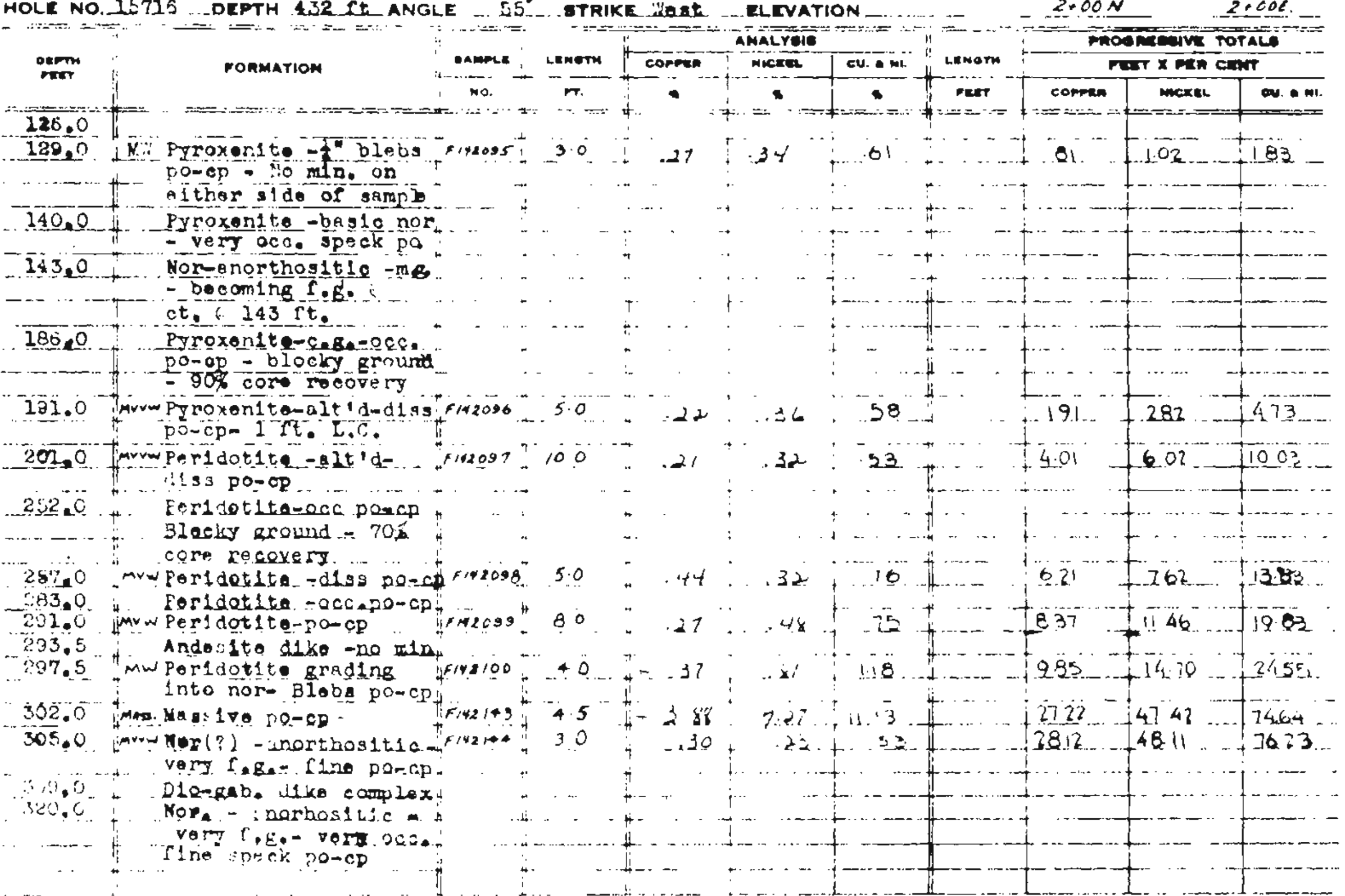




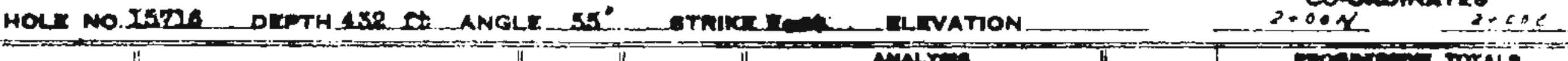
co-owomatre

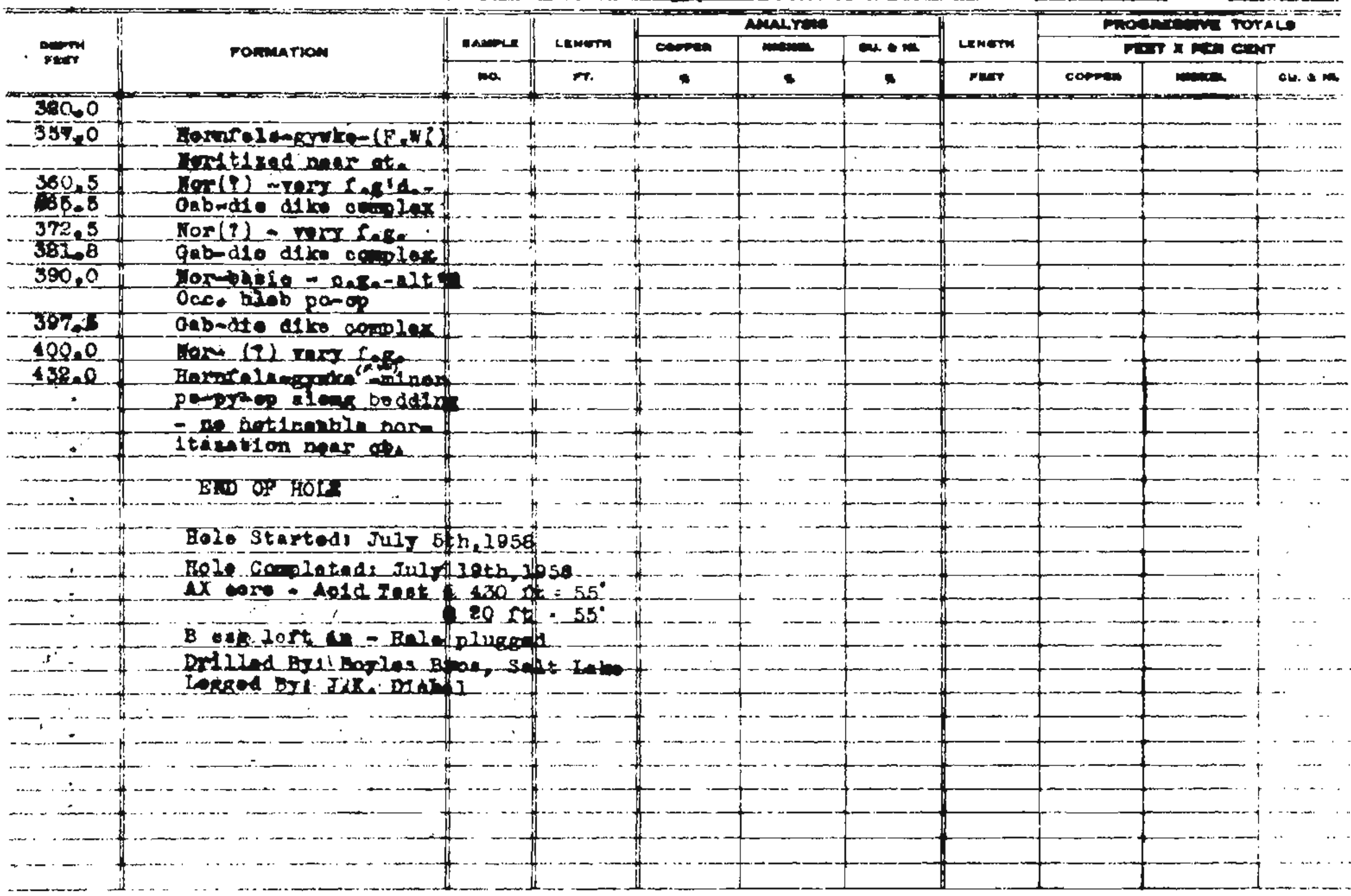




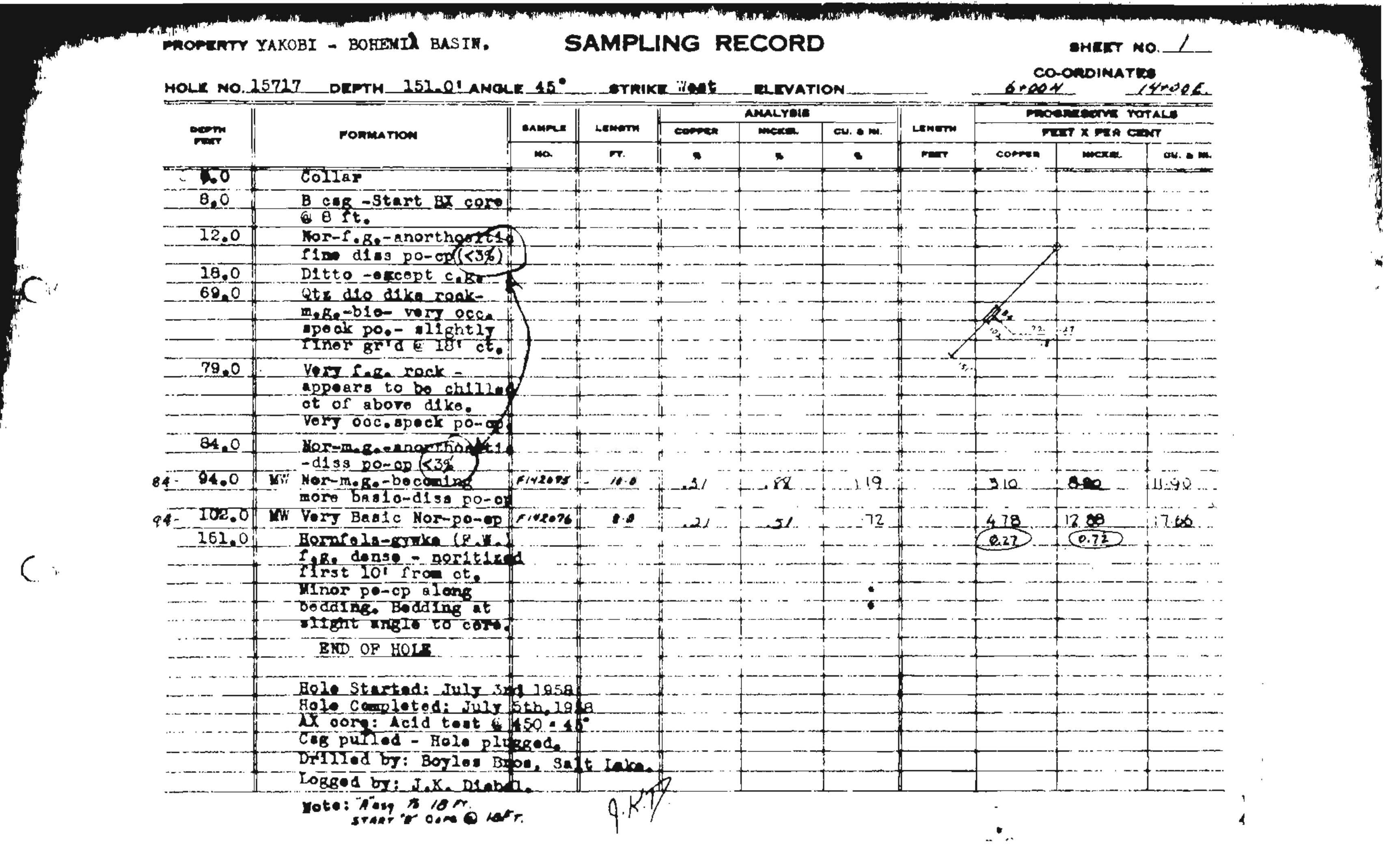




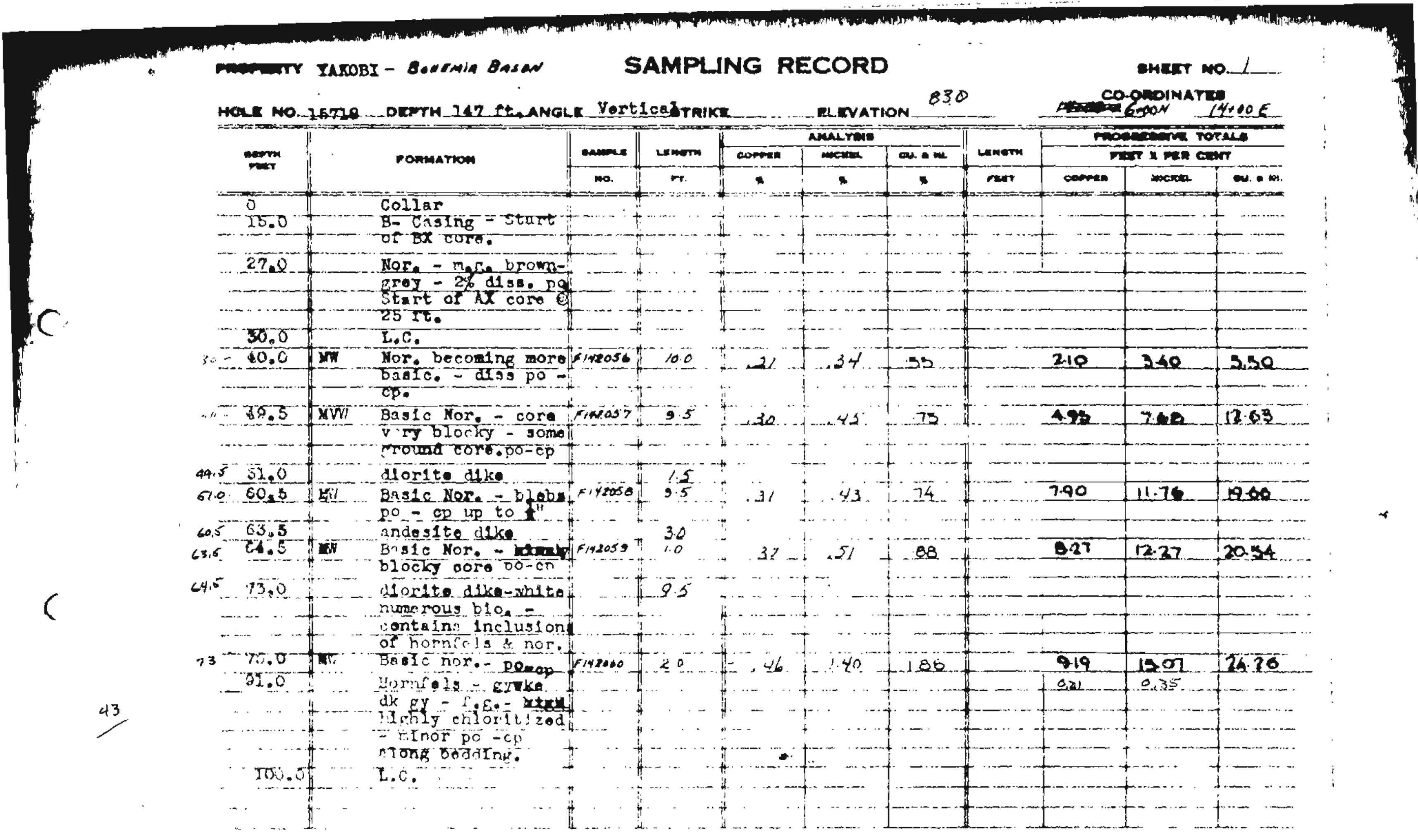


matr no. 2 .

HEN N WO 15719 _.DEPTH_147.0 ANOLE Vart. OTRIKE

ELINATION coconoinatze

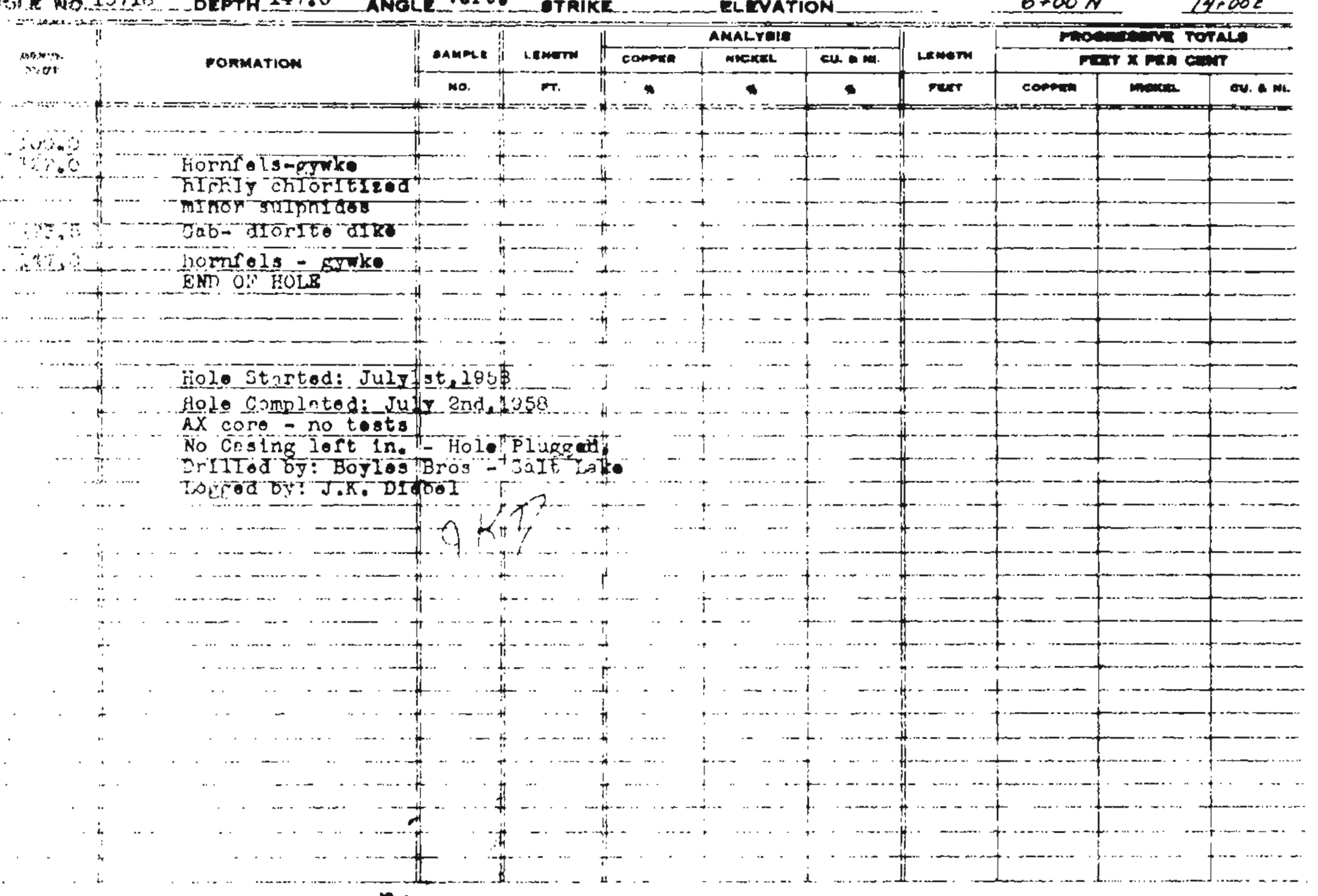

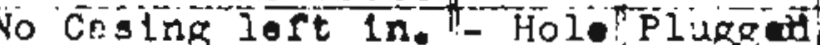

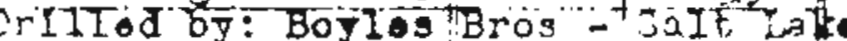

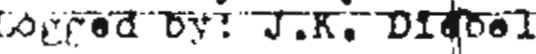




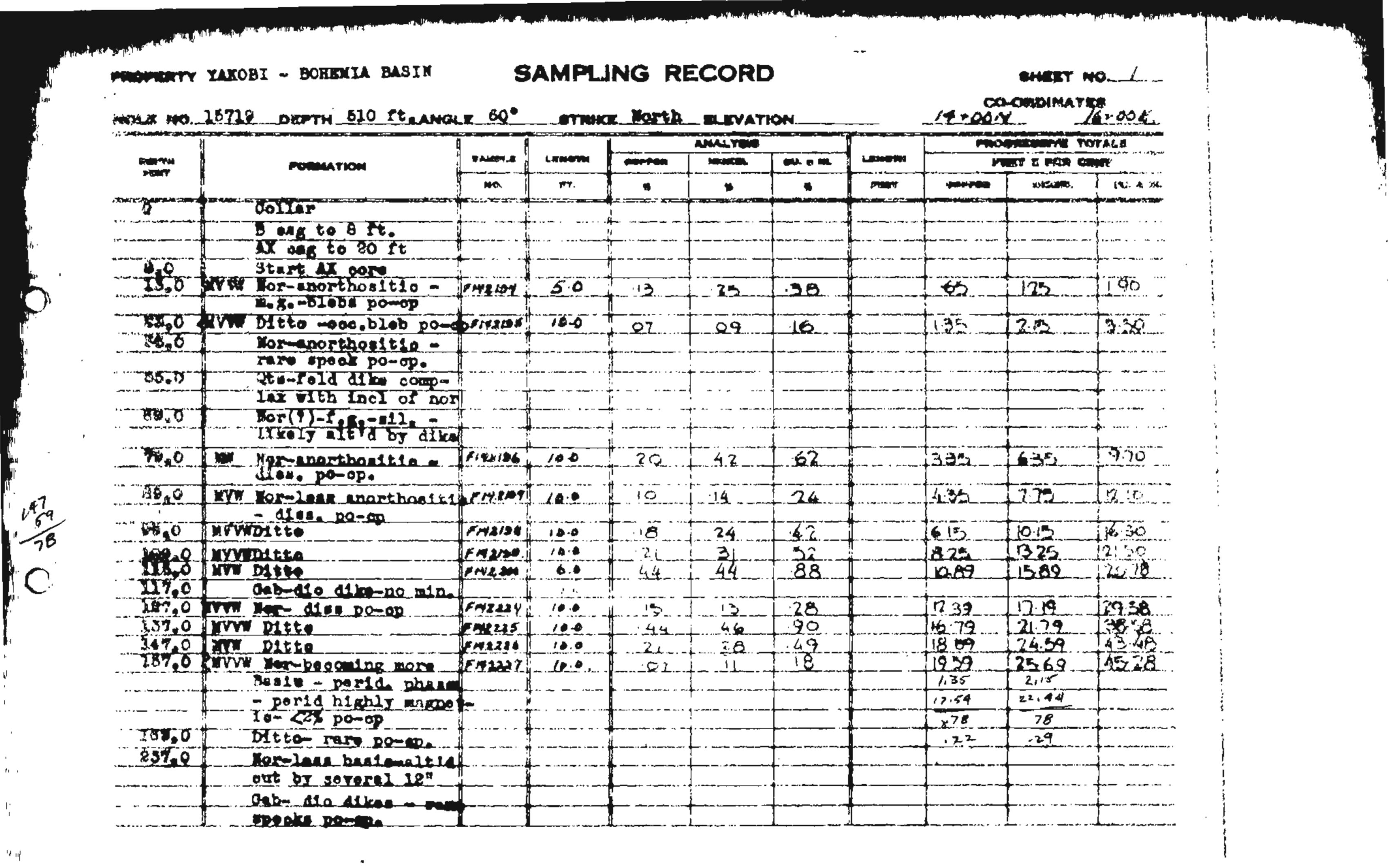


wour No. Lente

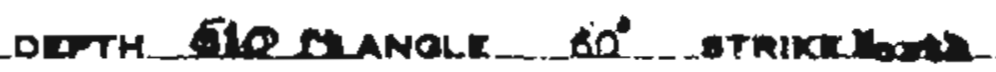

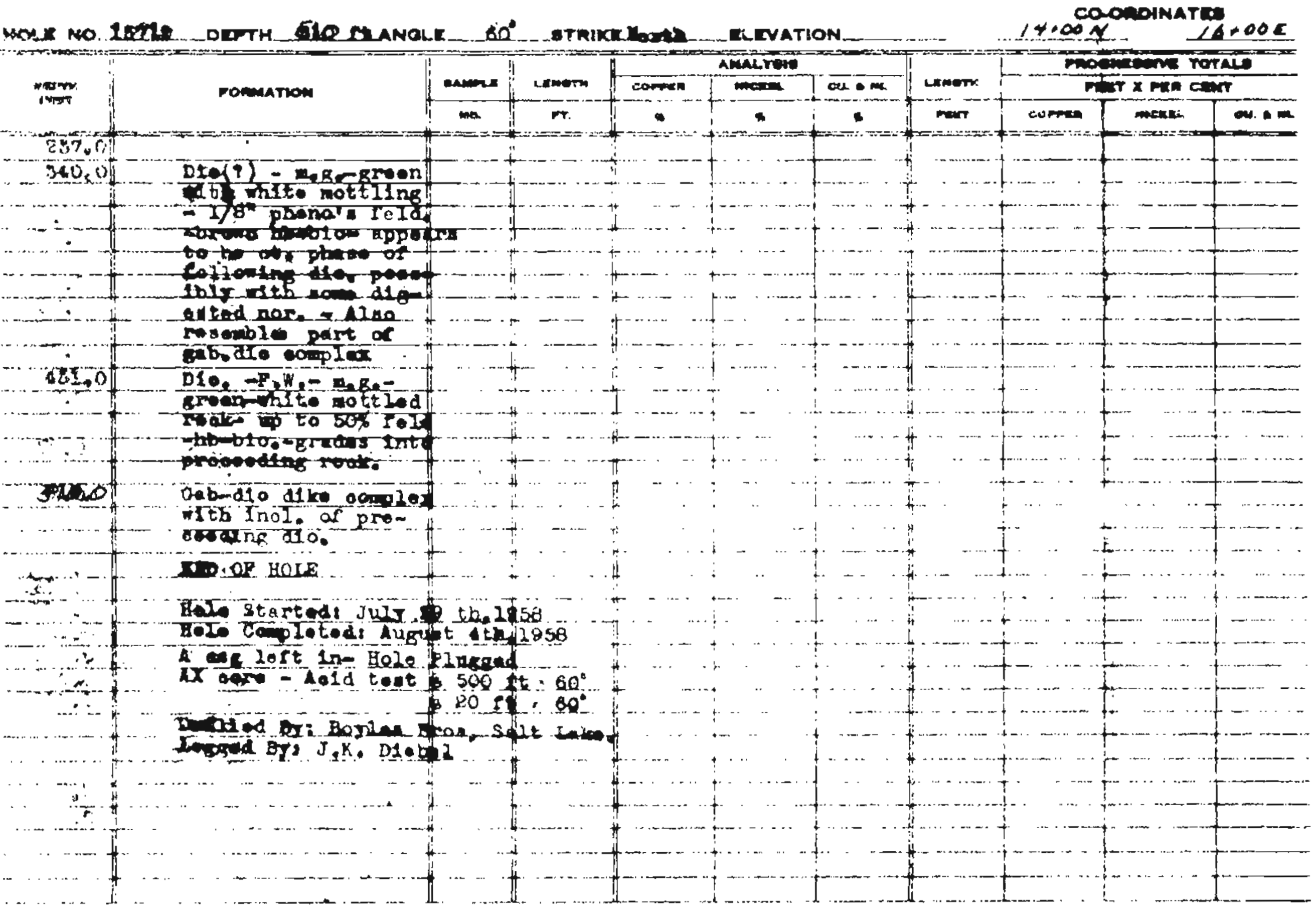




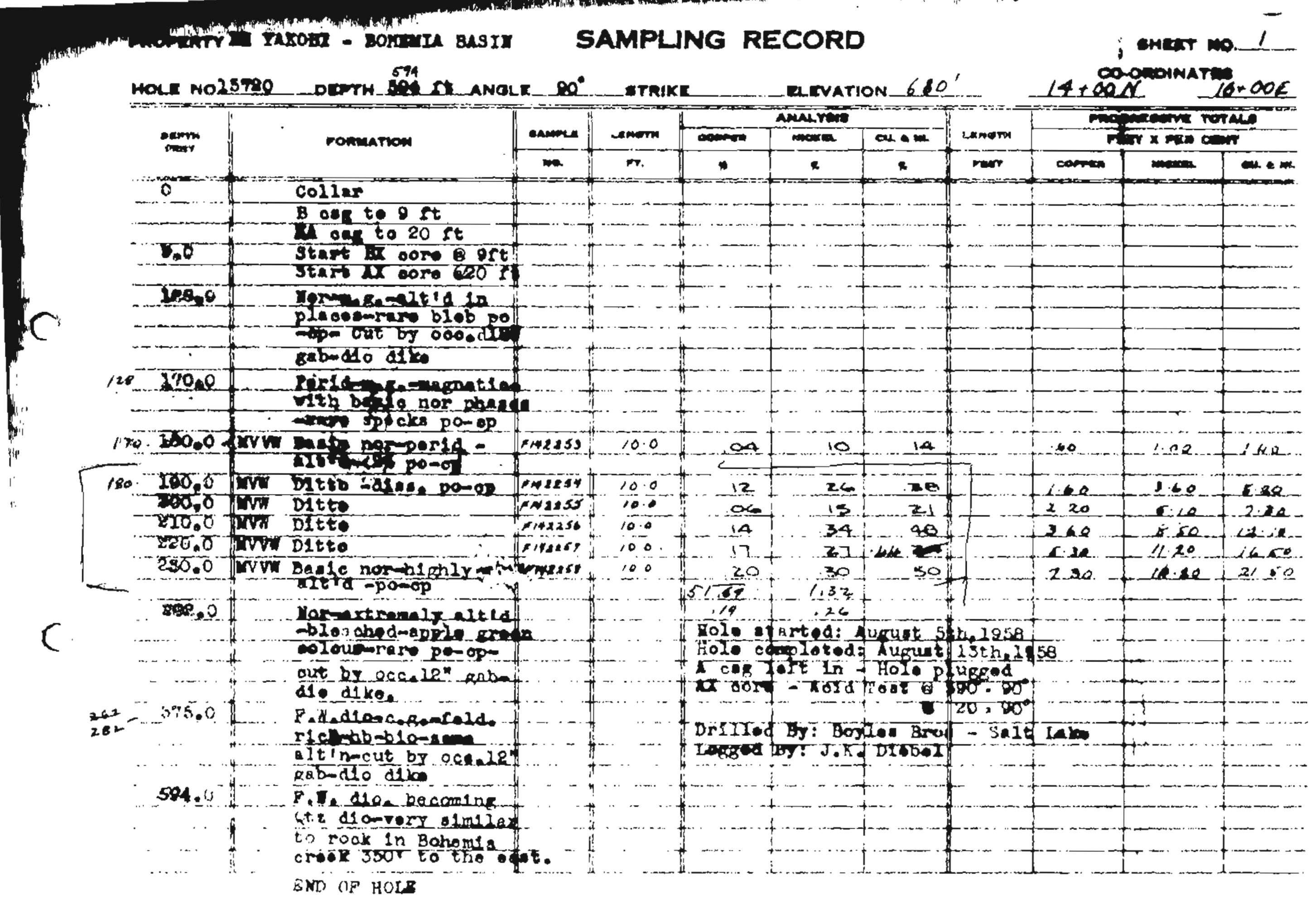




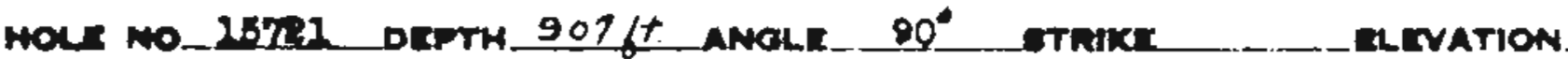
co-0molnatia

\begin{tabular}{|c|c|c|c|c|c|c|c|c|c|c|}
\hline orr no & 15721 DTrTH $9076 t$ ANG & 80 & & & & & & 1200 & S & gof \\
\hline & & & & & MaLrite & & & $=$ & andint & Taso \\
\hline m & Poneva tion & ensmer & S & coonenim & meres & $\infty A m$ & STmm & & $x \times \sin c$ & \\
\hline & & ma. & $\boldsymbol{\omega r}$ & $=$ & 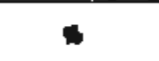 & $*$ & mart & $\cos$ & 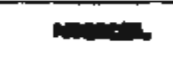 & -1.2 \\
\hline 0 & $\operatorname{col} 11 \pi$ & & & & & & & & & \\
\hline 10,0 & $\bar{B} a R-$ stant $\mathrm{BX}$ corn & & & & & & & & & \\
\hline & $010.0 \mathrm{rt}$ & & & & & & & & & 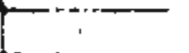 \\
\hline 20,0 & 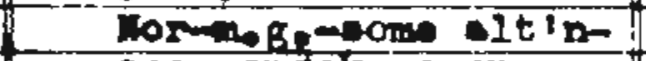 & & & & & & & & & \\
\hline & ooce sprole porcp. & & & & & & & & & \\
\hline & wed $\alpha$ og stare $d x$ & & & & & & & & & \\
\hline & 20126 & & & & & & & & & \\
\hline 640 & Tomede-c.ge- becanal & & & & & & & & & \\
\hline & Ins nor bälo Eoñra & & & & & & & & & \\
\hline & $641-500$ alt $n=$ & & & & & & & & & \\
\hline & 000, spadk po-op & & & & & & & & & \\
\hline 620 & 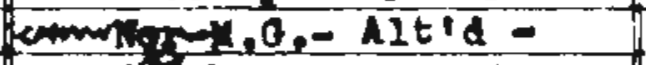 & {$[x+41 \sigma$} & 5.0 & 03 & 04 & $\mathrm{O}_{-}^{-}$ & & 13 & 20 & 35 \\
\hline & स $190 \mathrm{p}$ & & & & & & & & & \\
\hline 79.0 & WVI D1t5o-d1sspo- op & $617 \pi 159$ & 10 & 1,1 & $\therefore 12$ & 23 & & 175 & 1.40 & 2.65 \\
\hline 89.0 & 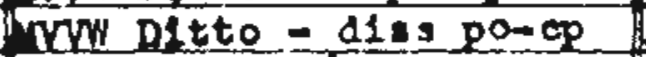 & $F / 42, s \psi$ & 10.0 & 11 & .19 & 30 & & 235 & 33 & 565 \\
\hline 92.0 & 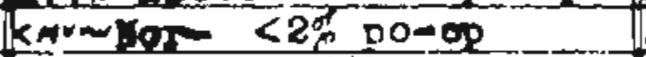 & $-1,2,59$ & 20 & 09 & .13 & 22 & & 253 & 3.56 & $6 \varnothing 9$ \\
\hline 92,0 & Gabodio atro & & & & & & & $-4+5$ & 20 & \\
\hline 03,0 & $70 x-a t^{+} d$ & & & & & & & 2.36 & 2,3 & \\
\hline 95,6 & Feld. Toln & & & & & & & 24 & & 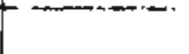 \\
\hline 145,0 & Ior-hIgiy altd in & & & & & & & 0.16 & 16 & \\
\hline & pIroet oet rpods po: & & & & & & & & & \\
\hline 156,0 & WunNorale'a dise po=op & $7 / 42156$ & 10.0 & 13 & 16 & 29 & $\gamma$ & 3830 & 5.16 & 3.90 \\
\hline 165.0 & Wh Nox-mor anosthoseldo & Errtsist & $\angle 0 \cdot 0^{\circ}$ & 30 & 46 & 76 & & 6830 & 976 & $10: 9$ \\
\hline & 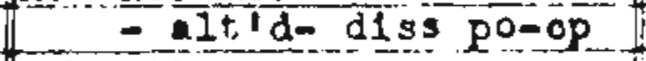 & & & & & & & & & \\
\hline 276,0 & NVW DItto & {$[1,4 \pi / 50$} & 100 & 28 & 46 & 74 & & 9630 & $\sqrt{436}$ & 2399 \\
\hline 185.0 & Irol $01 t t o$ & (x) $x, s+$ & 100 & 27 & -36 & -63 & & 12.33 & $\pi .96$ & 30.29 \\
\hline 195.0 & MWW Ditte & $51+2100$ & 100 & 16. & 25 & 41 & & 1393 & 20.46 & 3439 \\
\hline 205.0 & HW DIEEO InoIUdeA $12^{\prime \prime}$ & 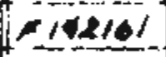 & 100 & 16 & 31 & 47 & & 1553 & 36 & 3909 \\
\hline & indorite diko & & & & & & & & & \\
\hline $8 \mathbf{1 8}$ & IV Dette includic $6 "$ & $F+16 \pm$ & 100 & 36 & 60 & 96 & & 19.12 & 2938 & 18.69 \\
\hline & rolda veln & & & & & & & & & \\
\hline 296.0 & Hor Hoxblahle natid & $(x+149$ & 10.0 & 16 & 26 & .42 & & 3073 & 32.16 & 52.89 \\
\hline & disspe-epo & & & +6 & 2.70 & & & & & \\
\hline
\end{tabular}




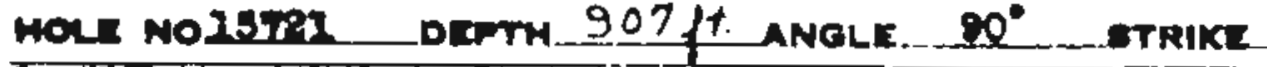
$1200 \%$

AMALrite

romation

\begin{tabular}{|c|c|c|c|c|}
\hline & & \multicolumn{3}{|c|}{ AMALYUES } \\
\hline ar & Lermor & $\cos t=$ & mextent & an $=m$ \\
\hline & $\boldsymbol{T}$. & m & . & • \\
\hline
\end{tabular}

225,0

P7. 0

De 0 es dibo

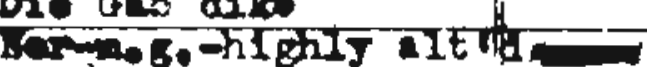

in oldote bes rut

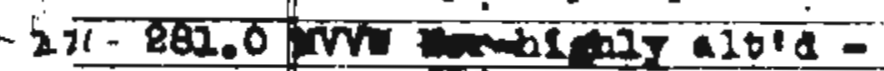

\section{Ses po-os}

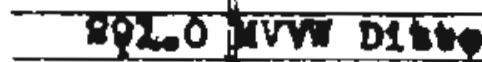

301.0 Krm Daste nor 24 geneo

(1)

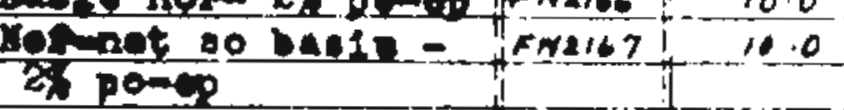

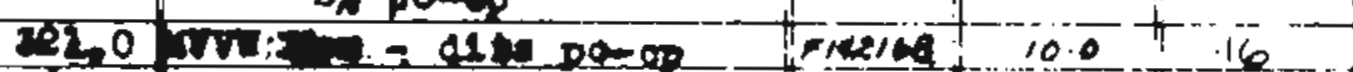

$21=328.0$ karratots..r<20 180.

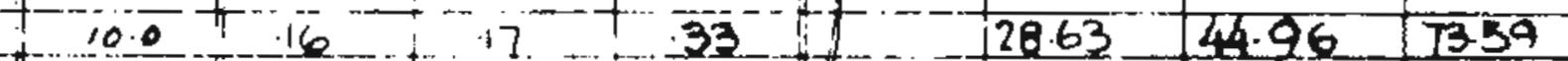

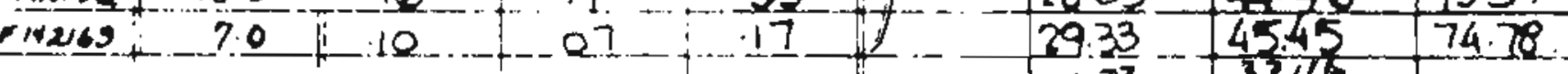

- of dike vating rrof

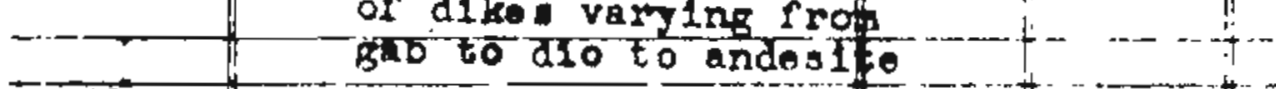

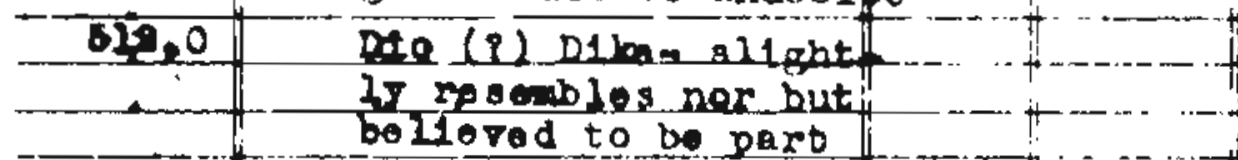

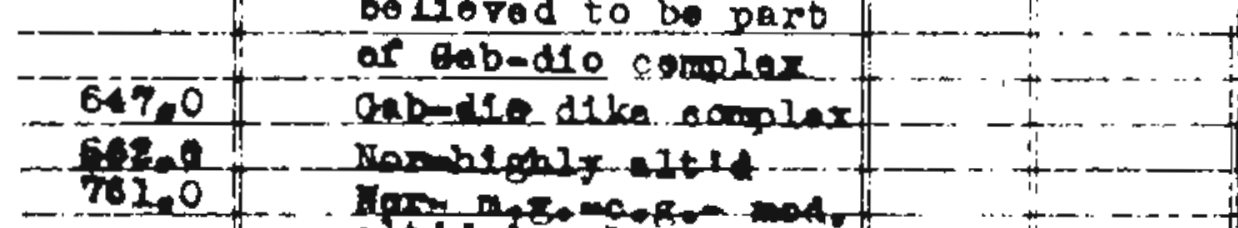

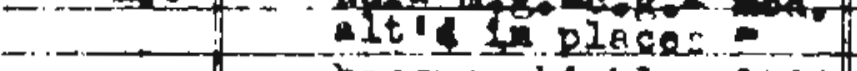

- Docones h1ghli alt!

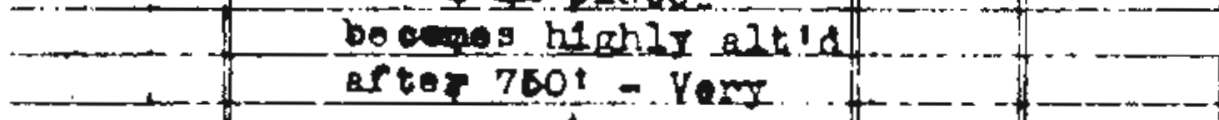

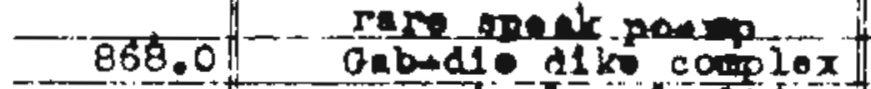

- oc. Incl. of sitid

507.0 -

oxt bT ooo, $12^{\prime \prime}$ geb-1

pomp a iong bogtstroak

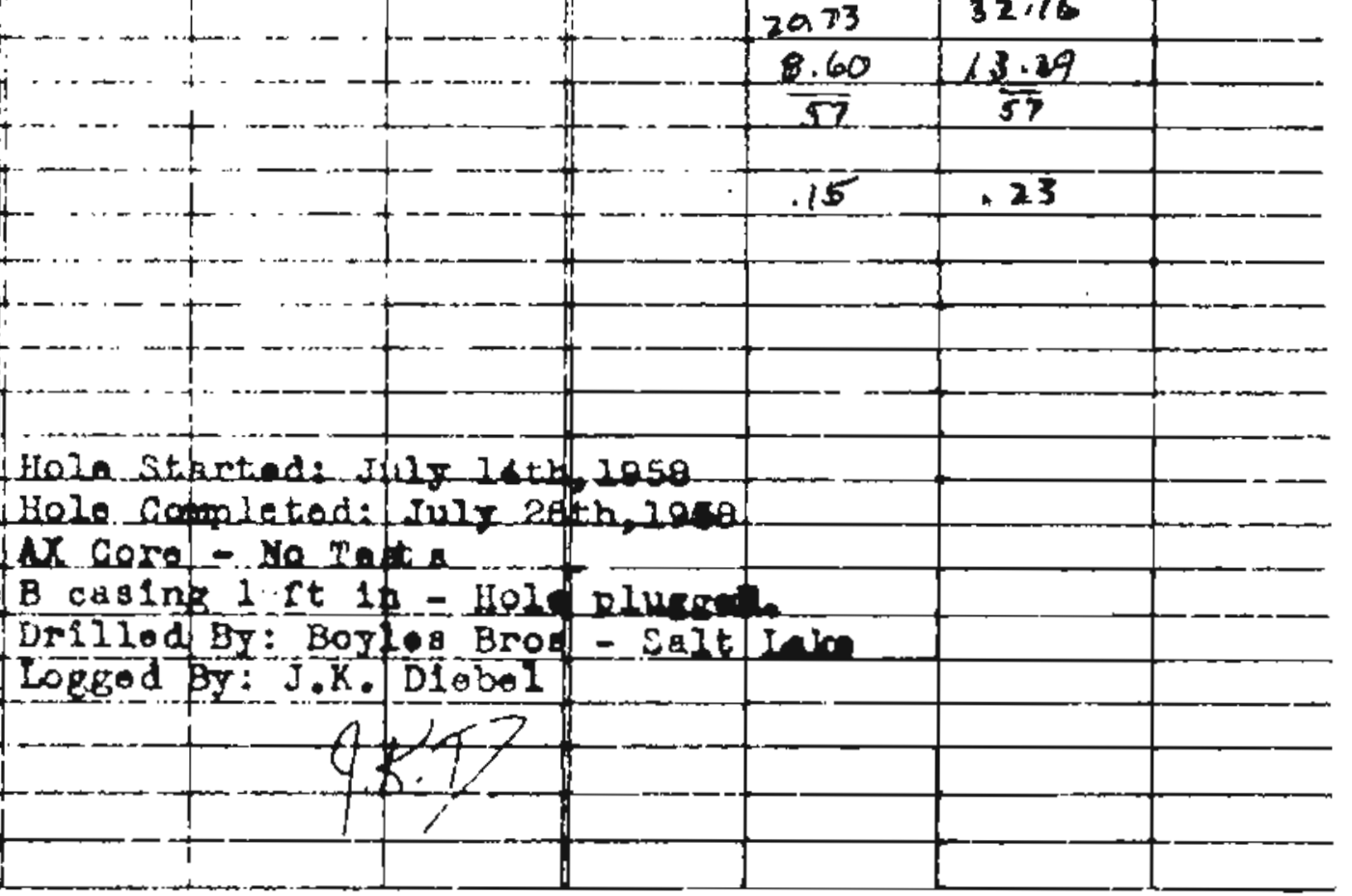




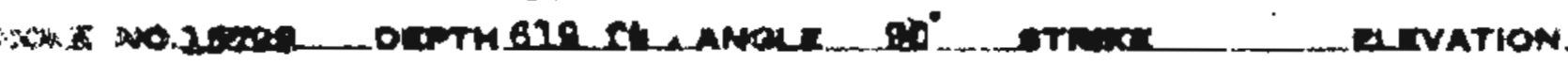




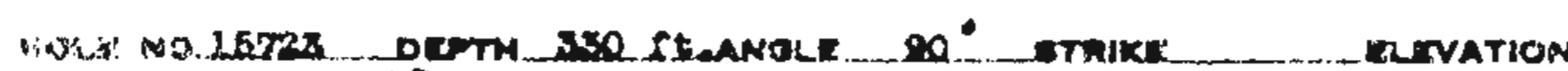
co-4woratro

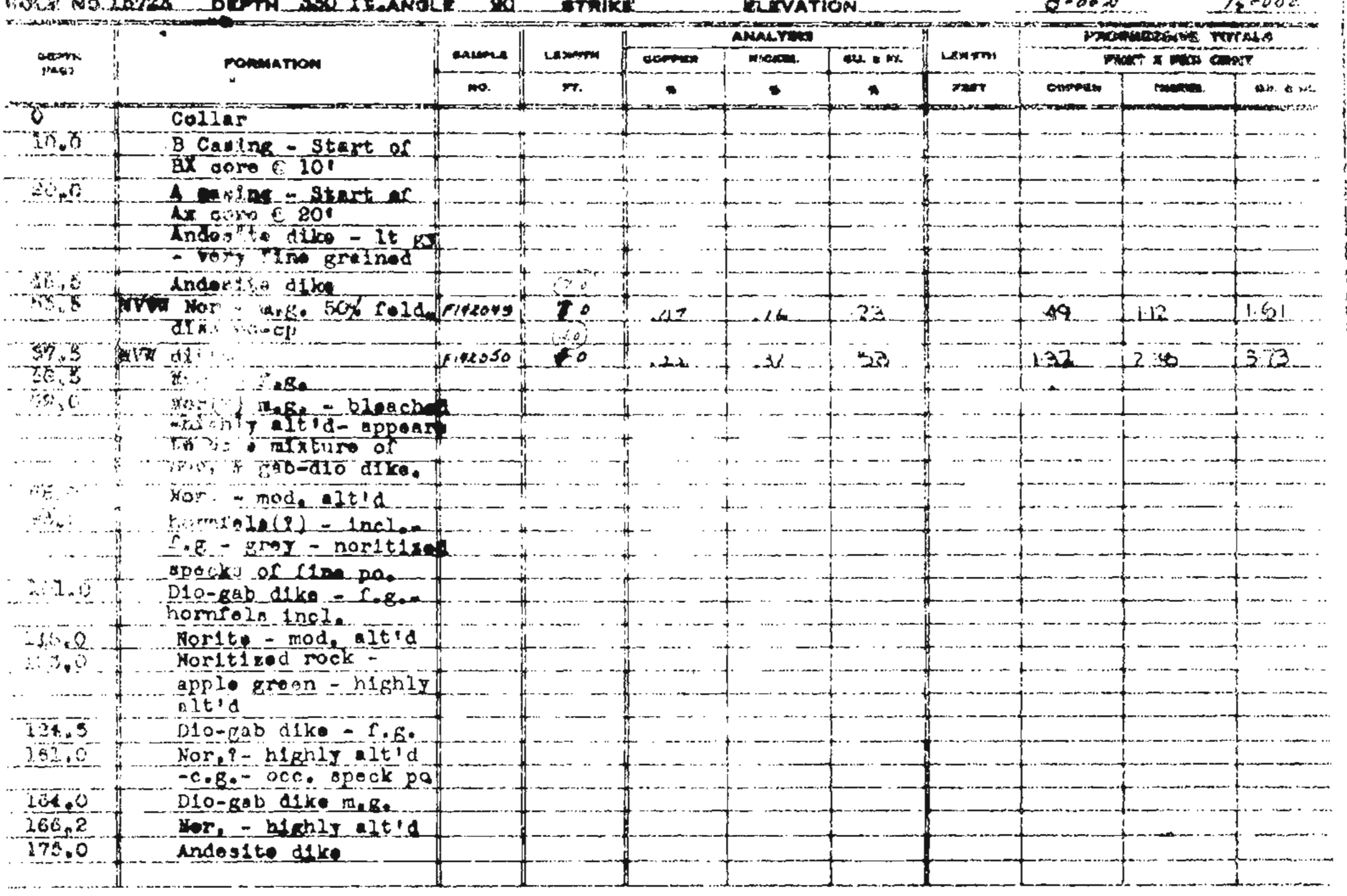




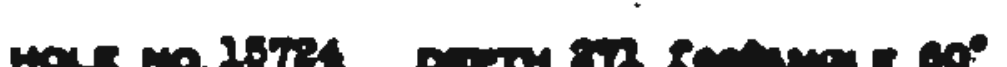

Mino. Me. Cunation 1550

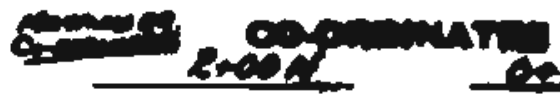

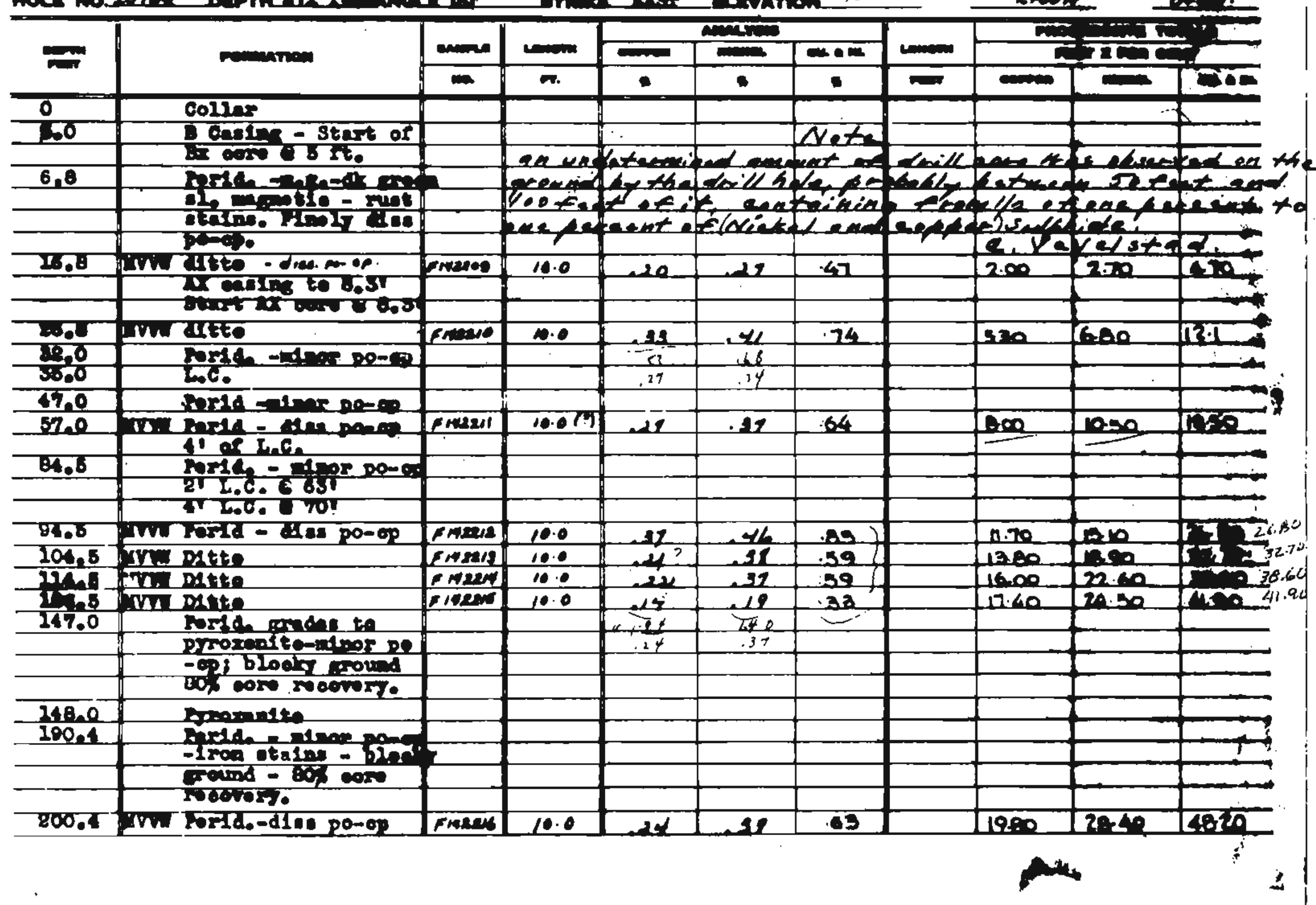




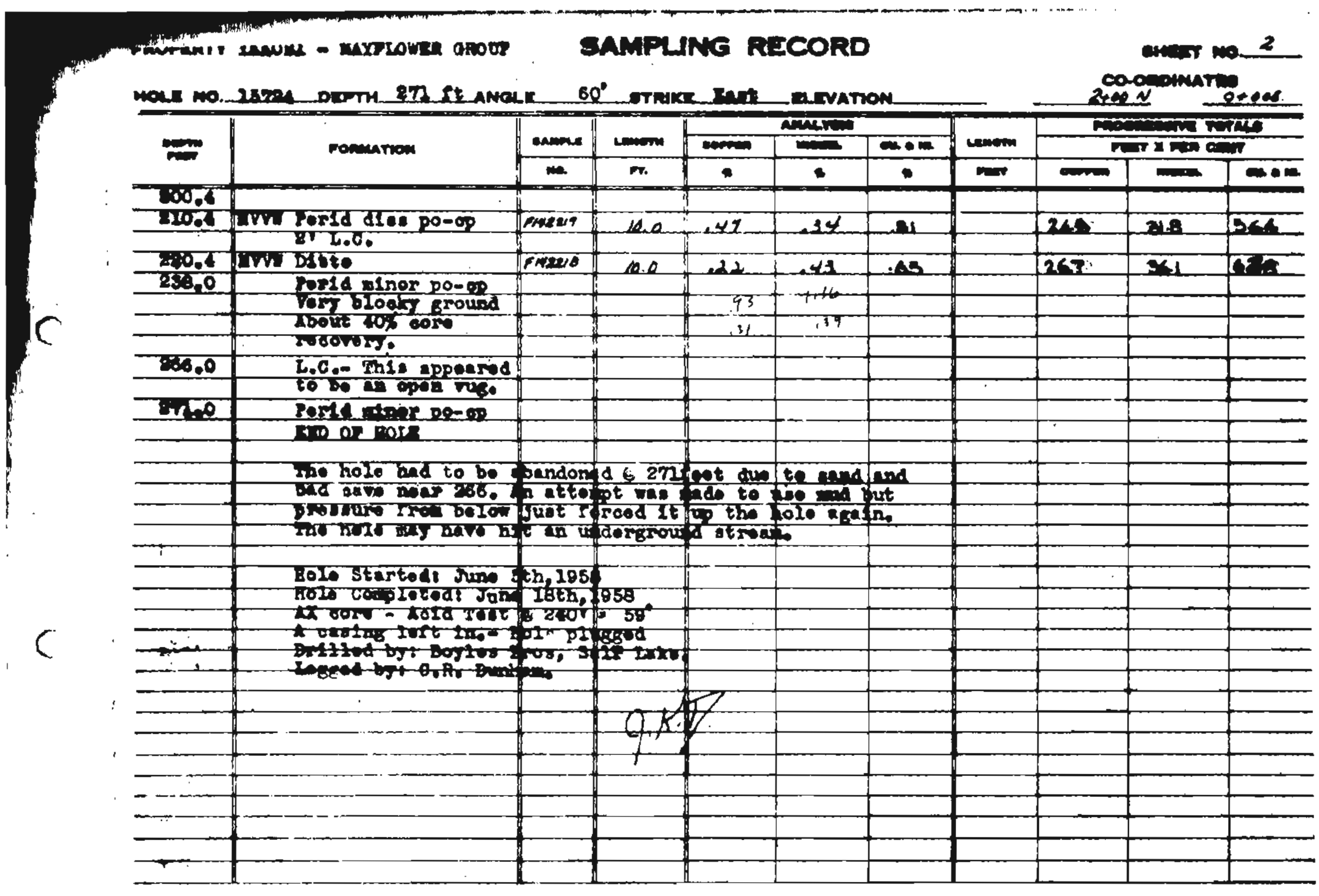


Mour Mo.15795 DEFTHEO ft ANCLE 60

etraxe lant arvation 1300 .

\begin{tabular}{|c|c|c|c|c|c|c|c|}
\hline & & & & & exee & & $1+12 x$ \\
\hline \multirow[b]{2}{*}{ Lemom } & \multicolumn{3}{|c|}{ Amaros } & \multirow[b]{2}{*}{ camom } & \multirow{2}{*}{\multicolumn{3}{|c|}{ miseming }} \\
\hline & \multirow{2}{*}{$\frac{00010}{9}$} & \multirow{2}{*}{$\frac{m}{6}$} & \multirow{2}{*}{$\frac{6+m}{1}$} & & & & \\
\hline$m$. & & & & $m$ & $\cos$ & $\operatorname{man}$ & $\frac{5}{a+a}$ \\
\hline & & & & & & & \\
\hline & & & & & & & \\
\hline & & & & & & & \\
\hline & & & & & & & \\
\hline & & & & & & & \\
\hline & & & & & & & \\
\hline & & & & & & & \\
\hline & & & & . & & & \\
\hline & & . & & & & & \\
\hline & & & & & & & \\
\hline & & & & & & & \\
\hline 10.0 & 26 & .44 & .69 & & 2.6 .0 & 4.90 & 625 \\
\hline & & & & & & & \\
\hline 10.0 & .12 & 20 & 32 & & 300 & 6.20 & 1010 \\
\hline & 31 & E & & & & & \\
\hline & .12 & 13 & & & & & \\
\hline & & & & & & & \\
\hline & & & & & & & \\
\hline & & & & & & & \\
\hline 10.0 & .19 & $\ldots 1$ & 52 & & 570 & 9.60 & 18 \\
\hline & & & & & & & \\
\hline 10.0 & .08 & 25 & 34 & & 160 & 12.10 & 137 \\
\hline & -121 & .51 & & & & & \\
\hline & -14 & $1: 1$ & & & & & \\
\hline & & & & & & & \\
\hline & & & & & & & \\
\hline & & & & & & & \\
\hline & & & & & & & \\
\hline & & & & & & & \\
\hline & & & & & & & \\
\hline & & & & & & & \\
\hline $10 \cdot 0$ & -14 & .29 & 43 & & 0.00 & 100 & 22.6 \\
\hline & & & & & & & \\
\hline
\end{tabular}


MOLE NO 25725 _.DEPTH 230 IE ANGLE 60

STRIKc Eart_-_Lrvation co-conowarts

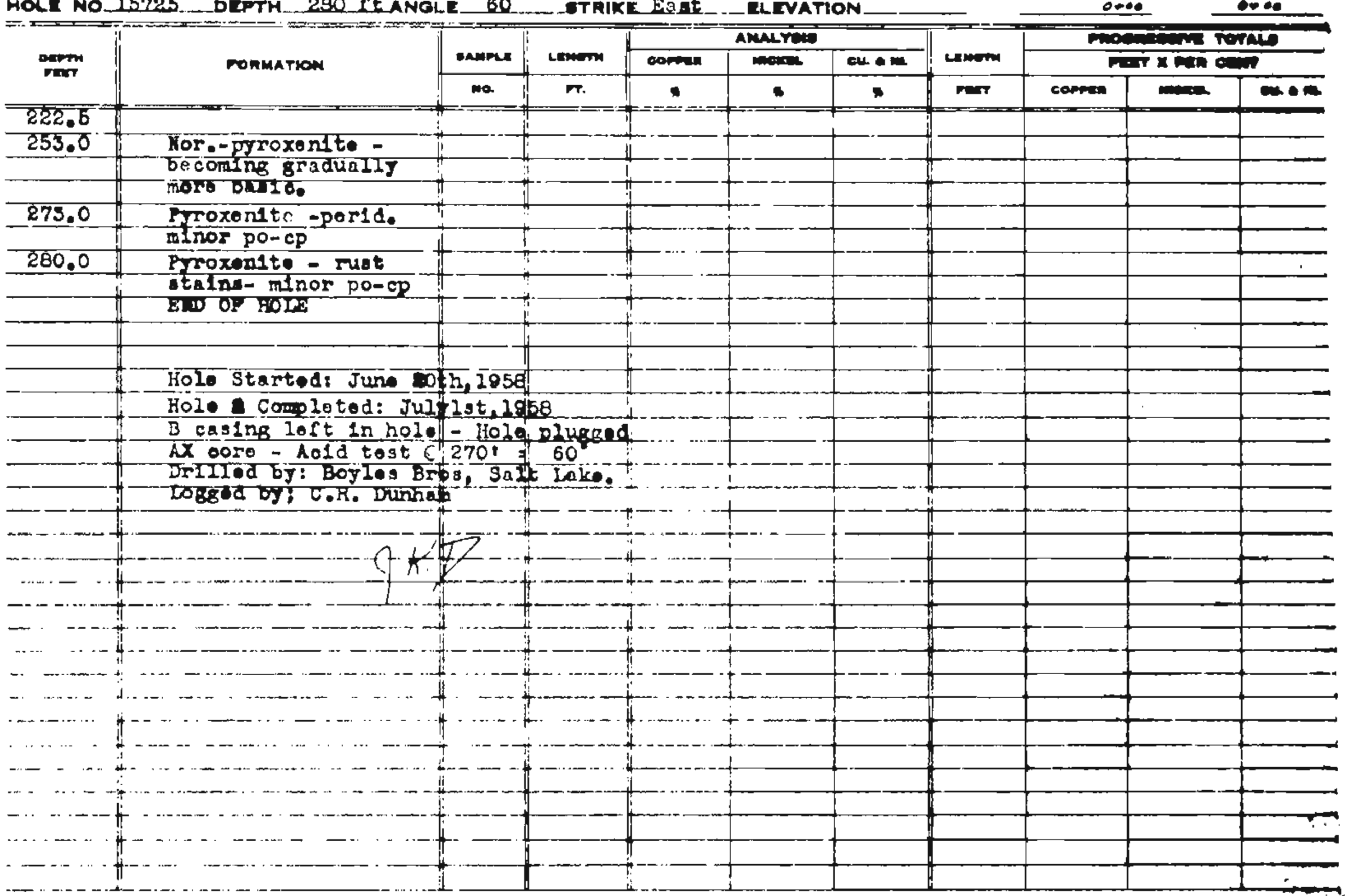




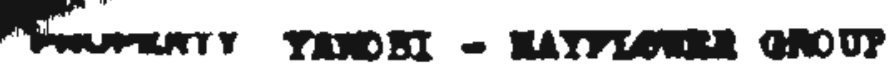

DAMMLUNG RECORD

$\cos 1$

mour mo.jerah orom

Anour a

ormace rate

\begin{tabular}{|c|c|c|c|c|c|c|c|c|c|c|}
\hline \multirow{3}{*}{$\operatorname{mom}$} & \multirow{3}{*}{ moninariom } & \multirow{3}{*}{ a } & \multirow{3}{*}{ Lemem } & \multicolumn{3}{|c|}{ Avarros } & \multirow{3}{*}{$\frac{\operatorname{Ln} m}{m}$} & \multirow{2}{*}{\multicolumn{3}{|c|}{$\vec{n}, \mathrm{~min}$}} \\
\hline & & & & $\operatorname{man}$ & $\operatorname{mon}$ & n. & & & & \\
\hline & & & & - & - & - & & comom & $m$ & 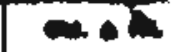 \\
\hline \multirow{2}{*}{\multicolumn{11}{|c|}{$\begin{array}{l}\text { Wollh } \\
\text { B. Cope Shart Bx }\end{array}$}} \\
\hline & & & & & & & & & & \\
\hline & 302210 & & & & & & & & & \\
\hline \multicolumn{11}{|c|}{ Doranorthon1t10- } \\
\hline & 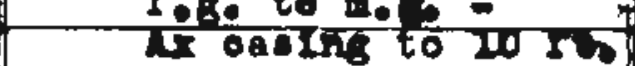 & & & & & & & & & \\
\hline \multirow{3}{*}{80.0} & secte $0 \times 8020$ e 100 & & & & & & & & & \\
\hline & 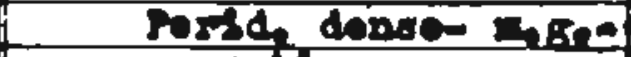 & & & & & & & & & \\
\hline & $\operatorname{magnot10}=\operatorname{sax}$ & & & & & & & & & \\
\hline 50.0 & pitfo - dine po-ch & $\operatorname{lng} 190$ & 10.0 & 10 & 22 & 32 & & $1 . \infty$ & 720 & 3.20 \\
\hline 10,0 & WVT Perid- laole lor - & ganitin & 11.0 & .22 & 20 & 32 & & 2.20 & 420 & $6 \sqrt{0}$ \\
\hline & nemedu arse po- & & & & & & & & & \\
\hline & W. & & & & & & & & & \\
\hline 50,0 & WV Deto- wory bloag & $\sin 2$ & 10.0 & 07 & 15 & 27. & -1 & 290 & 370 & 60 \\
\hline 60.0 & ont porid-Bedig Det & nimitg & $70.0^{-}-1+1-10$ & 89 & 15 & 2.4 & & 380 & 720 & $11 . \infty$ \\
\hline & pant10-kejpo- & & & & & & & & & \\
\hline 70.0 & Aw Perte-Bale nor = & FA\&III & 160 & -16 & 22 & 38 & & 3.40 & 940 & 16.20 \\
\hline $8 \% 0$ & 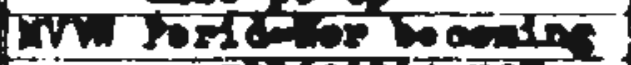 & $E\left[\frac{19}{2}\right.$ & 10. & il & .15 & 26 & & 650 & 0.90 & $\pi .40$ \\
\hline & 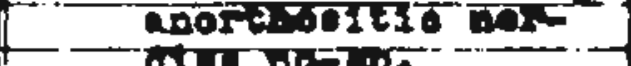 & & & & 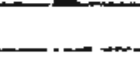 & & & & & \\
\hline 80. & 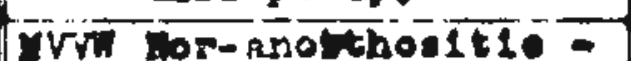 & ranems & 10 & $\overline{14}$ & 19 & $3 \overline{3}$ & & 790 & $\frac{\pi}{2}$ & 2070 \\
\hline & 600 nng bango & & & & & & & & & \\
\hline 100.0 & $=\operatorname{ding} p a-c$ & & & & & & & & & \\
\hline 20.0 & 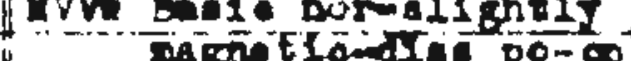 & & & Al. & -18 & -29 & & 900 & 1460 & $13 \mathbf{2}^{\circ}$ \\
\hline$\pi v .0$ & 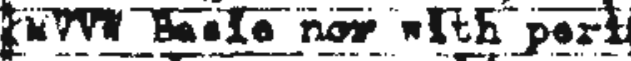 & tanend & 10.0 & 09 & 19 & 28 & & 290 & 1450 & 2668 \\
\hline & $\cos ^{\mathrm{s} a}=$ & & & & & & & & & \\
\hline & Re bet & $\operatorname{anen}$ & 10.6 &.$\overline{9}$ & 5 & 24 & & 1080 & 1800 & 7.80 \\
\hline $2 x$ & Trut Dite & mances & $\infty .0$ & og & 14 & 23 & & 1170 & 1940 & $3+10^{2}$ \\
\hline
\end{tabular}


ohet mo. 2

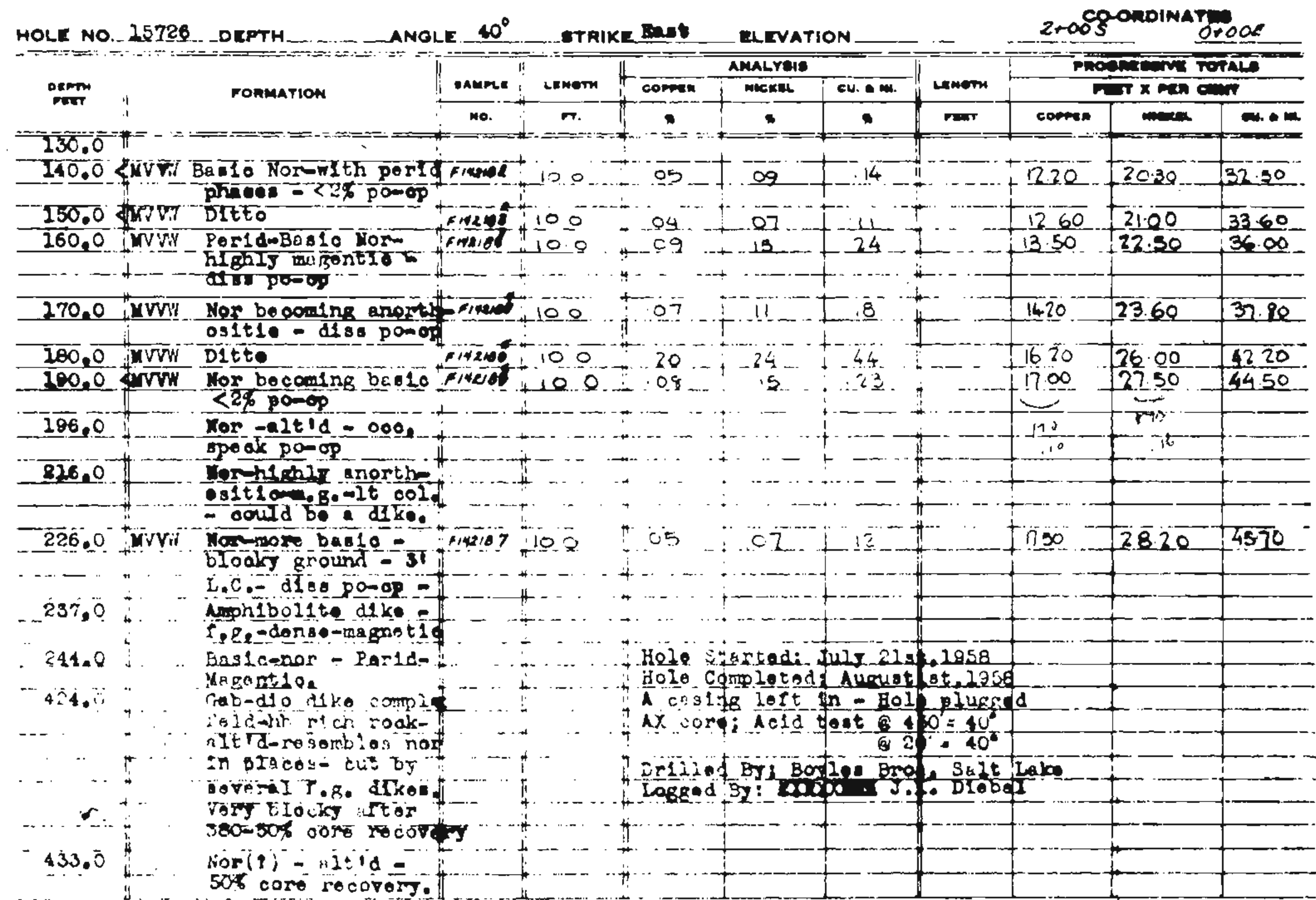
END OF HOIE 


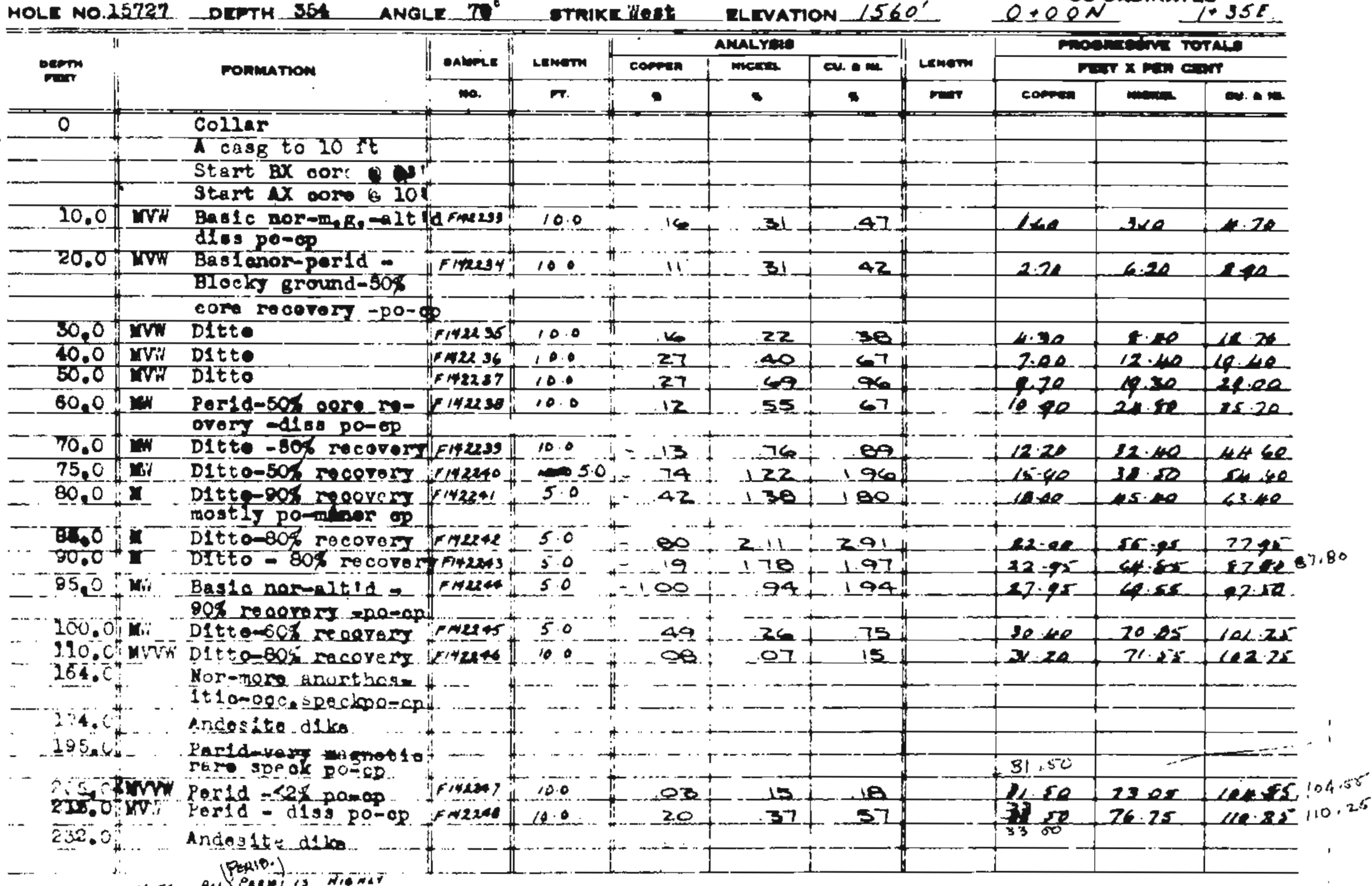

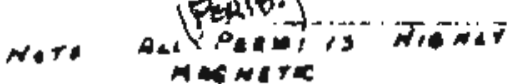


_otaike Wot _ ELEVATION_ $1560^{\prime}$ $\operatorname{lnm}$

FonMation

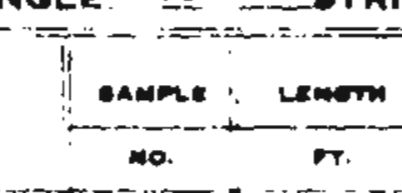

\begin{tabular}{|c|c|c|}
\hline & ANALTUE & \\
\hline connin & wieren & eu. $1 m$ \\
\hline 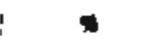 & 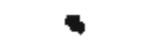 & - \\
\hline
\end{tabular}

OHET NO.-는...

332.0 mo.

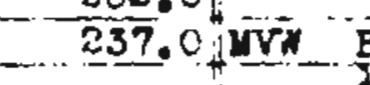

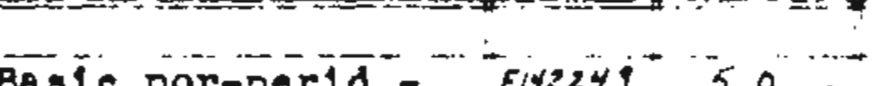

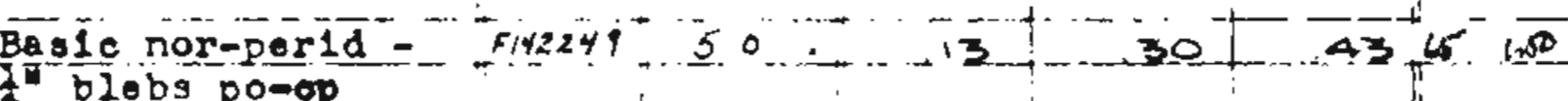

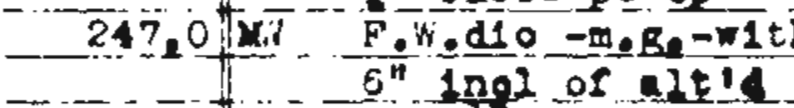
250.0i

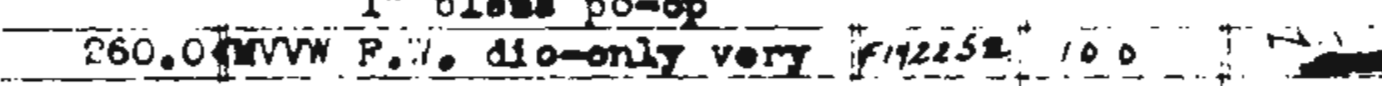

- oec upk pose

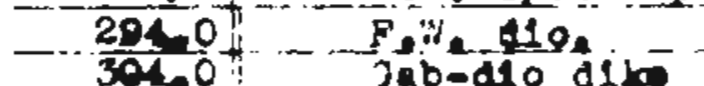

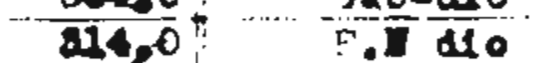

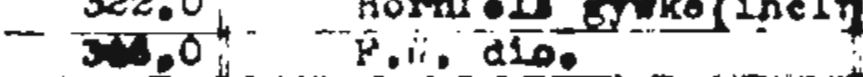

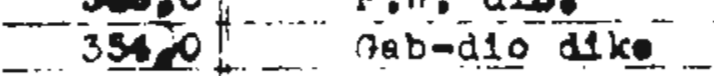

rino oP HOIE

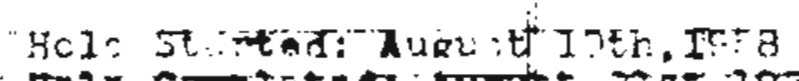

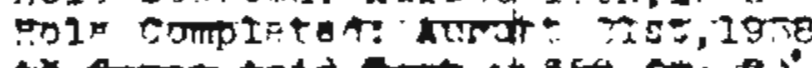

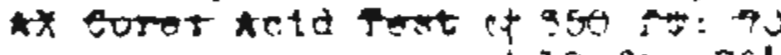

$A \operatorname{osph}$ zort in

स $190+790$

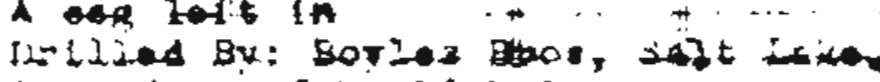

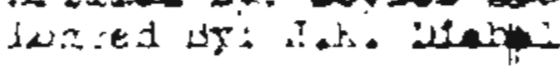

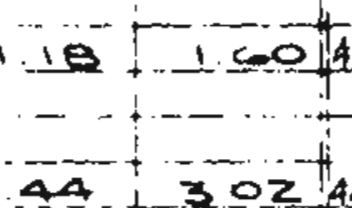

itaon
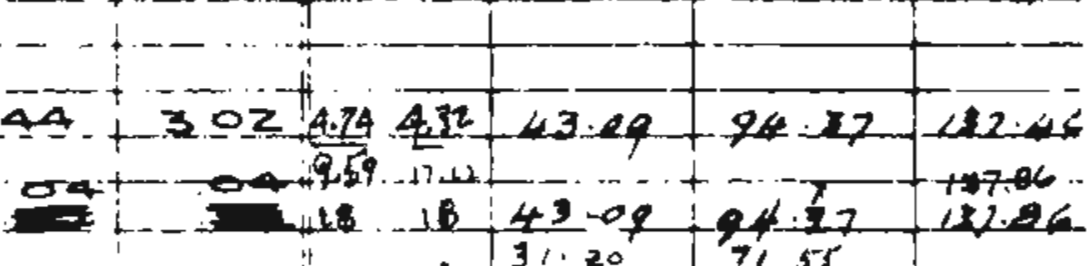
QN $\quad 1+356$ The retal

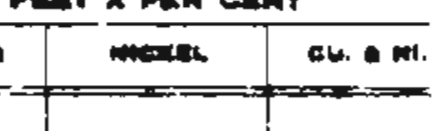

2125.142 .42 
eHath Mo. I

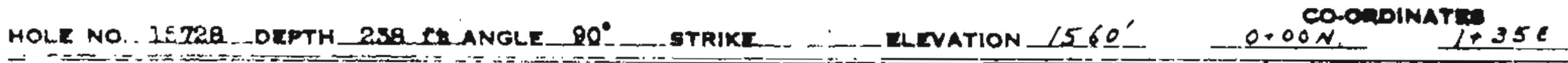
A oxmm

ronmation

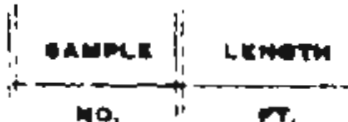

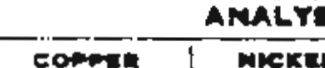

min x men coint

$-\frac{-1}{0}=-\cdots$

Coligs

AX cog to 2 st

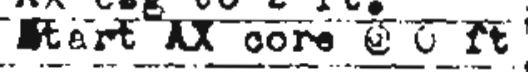

Basio norme

fizezs 100

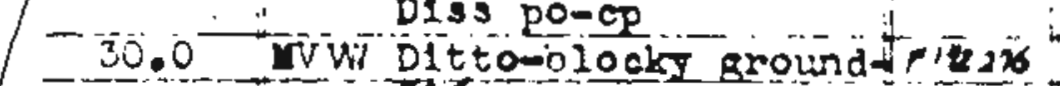

$50 \%$ core rocovery

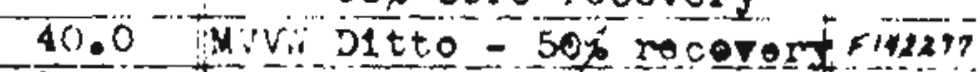

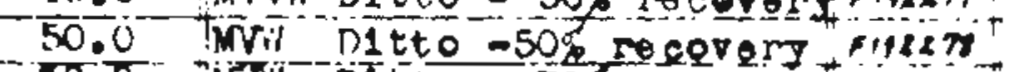

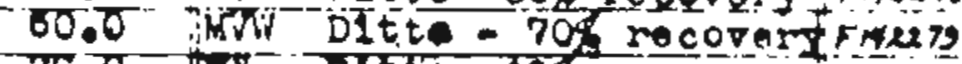

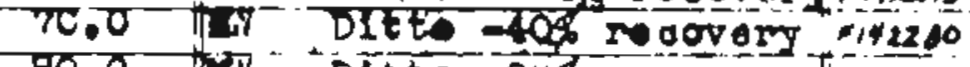

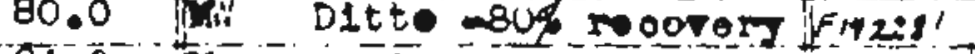

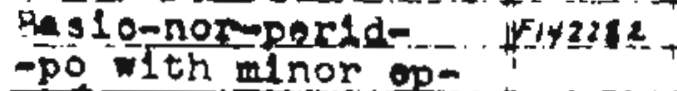

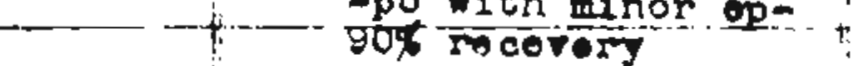

I00.0

120.0 Mvi

Diteo =70\% necovar fitiss

20,0

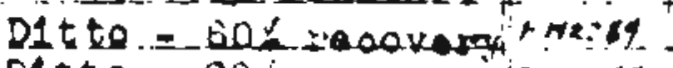

Ditto-90 moyaryle cuats

Bes1c nor -paide.

$E$

altd in places = sef.

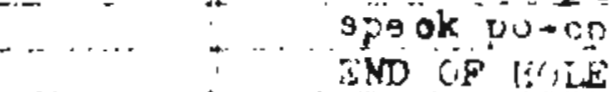

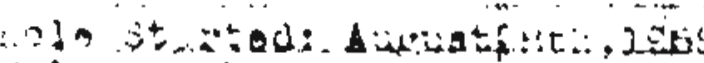

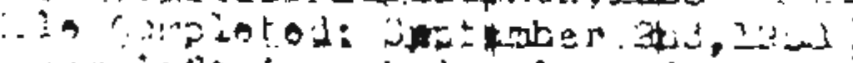

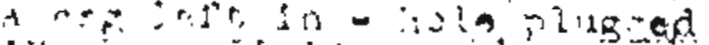

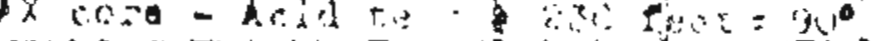

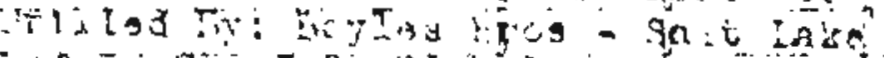

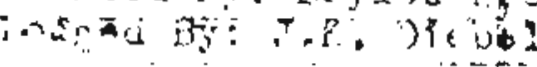

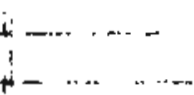

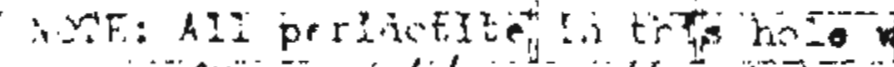

"All" pendotite on this hold is highty wagrafie. 


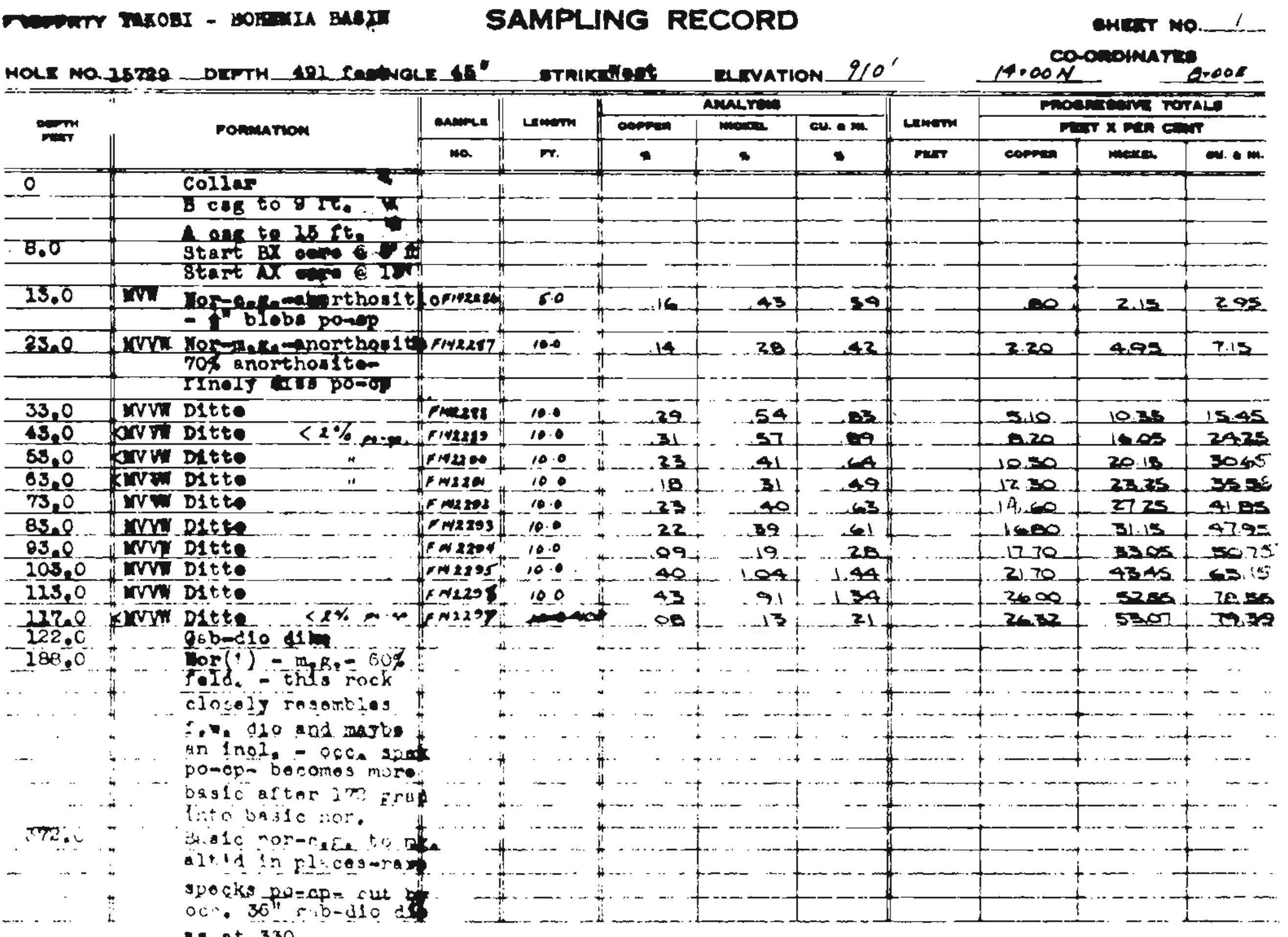




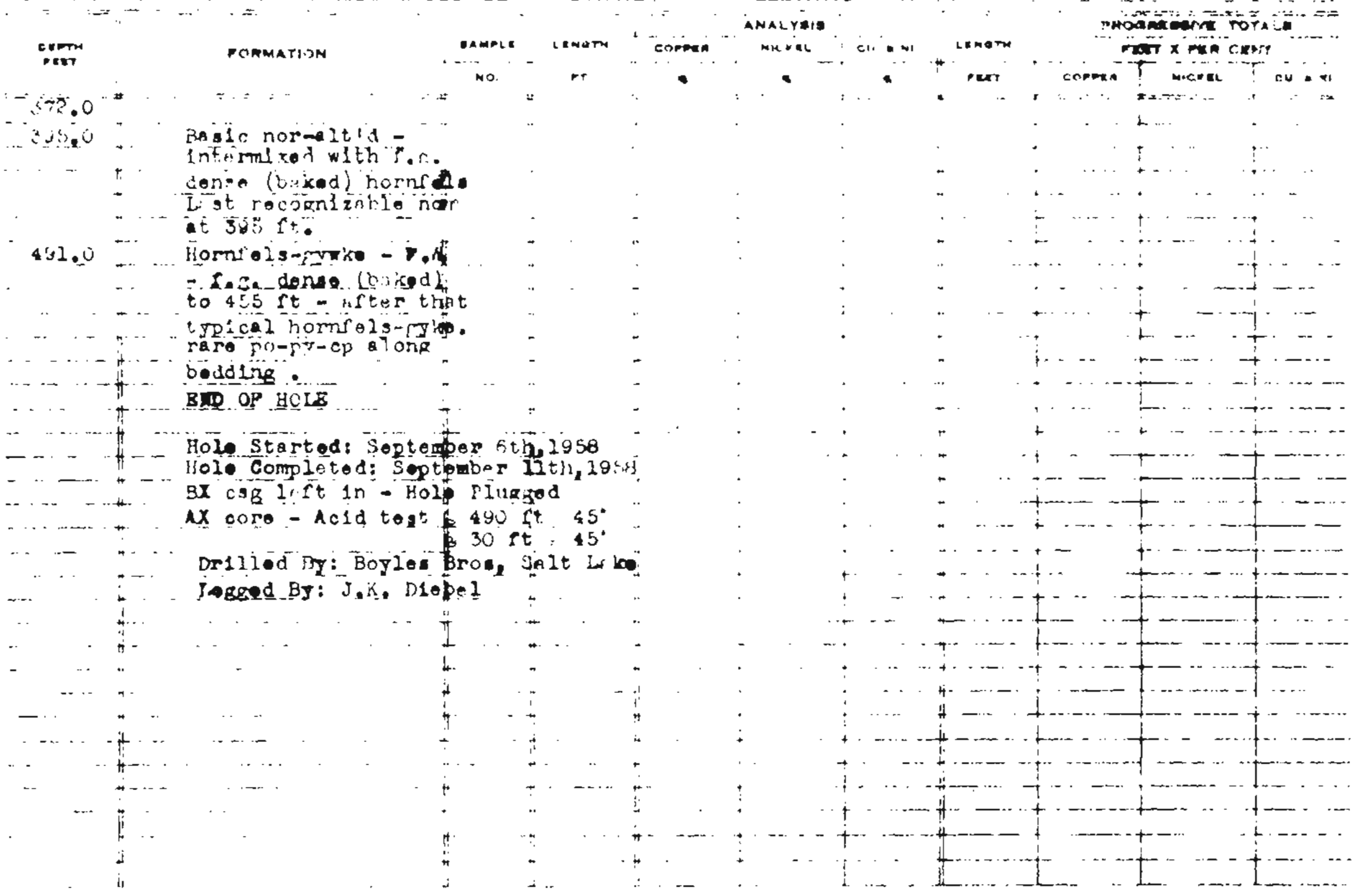

